Florida International University

FIU Digital Commons

6-21-2019

\title{
Boron Nitride Nanotube Reinforced Titanium Composite with Controlled Interfacial Reactions by Spark Plasma Sintering
}

Jenniffer Bustillos

Florida International University, jbust035@fiu.edu

Follow this and additional works at: https://digitalcommons.fiu.edu/etd

Part of the Materials Science and Engineering Commons

\section{Recommended Citation}

Bustillos, Jenniffer, "Boron Nitride Nanotube Reinforced Titanium Composite with Controlled Interfacial Reactions by Spark Plasma Sintering" (2019). FIU Electronic Theses and Dissertations. 4241.

https://digitalcommons.fiu.edu/etd/4241

This work is brought to you for free and open access by the University Graduate School at FIU Digital Commons. It has been accepted for inclusion in FIU Electronic Theses and Dissertations by an authorized administrator of FIU Digital Commons. For more information, please contact dcc@fiu.edu. 


\title{
FLORIDA INTERNATIONAL UNIVERSITY
}

Miami, Florida

\begin{abstract}
BORON NITRIDE NANOTUBE REINFORCED TITANIUM COMPOSITE WITH CONTROLLED INTERFACIAL REACTIONS BY SPARK PLASMA SINTERING
\end{abstract}

A thesis submitted in partial fulfillment of

the requirements for the degree of

MASTER OF SCIENCE

in

MATERIALS SCIENCE AND ENGINEERING

by

Jenniffer Bustillos 


\section{To: Dean John Volakis}

College of Engineering and Computing

This thesis, written by Jenniffer Bustillos, and entitled Boron Nitride Nanotube Reinforced Titanium Composite with Controlled Interfacial Reactions by Spark Plasma Sintering, having been approved in respect to style and intellectual content, is referred to you for judgment.

We have read this thesis and recommend that it be approved.

\section{Zhe Cheng}

$\overline{\text { Benjamin Boesl, Co-Major Professor }}$

Arvind Agarwal, Co-Major Professor

Date of Defense: June 21, 2019

The thesis of Jenniffer Bustillos is approved.

Dean John Volakis College of Engineering and Computing

Andrés G. Gil

Vice President for Research and Economic Development and Dean of the University Graduate School

Florida International University, 2019 


\section{DEDICATION}

This thesis is dedicated to my always supportive aunts Rosa Bustillos-Wong and Gisella Bustillos-Wong. Your support throughout my life was the catalyst to the opportunities I have today and the many more to come. 


\section{ACKNOWLEDGMENTS}

I express my outmost sincere gratitude to my advisors, Prof. Arvind Agarwal and Prof. Benjamin Boesl, for the never-ending academic and professional advice provided throughout my undergraduate and graduate research career. I am grateful for the priceless opportunities you have given me to learn and grow as an academic in the field. Your mentorship has been vital to empower my future career as a graduate student and a future professional. I would also like to extend my gratitude to my committee member Prof. Zhe Cheng for the continuous advice and helpful suggestions provided. I would like to acknowledge the support from the ONR grant \# N00014-17-1-2563, and Dr. Roy Whitney of BNNT, LLC for the supply of nanotubes used in this study.

I am most grateful for the great team of students and alumni at the Plasma Forming Laboratory, who has been part of my journey as a researcher. Special thanks to Dr. Cheng Zhang, you have instigated in me the habit to question things scientifically; Ms. Ana Exime, your pursuit for new opportunities inspire me not to be afraid of the unknown; and Ms. Luiza Da Fontoura, your happiness even in moments of struggle motivate me to pursuit my dreams. Ms. Archana Loganathan, and Mr. Pranjal Nautiyal you have been continuous support throughout my academic career, serving as mentors and role models. Most importantly, I would like to thank my family, who have supported me in all endeavors. Most grateful to my parents who have provided unconditional love and support over the distance. My aunts, Rosa and Gisella for the enormous support and patience, without you none of this would be possible. My achievements and success are shared with you and a token of my appreciation. I would like to acknowledge my aunt Carola Bustillos, brother (Miguel) and sister (Gisella) and her growing family. 


\begin{abstract}
OF THE THESIS
BORON NITRIDE NANOTUBE REINFORCED TITANIUM COMPOSITE WITH

CONTROLLED INTERFACIAL REACTIONS BY SPARK PLASMA SINTERING
\end{abstract}

by

Jenniffer Bustillos

Florida International University, 2019

Miami, Florida

Professor, Arvind Agarwal, Co-Major Professor
Professor Benjamin Boesl, Co-Major Professor

In this study, Boron Nitride Nanotube (BNNT) reinforced Titanium matrix composites are synthesized by Spark Plasma Sintering. Two main challenges directly affecting the mechanical performance of BNNT-metal matrix composites are addressed:(i) Homogenous dispersion of high surface energy BNNTs, and (ii) Controlling interfacial reactions at the metal/nanotube interface. High affinity of acetone with BNNTs and high energy ultrasonication induced the mechanical dispersion and stability of BNNTs in their dispersion. The sintering of $\operatorname{Ti}$ (99\% relative density) was achieved at 50\% less processing temperature than those used in conventional sintering to minimize interfacial reactions when reinforced with BNNTs. The reduction of temperatures in addition to the reduction (by $91 \%$ ) in processing times was shown to control reaction phases. Bulk compressive yield strengths of Ti-BNNT sintered at low $\left(750^{\circ} \mathrm{C}\right)$ and high $\left(950^{\circ} \mathrm{C}\right)$ temperatures were improved by $21 \%$ and $50 \%$ respectively, as compared to Ti alloy without reinforcement. Twin boundaries, pinning of dislocations by BNNTs, and crack bridging were strengthening mechanisms identified in the composites. 


\section{TABLE OF CONTENT}

CHAPTER

PAGE

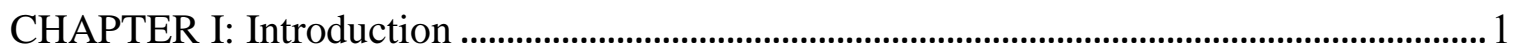

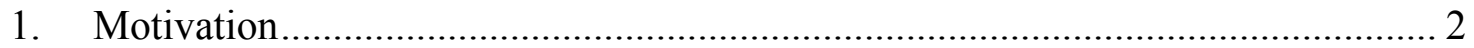

1.1 Demand for lightweight and high strength structural materials ......................... 2

1.2 Boron Nitride Nanotubes (BNNTs): Potential reinforcement of Ti-matrices .... 3

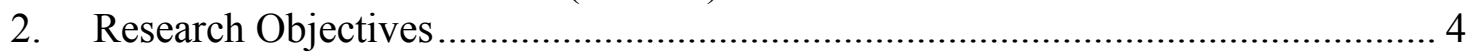

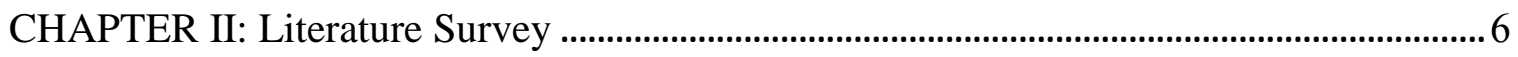

1. Boron Nitride Nanotubes (BNNTs): A mechanically, chemically, and thermally

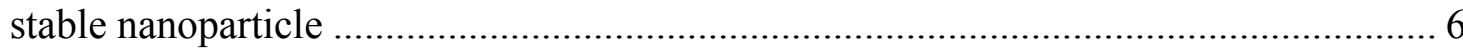

1.1 Physical, chemical and mechanical properties................................................ 7

1.2 Synthesis-dependent morphology of BNNTs: Bamboo vs. smooth ................... 8

1.3 Boron Nitride Nanotube-reinforced composites …………………………....... 13

1.3.1 BNNT reinforced polymer composites ................................................... 14

1.3.2 BNNT reinforced ceramic composites................................................. 17

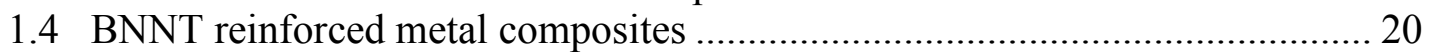

1.5 BNNT reinforced Titanium matrix composites ............................................ 28

1.5.1 Titanium: A suitable metal for extreme environments ………………...... 28

1.5.2 Integrating Boron Nanotubes in a Titanium Matrix ……………............. 32

1.5.3 Current challenges: .............................................................................. 36

CHAPTER III: MATERIALS \& EXPERIMENTAL METHODS ............................................38

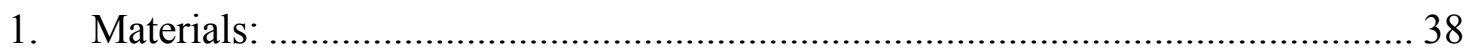

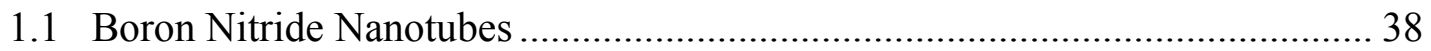

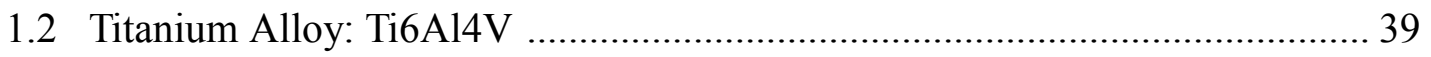

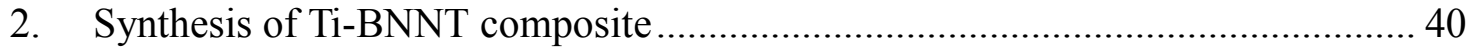

2.1 Dispersion techniques ................................................................................ 41

2.1.1 Dispersion of BNNT by wet chemistry route ......................................... 41

2.1.2 High energy induced dispersion by tip sonication ................................... 42

2.2 Pressure-assisted powder metallurgy ................................................................ 44

2.2.1 Low-temperature sintering ................................................................. 44

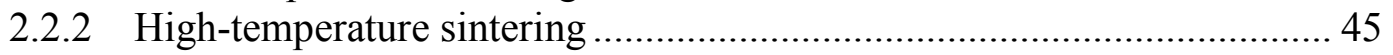

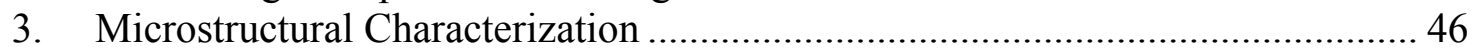

3.1 X-ray Diffraction: Identification of phases in consolidated Ti-BNNT

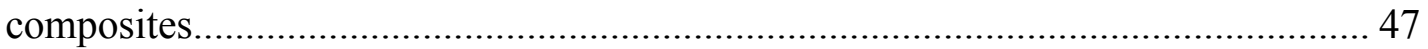

3.2 Metallography: Microstructural evolution as a function BNNT addition ........ 48

3.3 Electron microscopy: Morphology of interfacial interactions of BNNT/metal 49

4. Mechanical Characterization: Strengthening induced by BNNTs ........................ 50

CHAPTER IV: RESULTS AND DISCUSSION ………………………………………....53

1. Addressing Challenges: Dispersion of high surface energy BNNTs ......................53

1.1 Chemical dispersion of BNNTs and synthesis of composite powder................ 53 
1.1.3 Role of surfactant in the dispersion of BNNTs ........................................ 55

1.1.4 Homogeneity and dispersion of BNNTs in composite powder ................ 58

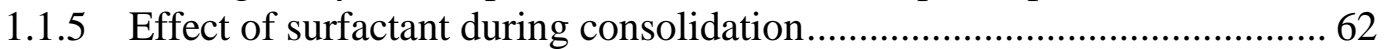

1.2 High energy induced dispersion of BNNTs by tip sonication .......................... 65

1.2.6 Role of ultrasonic waves and cavitation in breaking agglomerates and

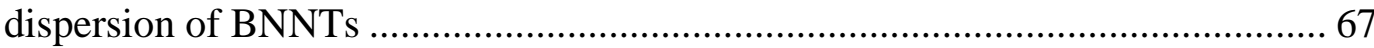

2. Addressing Challenges: Controlling thermodynamically feasible reactions ......... 70

2.1 Low-Temperature sintering of Ti64Al4V …………...................................... 70

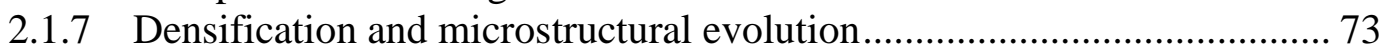

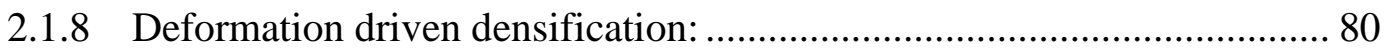

2.2 High-Temperature sintering of Ti6Al4V …………..................................... 85

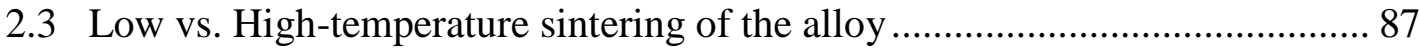

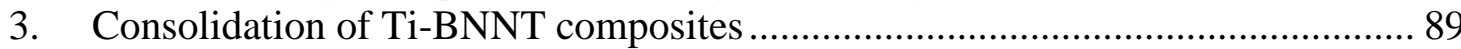

3.1 Low vs. High-temperature sintering of Ti-BNNT composites .......................... 89

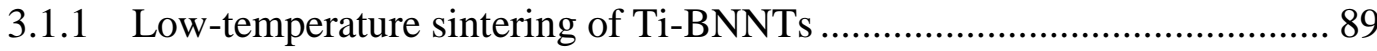

3.1.2 High-temperature sintering of Ti-BNNTs …………….......................... 97

3.2 Evaluation of mechanical properties of Ti-BNNT composites under

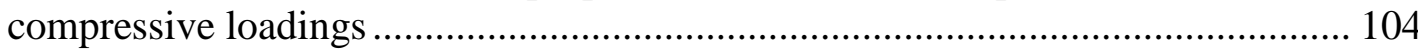

3.2.3 Mechanical properties of Ti-BNNT composites under compression ..... 104

3.2.4 Nano- and Microscale strengthening of composites .................................. 111

CHAPTER V: CONCLUSIONS .............................................................................. 118

CHAPTER VI: RECOMMENDATIONS \& FUTURE WORK …………………….... 120

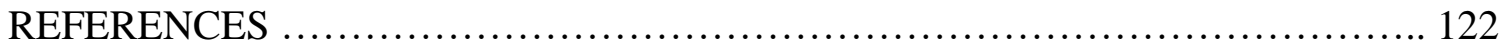




\section{LIST OF TABLES}

TABLE

PAGE

Table 1 Literature review summary of Boron Nitride Nanotube-reinforced polymer composites. 15

Table 2 Literature summary of reported ceramic matrix composites reinforced by BNNTs.

Table 3 Elemental composition of as-received Ti6Al4V powder obtained from EDS analysis

Table 4 Sintering parameters used for low-temperature sintering of Ti6Al4V

Table 5 Summary of microstructural characterization of low temperature sintered Ti6Al4V

Table 6 Summary of computed average crystallite size and microstrain using

Williamson-Hall method.

Table 7 Comparison between low and high-temperature sintering of Ti6Al4V alloys and their contribution to the manufacturing of composites 88

Table 8 Summary of compressive properties of Ti-BNNT composites sintered at high and low temperatures

Table 9 Summary of mechanical properties obtained by nanoindentation technique .... 113 


\section{LIST OF FIGURES}

FIGURE

PAGE

Figure 1 Statistical representation of a number of research papers focused on BNNTs and their areas of significant interest ${ }^{14}$.

Figure 2 Atomic representation of 2D Hexagonal Boron Nitride (h-BN) as the building block for Boron Nitride Nanotubes (BNNTs), and TEM showing the morphology of multiwalled BNNTs ${ }^{16}$.

Figure 3 (a) Ball milling experimental process to synthesize high yield BNNTs ${ }^{7}$. (b) A TEM micrograph of resulting BNNTs synthesized by ball milling process yielding nanotubes with bamboo-like morphologies ${ }^{25}$.

Figure 4 (a) BNNT fibril synthesized via a pressurized vapor/condenser method yielding $200 \mathrm{mg}$. (b) A TEM micrograph of BNNTs with 1, 3 and 5 well defined parallel walls signaling their highly crystalline nature ${ }^{27}$.

Figure 5 TEM micrograph of an $\mathrm{Al}_{2} \mathrm{O}_{3}$ reinforced matrix showing BNNTs segregation at the grain boundaries hindering the growth of ceramic grains $(0.5 \mathrm{wt} . \% \mathrm{BNNT})^{40}$. Strengthening mechanisms such as crack bridging by BNNTs in a $4 \mathrm{wt} . \%$ BNNT reinforced HA matrix, inset shows the Vickers indent with crack propagation signs ${ }^{43} \ldots 18$

Figure 6 Graphical representation of ion implantation regimes in the deposition of $\mathrm{Al}$ to produce Al-BNNT composites. (a) Demonstrates the crystalline nature (parallel lines represent walls) of the as-received BNNTs before implantation. (b) High energy regime (3) imparting the deposition of $\mathrm{Al}$ on the surface and inner tubular structure of BNNTs. (c) Medium energy regimes (2) shown by the formation of uniform Al coating on the BNNT. (d) Low energy regimes (1), in which defects are introduced to the structure ${ }^{48,49}$

Figure 7 (a)TEM micrograph of BNNT after magnetron sputtering with Al, resulting in polycrystalline coatings of $\sim 200 \mathrm{~nm}$. (b) Evidence of retained structural integrity in BNNT after Al sputtering ${ }^{50}$.

Figure 8 (a) Reacted layer on the surface of a nanotube with bamboo-like morphology after exposure to $650{ }^{\circ} \mathrm{C}$ for 1 hour. (b) Nucleation of reaction phases in the presence of a defective, broken BNNT wall ${ }^{25}$......

Figure 9 (a) TEM micrograph of a single nanotube showing localized reacted species (AlN and $\mathrm{AlB}_{2}$ ) on the surface after undergoing plasma spraying ${ }^{53}$. (b)Brittle fracture of reaction phases at the interface of an Al-BNNT mat composite manufactured by spark plasma sintering ${ }^{4}$.

Figure 10 (a) Schematic representation of the interactions between molten $\mathrm{Al}$ and the unreacted BNNT vs. the improved wetting in the presence of an AlN layer. (b) Strengthening mechanism in Al-BNNT in the presence of interfacial reactions, showing BNNTs acting as bridges. (c) ${ }^{4,52-54}$ 
Figure 11 (a) Stress-strain curves of Al-BNNT composite manufactured by the hot rolling process with strengthening mechanisms dominated by (b) BNNTs ability to restrain grain growth and promote the pile-up of dislocations along its length ${ }^{5}$..... 28

Figure 12 Comparative chart demonstrating the specific strength of multiple structural metals as a function of increasing temperatures ${ }^{12,58-60}$.

Figure 13 (a) Phase diagram of pure Titanium as a function of increasing pressure and temperature, displaying the presence of three phases: low temperature ( $\alpha$-phase), high temperature ( $\beta$-phase) and metastable $\omega$-phase requiring high pressures. (b) Crystal structures of $\alpha$ - phase Ti showing HCP crystal structure and $\beta$-phase Ti with BCC crystals $^{12,58-60}$

Figure 14 (a) Phase diagram of an $\alpha-\beta$ alloy with 6 wt.\% Al and V as alloying elements, the segmented line represents the phase transformation path of Ti6Al4V

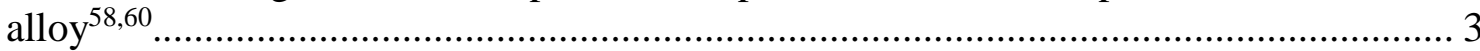

Figure 15 Gibb's free energy of formation of various $\mathrm{Ti}$ and $\mathrm{BN}$ reactions ${ }^{11,65}$............. 33

Figure 16 (a)TEM micrograph showing $\mathrm{TiB}_{2}$ reaction phases at the surface of a single nanotube coated $\mathrm{Ti}$ and exposed to $800^{\circ} \mathrm{C}$ for $30 \mathrm{~min}$. (b)Formation of $\mathrm{TiB}_{2}$ nanoparticles and $\mathrm{TiB}$ needle/whiskers after exposure of Ti-coated nanotubes to $900^{\circ} \mathrm{C}$ for 1 hour $^{47}$. 35

Figure 17 Fracture surface of Ti-BNNT composite (a) sintered at $800^{\circ} \mathrm{C}$ for $1 \mathrm{~h}$ after experiencing compressive fracture showing $\mathrm{TiB}_{2}$ as primary reaction phases and survived BNNTs, (b) composite sintered at $1000^{\circ} \mathrm{C}$ for $3 \mathrm{~h}$ showing abundant TiB whiskers as a result of Ti-BNNT reactions ${ }^{34}$.

Figure 18 As received (a) BNNT fibril balls synthesized by PVC method ${ }^{74}$, (b) SEM micrograph of entangled long and ultrafine BNNTs. Inset shows the smooth morphology of a single nanotube.

Figure 19 As-received Ti6Al4V with spherical morphology and average particle size of $88.25 \pm 4 \mu \mathrm{m}$.

Figure 20 Experimental set-up used to evaluate the bulk compressive properties.... 52

Figure 21 Pictorial representation of the as-received BNNT fibrils in the form of balls, the subsequent introduction to the aqueous medium resulted in their segregation and non-wetting as a result of the highly hydrophobic nature of BNNTs

Figure 22 Schematic representation showing the mechanism by which a non-ionic surfactant introduced in the BNNT/DI water solution results in the reduction of surface tension between the nanotube and the aqueous media.

Figure 23 (a) Resultant Ti-BNNT composite powder after drying and thermal process was completed showing residual surfactant. (b) EDS mapping of the composite powder revealed the presence of Carbon as a result of residual surfactant

Figure 24 (a) Titanium powder particle with individual BNNTs adhered at the surface of the powdered particle. (b) Evidence of BNNTs interfacing with the powder particles and surfactant 
Figure 25 EDS mapping of the as-sintered fracture surface of the Ti-BNNT composite synthesized via surfactant assisted route. Carbon as an impurity is encountered

Figure 26 TGA curve of the Pluronic F127 used in the dispersion of BNNTs, curve demonstrates major thermal degradation to occur between $200-400^{\circ} \mathrm{C}$ with residual Carbon on $1.5 \%$ of its initial weight.

Figure 27 Optical micrographs of polished and etched surfaces of the as-sintered TiBNNT composites synthesized via surfactant-assisted route showing the significant presence of voids as a result of volatile organics in residual surfactant.

Figure 28 Pictorial representation of the high energy induced dispersion of high surface energy BNNTs in acetone. The resultant composite powder shows the integrity of the nanotubes to high energy sonication and the achieved dispersion.

Figure 29 Comparative chart of pressureless and pressure assisted sintering routes used in the past for the densification of Ti6Al4V. A processing gap is identified and investigated with potential advantages for Ti-BNNT composites

Figure 30 Densification curves of low temperature sintered Ti6Al4V showing three distinct regimes of densification

Figure 31 Fracture surface of the Ti6Al4V sample sintered at $600^{\circ} \mathrm{C}$ and $300 \mathrm{MPa}$, showing poor interparticle interactions with signs of surface diffusion (necking). 76

Figure 32 SEM micrographs of as-sintered Ti6Al4V at (a) $600^{\circ} \mathrm{C}$ and pressures of 555 $\mathrm{MPa}$, as well as (b) $650^{\circ} \mathrm{C}$ and $555 \mathrm{MPa}$. Improved interparticle interactions showed near full densification of the Ti alloy.....

Figure 33 Optical micrographs of the polished and etched surfaces of Ti6Al4V sintered at low-temperature conditions.

Figure $34 \mathrm{X}$-ray Diffraction pattern of as-sintered Ti6Al4V sample as a function of sintering conditions. Shifting of peaks as a result of high sintering pressures are observed.

Figure 35 Transmission electron micrograph of Ti6Al4V specimen sintered at $750^{\circ} \mathrm{C}$ and $555 \mathrm{MPa}$ showing slip bands as hosts for dislocations.

Figure 36 (a) Densification curves comparing sintering mechanisms of high-temperature sintering and low-temperature sintering of Ti6Al4V. (b) Optical micrograph of the etched and polished surface of the alloy sintered at $950^{\circ} \mathrm{C}$ with characteristic elongated $\alpha$-grains. (c) and (d) fracture surface of the alloy sintered at high temperatures showing ductile dimples.

Figure 37 Fracture surface of as-sintered Ti-BNNT composite at $650^{\circ} \mathrm{C}$ and $555 \mathrm{MPa}$ pressures (a) showing poor sintering and particle-particle interactions, (b)restricted plastic flow by BNNTs located at the interface of the Titanium grain boundaries

Figure 38 (a) Improved fracture surface of Ti-BNNT composites sintered at $750^{\circ} \mathrm{C}$ and $555 \mathrm{MPa}$ with (b) enhanced interfacial interactions and (c) no reaction products. 
Figure 39 XRD pattern of as-sintered composite and control specimen at $750^{\circ} \mathrm{C}$ and $555 \mathrm{MPa}$ with primary peaks corresponding to $\alpha$-Ti and no reaction phases identified .. 95

Figure 40 (a) Optical micrograph of the polished and etched surface of the Ti-BNNT composite sintered at low temperatures and high pressures with globular morphologies. (b) and (c) Presence of BNNTs across grain boundaries without morphological changes.

Figure 41 (a) High resolution (HR) Transmission electron micrograph of the Ti-BNNT composites sintered at $750^{\circ} \mathrm{C}$ and $555 \mathrm{MPa}$ with evidence of twin boundaries (b) corresponding SAED pattern showing diffraction planes of twins

Figure $42 \mathrm{X}$-ray diffraction pattern of the as-sintered Ti-BNNT composite at high temperatures of $950^{\circ} \mathrm{C}$ with no presence of reacted phases in the pattern due to small quantity if any.

Figure 43 Fracture surface of the (a) Ti-BNNT composites sintered at high-temperature conditions, (b) and (c) survived BNNTs with some partial reactions of straight needle features.

Figure 44 (a) Polished and etched the surface of the high temperature sintered Ti-BNNT composite showing microstructural features. (b) and (c) Strong interactions between the BNNTs and the matrix with some reacted TiB phases near the grain boundaries.

Figure 45 (a) EDX line mapping of the surface of the Ti-BNNT composite sintered at high temperatures, with evidence of TiN as a solid solution. (b) The interface of nanotube walls with $\mathrm{d}$-spacing of $\sim 0.3-0.4 \mathrm{~nm}$ and potential reaction layer on its surface.

Figure 46 (a) Stress vs. Strain curve of Ti-BNNT and control (Ti64) specimens sintered at $750^{\circ} \mathrm{C}$. (b) the fracture surface of the composite evidencing crack-bridging by BNNTs as stress dissipation mechanism, (c) TEM micrograph showing the presence of shear band allocating dislocations and restricting their motion.

Figure 47 Fracture surface micrographs showing (a) the sheared failure surface of the composite with ripple-like features, (b) plastic shearing of Ti metal and (c) inducing plastic flow at the Ti/BNNT interfaces....

Figure 48 (a) Stress vs. Strain curve of Ti-BNNT and control (Ti64) specimens sintered at $950^{\circ} \mathrm{C}$. (b) and (c) TEM micrographs showing the presence of shear bands and dense dislocation microstructure with arrested dislocations at the interface of the BNNT and Ti

Figure 49 (a) Fracture surface of compressive failure experienced by the Ti-BNNT composite sintered at $950^{\circ} \mathrm{C}$, (b) BNNT/Ti interfaces with deformed BNNTs attesting the flexible nature of the nanotubes. (c) and (d) show the strong interfacial interactions between the nanotube and the Ti-matrix with sheared nanotubes.

Figure 50 Load-displacement curves of Ti-BNNT composites as a function of sintering conditions. 
Figure 51 (a)Load-displacement curves of Ti-BNNT composite and Ti6Al4V control specimen showing the bimodal characteristics in the presence of (b) Ti dominant

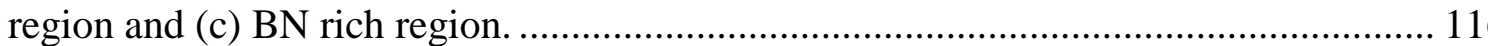




\section{CHAPTER I: Introduction}

The increasing demand for structural materials capable of combining high strength and lightweight is the driving factor for the engineering of high-performance nanoparticle reinforced metal matrix composites. The automotive, aerospace, and naval industries are some of the many industries whose contending objective is to enhance the performing efficiency of emerging technologies ${ }^{1}$. Meeting this objective entails a significant decrease in the mass of structures while maintaining mechanical functionality of the metal.

Boron Nitride Nanotubes (BNNTs) have proven great potential as reinforcing nanoparticles in low melting point metal matrices (i.e., Aluminum) by providing high strength-to-weight ratios $^{2-5}$. The main challenge revolving the reinforcement of higher melting point metal matrices such as Titanium lies in the survival of the reinforcing nanoparticles. The thermal stability of BNNTs to up to $900^{\circ} \mathrm{C}$ in the air prove their potential to reinforce Titanium matrices ${ }^{6-8}$.

The overall objective of this thesis is to synthesize high strength, and low-density Boron Nitride Nanotube (BNNT) reinforced Titanium composites by Spark Plasma Sintering. The objective will be achieved by addressing two main challenges in the introduction of BNNTs to the Titanium matrix: (i) The homogenous dispersion of high surface energy boron nitride nanotubes in the Ti matrix. And, (ii) the development of BNNT reinforced Titanium composites by powder metallurgy with controlled interfacial reactions. 


\section{Motivation}

\subsection{Demand for lightweight and high strength structural materials}

Across all engineering industries, the ever-growing quest for maximizing the efficiency of operations while minimizing the environmental footprint has become the primary goal. Reducing structural weight while maintaining mechanical performance presents a challenge to commonly found materials ${ }^{4,6,9}$. The exhaustive conditions attained in the automotive, aerospace and naval applications including high mechanical loadings, wear, elevated temperatures, and oxidizing settings have prompted the need for materials with properties exceeding those of currently used metals and alloys ${ }^{2,5,6,10}$. Titanium and its alloys possess excellent thermo-mechanical stability, corrosion resistance, and high specific strength $^{6,11}$. Yet, its mechanical performance does not meet the requirements in its entirety to present a solution to the design criteria.

The increasing interest to produce lightweight materials with superior structural properties required in automotive, aerospace and naval applications, has prompted the development of novel nanoparticle reinforced metal matrix composites ${ }^{1}$. Technological advancements in MMCs have demonstrated their potential to substitute conventional metals and alloys by achieving materials with enhanced specific strengths, wear resistance, and the advantage of tailorable properties ${ }^{1,12}$. A limiting factor in the reinforcement of Titanium matrices lies in the high reactivity of the reinforcing particles with the metal during processing involving elevated temperatures ${ }^{1,6}$. Hence, it is important that the reinforcing nanoparticle possesses thermal and chemical stability to withstand the elevated processing temperatures used in Ti matrices. 


\subsection{Boron Nitride Nanotubes (BNNTs): Potential reinforcement of Ti-matrices}

Boron Nitride Nanotubes (BNNT), a structure analogous to the well-researched carbon nanotubes (CNT), are comprised of boron and nitrogen atoms arranged in a hexagonal and layered fashion (h-BN) ${ }^{7,8}$. Its atomic configuration renders nanostructures combining a wide range of properties suitable for structural, electronic and energy applications ${ }^{3,7,8,13}$ :

- Excellent thermal stability up to $900^{\circ} \mathrm{C}$ in the air without experiencing structural or chemical modifications

- Ultra-low density (true density of $\sim 1.4 \mathrm{~g} / \mathrm{cm}^{3}$ )

- Superior mechanical stiffness with elastic modulus $>1 \mathrm{TPa}$, and strength of $\sim 60$ $\mathrm{GPa}$

- High thermal conductivity $(200-350 \mathrm{~W} / \mathrm{mK})$ and piezoelectricity

Owing to their tubular nature, the introduction of BNNTs as a reinforcing material in metal matrix composites results in strengthening of the structures by load transfer mechanisms ${ }^{4}$. Due to polarized B-N bonds, a permanent dipole allows properties such as piezoelectricity to make this material fascinating for radiation shielding and energy harvesting applications ${ }^{8}$. The thermal and oxidation stability of BNNTs (up to $900^{\circ} \mathrm{C}$ in the air) make these nanostructures favored over its carbon analogous (CNT) to reinforce metalmatrices as their processing is often carried out at elevated temperatures $\left(>500^{\circ} \mathrm{C}\right)^{7,13}$. 


\section{Research Objectives}

The overall objective of this work is to synthesize high strength, and low-density Boron Nitride Nanotube (BNNT) reinforced Titanium composites by Spark Plasma Sintering. The following are specific approaches to achieve the objective of this thesis:

- Homogenous dispersion of high surface energy BNNTs in the Ti-matrix to impart effective reinforcement of the composite - Surfactant-assisted, and high energy induced dispersion routes are used to establish a suitable method for the SPS of TiBNNT composites.

- Ti-BNNT composites synthesized with high densification and controlled interfacial reaction products are a requirement to improved mechanical properties -

a. Low-temperature sintering of Ti-BNNT composites is introduced as a promising technique to minimize thermodynamically feasible reactions at Ti/nanotube interface.

b. Spark Plasma Sintering as a fast ( 10 min) consolidation technique is used to limit the kinetics of reactions at the Ti/BNNT interface. Also, grain growth is arrested, resulting in enhanced mechanical characteristics.

- Evaluation of the bulk mechanical properties of as-sintered Ti-BNNT composites is evaluated under compressive loads - Fundamental understanding of the hierarchical stress-transfer and strengthening mechanisms between nanotubes and Ti-matrix.

The subsequent chapter, Chapter 2, provides an overview of the physical characteristics of BNNTs and recent efforts in the integration of BNNTs to metallic matrices. The advantages and lessons learned from their addition to low melting point 
metallic matrices such as Aluminum, and limited efforts in Titanium matrices are discussed. Chapter 3 outlines the detailed materials and experimental procedures utilized in the synthesis, processing, and evaluation of the Ti-BNNT composites. Chapter 4 discussed extensively and in great detail, the experimental results and implications to the strengthening mechanisms in the Ti-BNNT composites. Finally, Chapter 5 outlines the significant conclusions arrived at through this work and present recommendations for the future investigations of Ti-BNNT composites. 


\section{CHAPTER II: Literature Survey}

\section{Boron Nitride Nanotubes (BNNTs): A mechanically, chemically, and thermally}

stable nanoparticle

The physical, mechanical, and thermal properties of the one-dimensional Boron Nitride Nanotubes (BNNTs) are the main motivation for their implementation as reinforcement particles in this study. Since its predicted existence around 1994, and shortly followed by its first experimental synthesis in 1995 via arc discharge method ${ }^{14}$, BNNTs have attracted significant attention due to the exciting combination of functional properties.

Figure 1 demonstrates the rising interest of BNNT research inspiring further scientific advances for this nanomaterial.

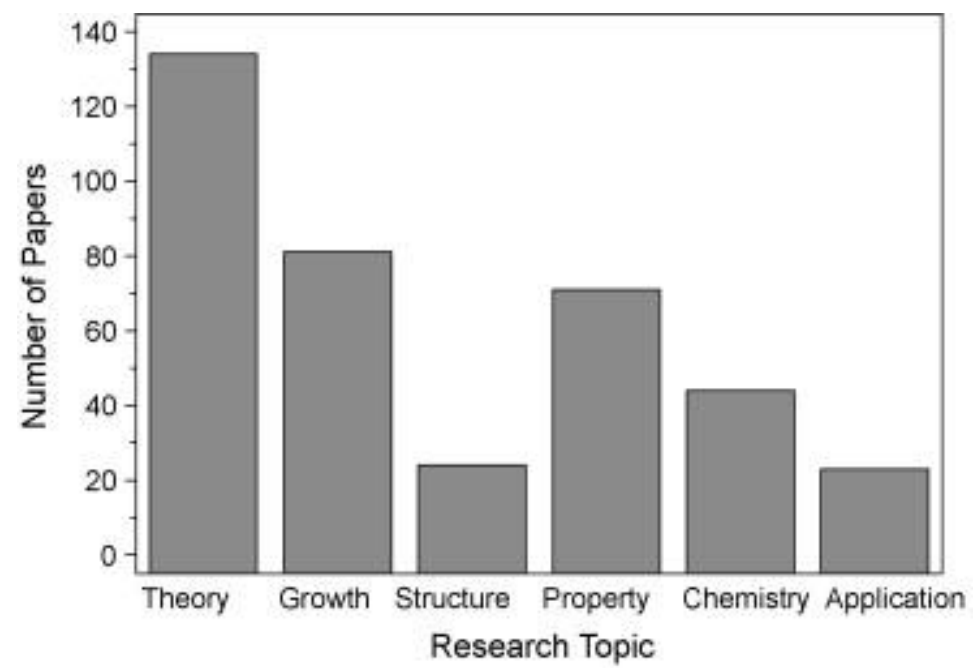

Figure 1 Statistical representation of a number of research papers focused on BNNTs and their areas of significant interest ${ }^{14}$.

Up to date, the fundamental physical, chemical, and mechanical properties of the nanotubes have been extensively researched through theory providing the foundation for BNNT related research. Experimental research of BNNTs has been limited primarily due to the extreme conditions required for the manufacturing of the nanotubes yielding small 
quantities. Hence, giving rise to research focused on the growth and manufacturing of nanotubes meeting structural, and purity requirements. Significant efforts in recent years have allowed the emergence of synthesis route yielding large quantities of high-quality BNNTs.

\subsection{Physical, chemical and mechanical properties}

The atomic structure of BNNTs consists of alternating Boron and Nitride atoms arranged in a hexagonal fashion (h-BN). The networks of h-BN atoms can be thought as rolled into cylindrical tubes to give the characteristic arrangement of nanotubular structures (Figure 2). These tubular nanostructures are often found possessing multilayers as a result of the interactions among neighboring BN layers. The walls in multiwalled BNNTs are known to have interlayer spacings of $\sim 0.34 \mathrm{~nm}$, characterizing the $\mathrm{d}$-spacing of $\mathrm{h}$ - $\mathrm{BN}$ along the 002 plane $\left(\mathrm{d}_{002}\right)$. Depending on their synthesis process, BNNTs may have diameters ranging from $2-40 \mathrm{~nm}$ and lengths of up to $1 \mu \mathrm{m}$. The covalently bonded $\mathrm{sp}^{2}$ and partially ionic $\mathrm{BN}$ bonds in BNNTs are responsible for imparting the excellent mechanical strength, thermal stability, piezoelectricity, and even high neutron absorption ${ }^{15}$.

Hexagonal Boron Nitride ( $h-B N)$

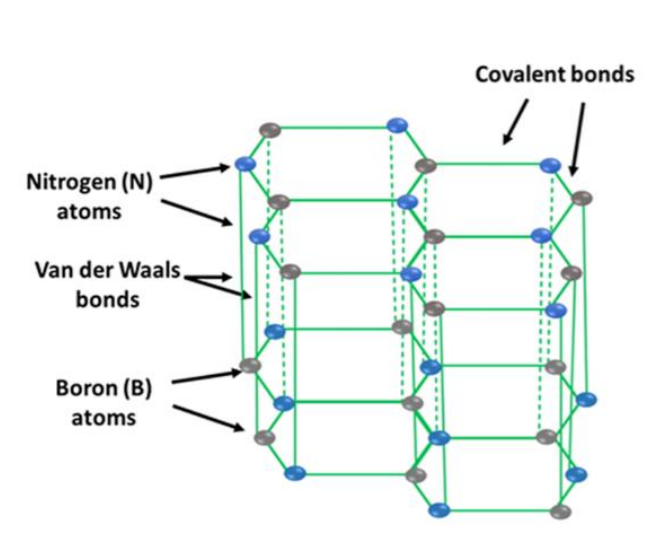

Boron Nitride Nanotube (BNNT) Multiwalled BNNTS

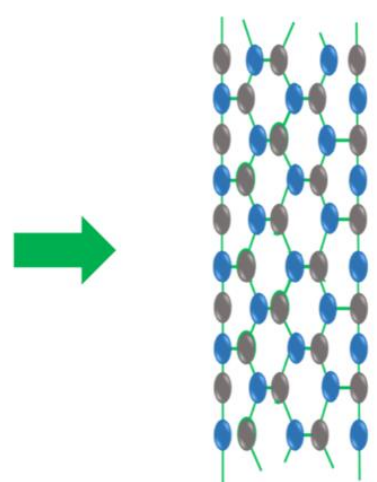

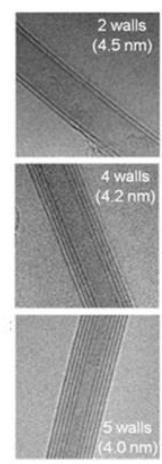

Figure 2 Atomic representation of 2D Hexagonal Boron Nitride (h-BN) as the building block for Boron Nitride Nanotubes (BNNTs), and TEM showing the morphology of multiwalled BNNTs ${ }^{16}$. 
The partially ionic B-N bonding in BNNTs is a result of the high electronegativity of N (3.04) atoms as compared to B (2.04). This provides BNNTs with electrical insulating properties and band gaps between $5-6 \mathrm{eV}^{7,17-19}$, making this nanomaterial an excellent candidate for optoelectronics ${ }^{19}$. The ability of BNNTs to transform from their electrically insulating nature to that of a semiconductor under exerted mechanical deformation has been experimentally assessed by Bai et $\mathrm{al}^{20}$. This phenomenon is known as piezoelectric behavior. In which, mechanical stimuli are bound to result in electrical discharge or vice versa. Bai et al. ${ }^{20}$ demonstrated for the first time experimentally the decreased in electrical resistance of BNNTs to up to $97 \%$ with increasing bending curvatures (curvature 1=12 $\mathrm{G} \Omega$ vs. curvature $5=0.26 \mathrm{G} \Omega)^{20}$.

Also, the structural similarity of BNNTs to the well-researched Carbon Nanotubes (CNTs), renders them with comparable thermal conductivity $(200-350 \mathrm{~W} / \mathrm{mK})$, the elastic

modulus of $\sim 1 \mathrm{TPa}$, and mechanical strengths of $\sim 60 \mathrm{GPa}^{17,19,21}$. The rising interest of BNNTs as a reinforcing nanostructure lies in their unique chemical stability and thermal stability to up to $900{ }^{\circ} \mathrm{C}$. Hence, making them excellent candidates to reinforce materials which undergo high-temperature processes and/or applications requiring the thermomechanical stability of BNNTs.

\subsection{Synthesis-dependent morphology of BNNTs: Bamboo vs. smooth}

The synthesis of BNNTs has been a subject of interest since the experimental discovery of these nanostructures. The morphology, diameter, and length of BNNTs are contingent on the synthesis process from which the BNNTs are obtained. Three 
characteristic morphologies have been identified: (i) nanotubes with capped ends, (ii) nodular (bamboo-like), and (iii) smooth cylindrical nanotubes ${ }^{7}$.

The first reported synthesis of BNNTs was performed by arc discharge method in $1995^{22}$. The process required the use of a BN precursor enclosed in a conductive tungsten anode. The arc plasma generated between the copper cathode and anode lead to the vaporization of the $\mathrm{BN}$-containing anode. In which, interactions between $\mathrm{B}$-metal/ $\mathrm{N}$-metal forming nitrides and borides are considered as catalysts for the growth of the nanotubes ${ }^{14,23}$. The BNNTs synthesized by Chopra et al. ${ }^{22}$ using arc discharge method had multiple walls, diameters between $1-3 \mathrm{~nm}$, and lengths of up to $200 \mathrm{~nm}^{22}$. Owing to the high temperatures used during arc discharge processes $(\sim 3000 \mathrm{~K})$, synthesized BNNTs are considered to have high crystallinity. Although this method proved the ability to experimentally produced carbon-free nanotubes, the produced nanotubes were characterized by having metallic capped ends as a result of the metal catalyst used and are confined to small volumes. Adaptations to this method include the use of conductive boron-containing compounds ( $\mathrm{HfB}_{2}, \mathrm{YB}_{2}, \mathrm{YB}_{6}$, etc.) as catalysts as opposed to the insulating $\mathrm{BN}$, and $\mathrm{N}_{2}$ as a protective gas and nitrogen source in the process.

In the quest to find the ideal synthesis method suitable for large scale production of BNNTs, the following have been explored:

Ball milling: A promising technique for the synthesis of BNNTs due to its ability to yield high volumes of BNNTs and potential for industrialization ${ }^{7}$. Figure 3 outlines the overall process to reach the BNNTs via a ball-milling process. The synthesis is based on the introduction of defects and/or amorphous structures in the initial precursor powder. Boron 
or h-BN precursor powders are often used for this process. In the case were a boron precursor is used as opposed to h-BN, $\mathrm{NH}_{3}$ gas is introduced as a protective gas and nitrogen source. The mechanical energy transferred during the ball milling process and the induced defects are responsible for allowing the high yield of BNNTs. Ease of modulating synthesis parameters such as time and RPM make this technique attractive for industrial production. Chen et al. $^{24}$ demonstrated the use of long milling periods as critical to stimulate the nitration between boron and the $\mathrm{NH}_{3}$ gas. Thus, producing a larger number of nucleation structures aiding nanotube formation. The ball milling process is followed by annealing of the ball milled powder at temperatures between $1000-1300^{\circ} \mathrm{C}$ in nitrogen containing environments to allow the growth of nanotubes ${ }^{21}$. The process does not require a catalyst; however, the presence of Fe species is noted as a result of the stainless-steel jars often used for the ball milling process ${ }^{25}$.

(a)

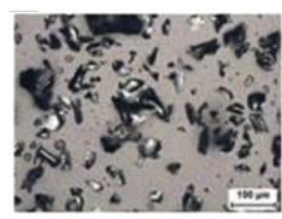

C-boron powder Average size : 60 mesh

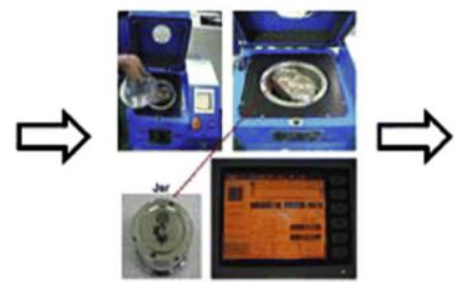

High Energy ball milling RPM : $300 \sim 1.000$

Duration: $12,24,100 \mathrm{~h}$ Gas : $\mathrm{N}_{2}$ nominal pressure
Balls \& milling vessels: STS

(b)

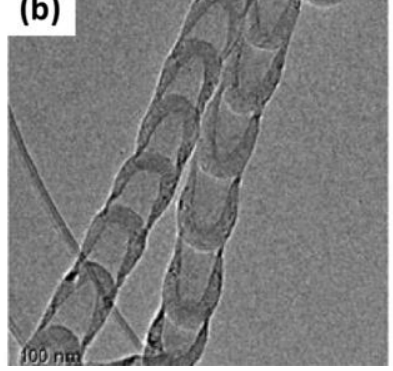

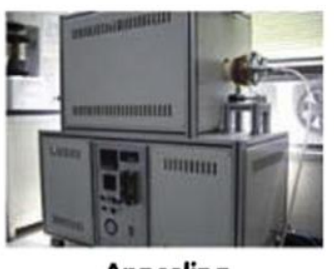

Annealing Temp: $1,100 \sim 1,200^{\circ} \mathrm{C}$ Duration: $6 \sim 24 \mathrm{~h}$ Gas type : $\mathrm{N}_{2}$

Flow rate : $2 \mathrm{~L} / \mathrm{min}$

Figure 3 (a) Ball milling experimental process to synthesize high yield BNNTs ${ }^{7}$. (b) A TEM micrograph of resulting BNNTs synthesized by ball milling process yielding nanotubes with bamboo-like morphologies ${ }^{25}$. 
BNNTs synthesized by ball milling method are often found to have diameters of $25-50$ $\mathrm{nm}$ (annealed at $1000^{\circ} \mathrm{C}$ ), lengths of up to $1 \mathrm{~mm}^{24}$. More interestingly is the resulting morphology of the BNNTs which exhibit cylindrical segments with nodules along the length, assimilating a bamboo.

Chemical Vapor Deposition (CVD): A commonly used method for the synthesis of the analogous CNTs, CVD presents advantages over other methods due to the ability to control the growth mechanism of the nanotube and have a high yield of crystalline BNNTs. The growth of BNNTs by CVD process uses a liquid or solid boron and boron nitride sources ${ }^{26}$.

The use of metal catalysts (i.e., nickel boride) was shown by Lourie et al. ${ }^{26}$ in a CVD process carried out at temperatures of $1000-1100{ }^{\circ} \mathrm{C}$ to result in multiwalled nanotubes with lengths of up to $5 \mu \mathrm{m}$ from a borazine precursor $\left(\mathrm{B}_{3} \mathrm{~N}_{3} \mathrm{H}_{6}\right)^{26}$. The synthesized nanotubes were characterized by exhibiting irregular cap morphologies, mainly attributed to the root growth mechanism of BNNTs. The root growth characterizes the morphology of BNNTs synthesized by CVD processes implementing catalysts. This growing process initiates by the diffusion of Boron atoms into the catalyst particles and the concurrent decomposition of $\mathrm{N}$ on the surface of the catalyst. This is followed by the layer-by-layer precipitation of precursors to form $\mathrm{BNNTs}^{21}$. Hence, forming nodules during the growth of BNNTs and displaying bamboo-like morphologies.

Laser ablation: A technique that allows the synthesis of BN nanotubes with perfect crystallization, consists of the heating of cubic $\mathrm{BN}$ or h-BN target by a $\mathrm{CO}_{2}$ laser to produce single-walled or multi-walled $\mathrm{BNNTs}^{24}$. The process is carried out at high nitrogen pressures between 5-15 GPa, and heating of the target by the laser reaches temperatures > 
$5000 \mathrm{~K}$ in $1 \mathrm{~min}$. The produced nanotubes are characterized by achieving diameters of up to $15 \mathrm{~nm}$ yet limited by short lengths of $<100 \mathrm{~nm}^{27}$. Also, BN nanoparticles (i.e., cones, amorphous B, etc.) as impurities in the BNNTs are often a disadvantage of this technique. Pressurized vapor/condenser method (PVC): A similar technique to laser ablation, the PVC method was introduced as a promising scalable technique for the synthesis of highquality BNNTs without introducing catalysts ${ }^{28}$. The high-temperature technique consists of the condensation of the $\mathrm{BN}$ precursor at temperatures $>4000^{\circ} \mathrm{C}$ to produce boron plume. In the presence of $\mathrm{N}_{2}$ gas pressurized at $0.7-1.4 \mathrm{MPa}$, the vaporized boron generates a narrow plume, and homogenous nucleation of B droplets are triggered upon introduction of a condenser (cooled metal wire) ${ }^{28}$. The already diffused $\mathrm{N}_{2}$ in the B plume and the presence of B droplets acting as nuclei sites allow the growth of BNNTs, forming clusters and fibrils of BNNTs ${ }^{21,28}$. The novelty of this technique lies in the extremely high yielding rate of BNNTs of $\sim 120 \mathrm{mg} / \mathrm{h}$ with high crystallinity. The BNNTs synthesized by the PVC (Figure 4) method are reported to have $2-5$ walls, lengths of at least $100 \mu \mathrm{m}$ and diameters between $2-10 \mathrm{~nm}^{28,29}$. 

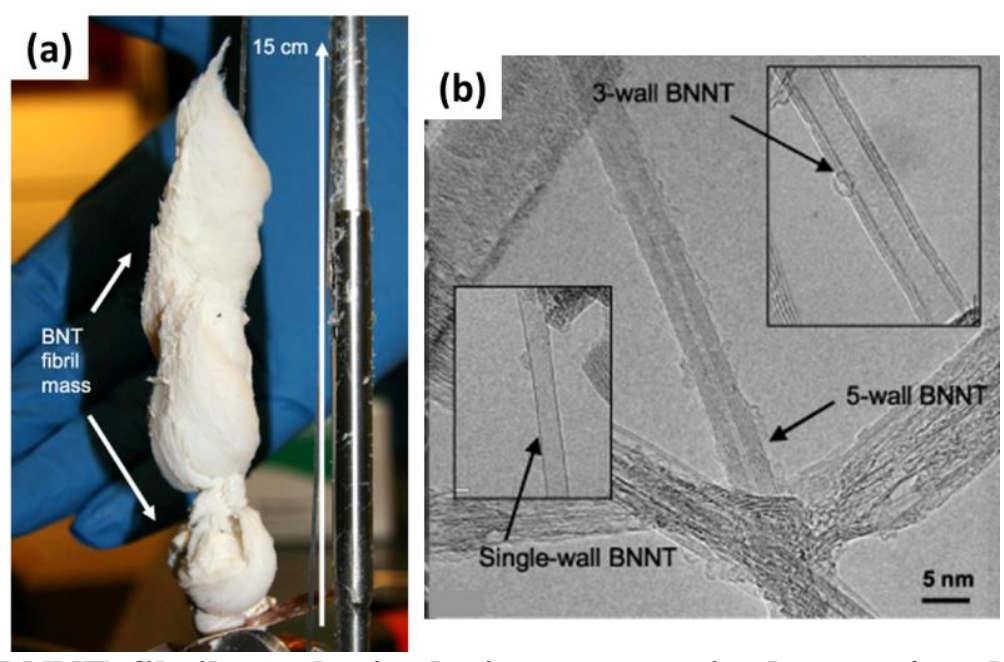

Figure 4 (a) BNNT fibril synthesized via a pressurized vapor/condenser method yielding $200 \mathrm{mg}$. (b) A TEM micrograph of BNNTs with 1, 3 and 5 well defined parallel walls signaling their highly crystalline nature ${ }^{28}$.

\subsection{Boron Nitride Nanotube-reinforced composites}

Taking advantage of the excellent mechanical, optical, electronic, thermal, and chemical properties of the BNNTs has been a continuous pursuit and challenge. The integration of BNNTs to different material matrices to produce nanocomposites with multifunctional properties has demonstrated noteworthy success. Significant efforts have demonstrated the potential and suitability of these nanotubes to enhance the mechanical and thermal properties of polymeric, and ceramic matrices.

The high thermal stability of BNNTs (up to $900^{\circ}$ ) as compared to its analogous CNTs (oxidize at $500^{\circ} \mathrm{C}$ ), allow their integration in ceramic matrices undergoing hightemperature processes $\left(\right.$ often $>1000^{\circ} \mathrm{C}$ ). It is the reported thermal and chemical stability of the BNNTs which place this nanomaterial as a potential reinforcement to highly reactive metal matrices. 


\subsubsection{BNNT reinforced polymer composites}

Table 1 displays a summary of studies where the introduction of BNNTs has resulted in the simultaneous improvement of mechanical and thermal properties of the otherwise ductile and insulating polymer matrix. The degree of reinforcement in the polymeric matrices is highly contingent on the quality of dispersion of the high surface energy nanotubes within the polymer matrix. Lahiri et al. ${ }^{30}$ demonstrated the strong bonding interactions between BNNTs with a Polylactide-polycaprolactone matrix (PLC). In which, the preferred wetting of the BNNTs by the polymer (contact angle $=71.5^{\circ}$ ) is a result of the molecular units of the polymer forming helical coils imparting strong $\pi-\pi$ interactions ${ }^{31,32}$. This phenomenon has been observed in a wide array of polymer matrices such as PLC $^{30}$, Polyaniline ${ }^{33}$, Polysterene ${ }^{29}$, etc. The extent of strengthening in PLC matrices reinforced by BNNTs is of up to $109 \%$ the strength of the matrix ${ }^{30}$ These findings outline the requirement for the effective mechanical reinforcement of BNNTs in polymer matrices as: (i) homogenous dispersion of nanotubes, and (ii) interfacial bonding (matrix/nanotube). Fulfilling the two aforementioned requirements would ideally maximize the shearing strength between the matrix and the nanotube, as described by the shear lag model of fiber reinforced polymer composites given the correct aspect ratio. In addition to mechanically stable BNNT-based polymer composites, the introduction of BNNTs results in the development of thermally-conductive networks within the insulating polymer matrices. For instance, a high content PMMA reinforced matrix (24 wt.\% BNNT) was found to yield thermal conductivities $21 \times$ larger $\left(3.16 \mathrm{Wm}^{-1} \mathrm{~K}^{-1}\right)$ than that of its polymer matrix. Hence resolving the potential of BNNT reinforced polymer composites for applications demanding strength and faster heat-releasing rates properties. 
Table 1 Literature review summary of Boron Nitride Nanotube-reinforced polymer composites

\begin{tabular}{|c|c|c|c|c|c|c|}
\hline $\begin{array}{l}\text { Polymer } \\
\text { Matrix } \\
\end{array}$ & $\begin{array}{l}\text { BNNT } \\
\text { wt.\% }\end{array}$ & $\begin{array}{c}\text { Morphology of } \\
\text { BNNTs } \\
\end{array}$ & Method & $\begin{array}{l}\text { Processing } \\
\text { conditions }\end{array}$ & Main Properties & Ref. \\
\hline Epoxy & $2-32$ & $\begin{array}{l}\text { Smooth, } \\
\text { Buckypaper }\end{array}$ & $\begin{array}{l}\text { Infiltration, } \\
\text { chemical } \\
\text { functionalization }\end{array}$ & $\begin{array}{l}\text { Oven cured at } \\
120^{\circ} \mathrm{C} \text { for } 2 \mathrm{hrs} \text {. }\end{array}$ & $\begin{array}{c}\text { Young's modulus: } 4.1-7.7 \mathrm{GPa} \\
\text { (41-100 \% reinforcement) } \\
\text { Tensile Strength: } 42 \mathrm{MPa}(60 \% \\
\text { reinforcement) } \\
\text { Strain at failure: } 0.6 \%\end{array}$ & 34-37 \\
\hline $\begin{array}{l}\text { Polyurethane } \\
\text { (PU) }\end{array}$ & 75 & $\begin{array}{l}\text { Smooth, } \\
\text { Buckypaper }\end{array}$ & $\begin{array}{l}\text { One-step } \\
\text { infiltration }\end{array}$ & $\begin{array}{l}\text { Cured at room } \\
\text { temperature }\end{array}$ & $\begin{array}{c}\text { Young's modulus: } 0.5 \mathrm{GPa} \\
\text { Tensile Strength: } 5 \mathrm{MPa} \\
\text { Strain at failure: } 1.8 \%\end{array}$ & 34 \\
\hline $\begin{array}{l}\text { Polystyrene } \\
\text { (PS) }\end{array}$ & $1-35$ & $\begin{array}{c}\text { Smooth, } \\
\text { diameter: } 40-50 \\
\text { nm, length } 10 \\
\mu \mathrm{m}\end{array}$ & $\begin{array}{l}\text { Solvent } \\
\text { dispersed }\end{array}$ & $\begin{array}{l}\text { Cured at } 60^{\circ} \mathrm{C} \\
\text { for } 4 \mathrm{hrs} ., \text { hot } \\
\text { pressing at } 80^{\circ} \mathrm{C}, \\
60 \mathrm{MPa} \text { for } 2 \mathrm{hrs} .\end{array}$ & $\begin{array}{l}\text { Young's Modulus: } 2.57-2.90 \mathrm{GPa} \\
\text { (7-20 \% reinforcement) } \\
\text { Tensile Strength: } 19-20.4 \mathrm{MPa} \\
\text { Thermal conductivity: } 3.61 \mathrm{Wm}^{-1} \mathrm{~K}^{-} \\
{ }^{1}(20 \times \text { increase })\end{array}$ & 29,38 \\
\hline $\begin{array}{l}\text { Polymethyl } \\
\text { methacrylate } \\
\text { (PMMA) }\end{array}$ & 24 & $\begin{array}{c}\text { Smooth, } \\
\text { diameter: up to } \\
50 \mathrm{~nm} \text {, length: } \\
4-10 \mu \mathrm{m}\end{array}$ & $\begin{array}{l}\text { Solvent } \\
\text { dispersed }\end{array}$ & $\begin{array}{l}\text { Hot pressing at } \\
80^{\circ} \mathrm{C}, 60 \mathrm{MPa} \text { for } \\
2 \mathrm{hrs} .\end{array}$ & $\begin{array}{l}\text { Thermal conductivity: } 3.16 \mathrm{Wm}^{-1} \mathrm{~K}^{-} \\
{ }^{1}(21 \times \text { increase })\end{array}$ & 38 \\
\hline $\begin{array}{l}\text { Polylactide-- } \\
\text { polycaprolacto } \\
\text { ne (PLC) }\end{array}$ & $2-5$ & $\begin{array}{l}\text { diameter: } 32- \\
145 \mathrm{~nm}, \text { length: } \\
0.43-5.8 \mu \mathrm{m}\end{array}$ & $\begin{array}{c}\text { Bath } \\
\text { sonication/solve } \\
\text { nt dispersed }\end{array}$ & $\begin{array}{l}\text { Cured at room } \\
\text { temperature for } \\
24 \text { hrs. }\end{array}$ & $\begin{array}{l}\text { Young's Modulus: } 0.16-1.19 \mathrm{GPa} \\
\text { (100-1370 \% increase) } \\
\text { Tensile strength: } 4.98-5.59 \mathrm{MPa} \\
\text { (87-109 \% increase) } \\
\text { Strain at break: } 240 \%\end{array}$ & 30 \\
\hline Polyimide & 2 & $\begin{array}{l}\text { Smooth, } \\
\text { diameter: } 1-10 \\
\text { nm, length: }\end{array}$ & $\begin{array}{c}\text { In-situ } \\
\text { polymerization } \\
\text { under shear and } \\
\text { sonication } \\
\end{array}$ & $\begin{array}{l}\text { Cured at } 100^{\circ} \mathrm{C}- \\
250^{\circ} \mathrm{C} \text { in } \mathrm{N}_{2}\end{array}$ & $\begin{array}{l}\text { Young's modulus: } 2.5 \mathrm{GPa}(8 \% \\
\text { increase) } \\
70 \% \text { transparence in } 650 \mathrm{~nm} \\
\text { wavelengths }\end{array}$ & 39 \\
\hline
\end{tabular}




\begin{tabular}{|c|c|c|c|c|c|c|}
\hline $\begin{array}{l}\text { Polymer } \\
\text { Matrix }\end{array}$ & $\begin{array}{l}\text { BNNT } \\
\text { wt. \% }\end{array}$ & $\begin{array}{c}\text { Morphology of } \\
\text { BNNTs }\end{array}$ & Method & $\begin{array}{l}\text { Processing } \\
\text { conditions }\end{array}$ & Main Properties & Ref. \\
\hline $\begin{array}{c}\text { Polyvinyl } \\
\text { butyral (PVB) }\end{array}$ & 18 & $\begin{array}{c}\text { Smooth, } \\
\text { diameter: } 40-50 \\
\text { nm, length } 10 \\
\mu \mathrm{m}\end{array}$ & $\begin{array}{l}\text { Solvent } \\
\text { dispersed }\end{array}$ & $\begin{array}{l}\text { Hot pressing at } \\
80^{\circ} \mathrm{C}, 60 \mathrm{MPa} \text { for } \\
2 \mathrm{hrs} .\end{array}$ & $\begin{array}{c}\text { Thermal conductivity: } 1.81 \mathrm{Wm}^{-1} \mathrm{~K}^{-} \\
1 \text { ( } 7.5 \times \text { increase }) \\
\text { Microhardness: } 36.29 \mathrm{MPa}\end{array}$ & 38 \\
\hline $\begin{array}{l}\text { Poly (ethylene- } \\
\text { vinyl acetate) } \\
\text { (PEVA) }\end{array}$ & $\begin{array}{c}37 \\
\text { wt. } \%\end{array}$ & $\begin{array}{c}\text { Smooth, } \\
\text { diameter: } 40-50 \\
\text { nm, length } 10 \\
\mu \mathrm{m}\end{array}$ & $\begin{array}{l}\text { Solvent } \\
\text { dispersed }\end{array}$ & $\begin{array}{l}\text { Hot pressing at } \\
80^{\circ} \mathrm{C}, 60 \mathrm{MPa} \text { for } \\
2 \mathrm{hrs} .\end{array}$ & $\begin{array}{c}\text { Thermal conductivity: } 2.50 \mathrm{Wm}^{-1} \mathrm{~K}^{-} \\
1 \text { (14.7× increase) } \\
\text { Microhardness: } 138.3\end{array}$ & 38 \\
\hline $\begin{array}{c}\text { Polyvinyl } \\
\text { alcohol (PVA) }\end{array}$ & $\begin{array}{r}1-10 \\
\text { wt. } \%\end{array}$ & Not reported & $\begin{array}{c}\mathrm{H}_{2} \mathrm{O}_{2} \\
\text { functionalization } \\
\text {, aqueous } \\
\text { dispersion } \\
\end{array}$ & Electrospinning & $\begin{array}{l}\text { Thermal conductivity: } 0.17-0.38 \\
\mathrm{Wm}^{-1} \mathrm{~K}^{-1}(6-137 \% \text { increase })\end{array}$ & 40 \\
\hline
\end{tabular}




\subsubsection{BNNT reinforced ceramic composites}

Few studies have explored the potentials of introducing BNNTs into ceramic matrices (Table 2). The thermal stability of BNNTs (up to $900^{\circ} \mathrm{C}$ in the air) presents a significant advantage to carbon-based nanofillers in the reinforcement of ceramic matrices. The analogous carbon-based nanoparticles (CNTs) are found to experience degradation at temperatures below $500^{\circ} \mathrm{C}$ and hence considered not suitable for the reinforcement of ceramic matrices.

The introduction of BNNTs to ceramic matrices has been limited to $\mathrm{Al}_{2} \mathrm{O}_{3}, \mathrm{Si}_{3} \mathrm{~N}_{4}$, Hydroxyapatite (HA), Barium calcium aluminosilicate glass (BCAS) and Silicon oxycarbide (SiOC) ${ }^{41-46}$. The noticeable improvement to the toughness and flexural strength of the composites with BNNTs is mainly attributed to the grain size reduction observed. Hall-Petch relationship is a well-known mathematical relationship which relates the increase in yield strength and/or hardness of a material with the decrease in grain size. The reduction in grain size in BNNT-ceramic composites was attributed to the ability of nanotubes to constrain the diffusion of mass among grains of the ceramic matrix (Figure $5)$. 

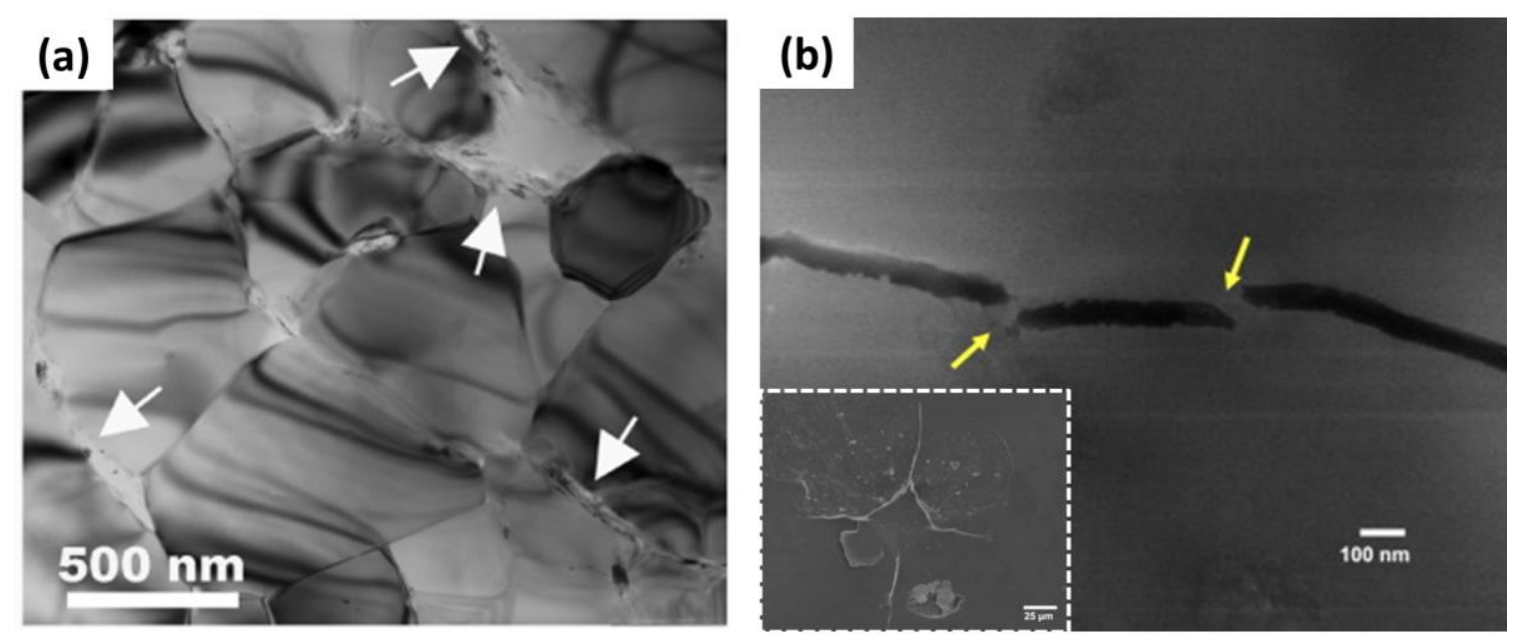

Figure 5 TEM micrograph of an $\mathrm{Al}_{2} \mathrm{O}_{3}$ reinforced matrix showing BNNTs segregation at the grain boundaries hindering the growth of ceramic grains $(0.5 \mathrm{wt} . \% \text { BNNT })^{41}$. Strengthening mechanisms such as crack bridging by BNNTs in a 4 wt.\% BNNT reinforced HA matrix, inset shows the Vickers indent with crack propagation signs ${ }^{44}$.

Given the inertness of the species involved, bonding between the ceramic matrix and the nanotubes is purely by Van der Waals bonds. Hence, strengthening is dominated by the interfacial lattice arrangement (nanotube/ceramic), controlling the work required to separate the nanotube from the matrix. Lahiri et al. ${ }^{44}$ showed interfacial strengths between $0.35-3 \mathrm{GPa}$ in a BNNT reinforced HA ceramic matrix. Where the energy required for a BNNT to be pulled-out from the HA matrix is $2-100 \times$ greater than that for fracture of HA to occur ${ }^{44}$. The ability of the nanotubes to bear a high level of stresses were observed by crack bridging and deflection of cracks mechanisms, widely known as high energy dissipating mechanisms for reinforced matrices (Figure 5). 
Table 2 Literature summary of reported ceramic matrix composites reinforced by BNNTs.

\begin{tabular}{|c|c|c|c|c|c|c|}
\hline $\begin{array}{l}\text { Ceramic } \\
\text { Matrix }\end{array}$ & $\begin{array}{l}\text { BNNT } \\
\text { wt. \% }\end{array}$ & $\begin{array}{ll}\text { Morphology of } \\
\text { BNNTs }\end{array}$ & Method & $\begin{array}{l}\text { Processing } \\
\text { conditions } \\
\end{array}$ & Main Properties & Ref. \\
\hline $\mathrm{Al}_{2} \mathrm{O}_{3}$ & $0.5-2.5$ & $\begin{array}{l}\text { Diameter: } 60 \mathrm{~nm} \text {, } \\
\text { length: }>10 \mu \mathrm{m}\end{array}$ & $\begin{array}{l}\text { polyvinylpyrr } \\
\text { olidone (PVP) } \\
\text { aided } \\
\text { dispersion }\end{array}$ & $\begin{array}{l}\text { SPS at } 1500^{\circ} \mathrm{C} \\
\text { for } 3 \text { min, hot } \\
\text { pressed }\end{array}$ & $\begin{array}{l}\text { Young's modulus: } 359-379 \mathrm{GPa} \\
\text { Microhardness: } 14.5-19.1 \quad(10.4 \% \\
\text { reinforcement) } \\
80 \% \text { grain size reduction } \\
\text { Flexural strength: } 436.1-532.1 \mathrm{MPa}\end{array}$ & $41-43$ \\
\hline $\mathrm{Si}_{3} \mathrm{~N}_{4}$ & 0.5 & $\begin{array}{l}\text { Diameter: } 60 \mathrm{~nm} \text {, } \\
\text { length: }>10 \mu \mathrm{m}\end{array}$ & $\begin{array}{l}\text { polyvinylpyrr } \\
\text { olidone (PVP) } \\
\text { aided } \\
\text { dispersion }\end{array}$ & $\begin{array}{l}\text { SPS at } 1600^{\circ} \mathrm{C} \\
\text { for } 5 \mathrm{~min}\end{array}$ & $\begin{array}{l}\text { Young's modulus: } 273 \mathrm{GPa} \\
\text { Microhardness: } 15.5 \mathrm{GPa}\end{array}$ & 41 \\
\hline $\begin{array}{l}\text { Hydroxyapatite } \\
\text { (HA) }\end{array}$ & 4 & $\begin{array}{l}\text { Bamboo type, } \\
\text { diameter: } 10-145 \\
\text { nm, length: } 0.43- \\
5.8 \mu \mathrm{m}\end{array}$ & $\begin{array}{l}\text { Ultrasonicatio } \\
\mathrm{n} \text { dispersed }\end{array}$ & $\begin{array}{l}\text { SPS at } 1099.85 \\
{ }^{\circ} \mathrm{C} \text { and } 70 \mathrm{MPa} \\
\text { for } 5 \mathrm{~min}\end{array}$ & $\begin{array}{l}\text { Young's modulus: } 205 \mathrm{GPa}(20 \% \\
\text { increase) } \\
\text { Hardness: } 12 \quad \mathrm{GPa} \quad(129 \% \\
\text { reinforcement) } \\
\text { Fracture toughness: } 1.6 \mathrm{MPa} \mathrm{m} \mathrm{m}^{0.5} \\
\text { (86\% higher) } \\
\text { Crack bridging by BNNTs }\end{array}$ & 44 \\
\hline $\begin{array}{l}\text { Barium calcium } \\
\text { aluminosilicate } \\
\text { glass (BCAS) }\end{array}$ & 4 & $\begin{array}{l}\text { Diameter: } 10-40 \\
\mathrm{~nm} \text {, length: }>10 \\
\mu \mathrm{m}\end{array}$ & $\begin{array}{l}\text { Ball milled for } \\
24 \mathrm{hrs} \text {. }\end{array}$ & $\begin{array}{l}\text { Hot pressed at } \\
630^{\circ} \mathrm{C} \text { and } 10 \\
\mathrm{MPa} \text { for } 15 \mathrm{~min}\end{array}$ & $\begin{array}{l}\text { Flexure strength: } 92 \mathrm{MPa}(90 \% \\
\text { reinforcement) } \\
\text { Fracture toughness: } 0.69 \mathrm{MPa}(35 \% \\
\text { increase) }\end{array}$ & 45 \\
\hline $\begin{array}{l}\text { Silicon } \\
\text { oxycarbide } \\
\text { (SiOC) }\end{array}$ & $0.25-2$ & $\begin{array}{l}\text { Diameter: } 10-40 \\
\text { nm, length: }>10 \\
\mu \mathrm{m}\end{array}$ & $\begin{array}{l}\text { Dissolve } \\
\text { BNNTs in } \\
\text { cross-linked } \\
\text { solution }\end{array}$ & $\begin{array}{l}\text { Pyrolysis of } \\
\text { tetravinyl } \\
\text { cyclotetrasiloxa } \\
\text { ne at } 1000^{\circ} \mathrm{C} \text { for } \\
10 \text { hrs. }\end{array}$ & $\begin{array}{l}\text { Efficiency of electrode: } 46.9-50 \% \\
\text { Charge capacity: } 410 \mathrm{mAhg} \mathrm{Ag}^{-1} \\
\text { Specific capacitance: } 78.93 \mathrm{Fg}^{-1} \\
86 \% \text { cyclic retention after } 185 \\
\text { cycles }\end{array}$ & 46 \\
\hline
\end{tabular}




\subsection{BNNT reinforced metal composites}

The resistance of BNNTs to experience oxidation in the presence of temperatures $\left(\sim 900^{\circ} \mathrm{C}\right)$ exceeding those of the comparable carbon nanotubes $\left(500^{\circ} \mathrm{C}\right)$, displays their potential as reinforcing nanoparticles of metallic matrices with elevated processing temperatures involved $\left(>600^{\circ} \mathrm{C}\right)$. The extremely low density of the BNNTs $\left(\sim 1.4 \mathrm{~g} / \mathrm{cm}^{3}\right)$ in combination with the excellent mechanical properties and thermal stability have prompted their addition to metallic matrices. With the continuous desire to develop a generation of materials capable of retaining mechanical strength and low densities, a matrices of interest include Aluminum, Magnesium, and Titanium. Aluminum has been the metal under continuous scrutiny, due to its low melting point $\left(\sim 660^{\circ} \mathrm{C}\right)$, has demonstrated the feasibility

of the integration of BNNTs to the metallic matrix. Recent studies ${ }^{47,48}$, have introduced Titanium as a matrix with a high melting point in efforts to evaluate the suitability of BNNTs in highly reactive metals undergoing elevated temperature processes.

\section{Structural stability of BNNTs}

Processing routes involving several thermal schedules, high energy bombarding, and high pressures have been some of the exhaustive thermo-mechanical processes withstood by BNNTs in the manufacturing of Aluminum-BNNT (Al-BNNT) composites.

Novel routes for the manufacturing of Al-BNNT composites included the deposition of metallic particles by high energy bombardment as it is in ion implantation, and magnetron sputtering. Obraztsova et al. ${ }^{49,50}$ evaluated the structural changes experienced by CVD-synthesized BNNTs after implantation of Al particles via high energy (acceleration voltage: $10-30 \mathrm{kV}$ ) ion implantation methods. The processing route is 
presented as a promising synthesis technique in which the degree of defects and coating quality are a function of acceleration voltage used. Three implantation regimes were identified during processing (Figure 6):

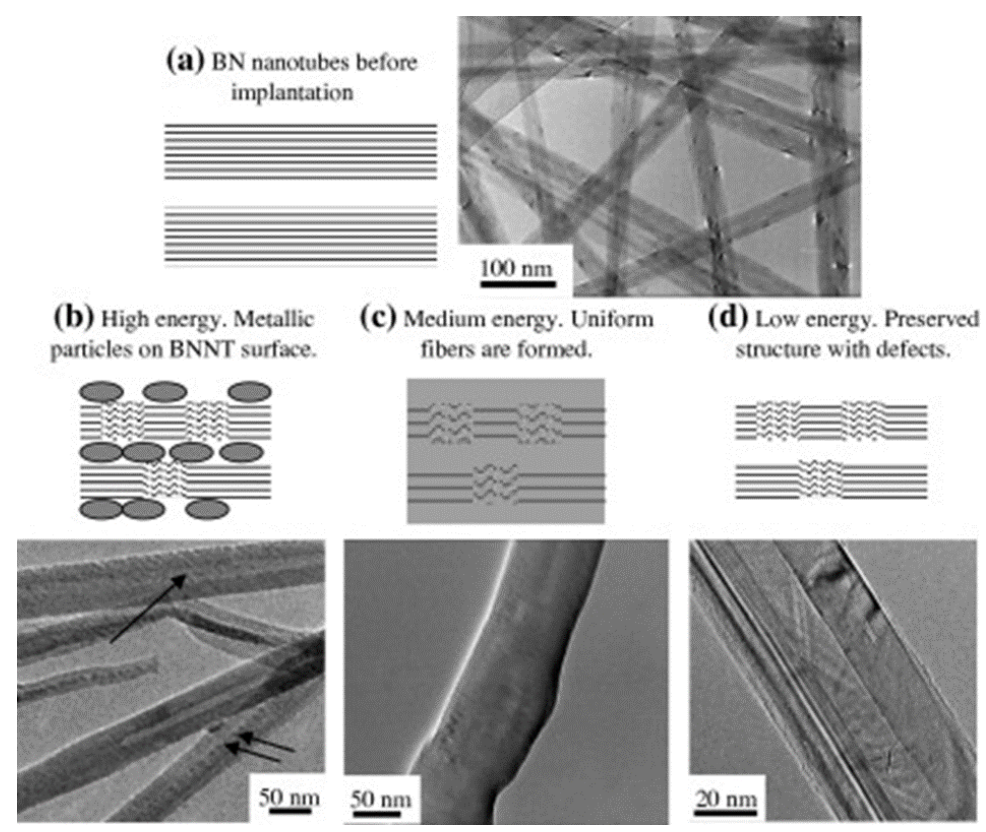

Figure 6 Graphical representation of ion implantation regimes in the deposition of $\mathrm{Al}$ to produce Al-BNNT composites. (a) Demonstrates the crystalline nature (parallel lines represent walls) of the as-received BNNTs before implantation. (b) High energy regime (3) imparting the deposition of $\mathrm{Al}$ on the surface and inner tubular structure of BNNTs. (c) Medium energy regimes (2) shown by the formation of uniform Al coating on the BNNT. (d) Low energy regimes (1), in which defects are introduced to the structure ${ }^{49,50}$.

(1) Low acceleration voltage $(10 \mathrm{kV})$, induces numerous defect in the walls of the nanotubes forming partially amorphous structures.

(2) Low-medium acceleration voltage $(10-20 \mathrm{kV})$, results in the uniform coating of BNNTs by Al metal.

(3) High acceleration voltage (30 kV), induces the implantation of metal particles on the surface and inside the nanotube as a result of high irradiated energy causing the rupture of BNNT walls. 
Under low-medium regime conditions $(10-20 \mathrm{kV}, 10 \mathrm{~mA})$, Obraztsova et al. ${ }^{49,50}$ demonstrated the capability of the technique to produce uniformly coated BNNTs. In addition to ion implantation, Yamaguchi et al. ${ }^{51}$ demonstrated the structural stability of BNNTs after been bombarded by high energy $\mathrm{Al}$ particles in a magnetron sputtering process. The flexible and robust structure of BNNTs, capable of withstanding high stresses, was retained after sputtering producing composites with polycrystalline Al coatings with thicknesses of up to $200 \mathrm{~nm}$ (Figure 7). The study demonstrated that even after experiencing high levels of energy, no interfacial reactions were introduced during the sputtering process ${ }^{51}$.

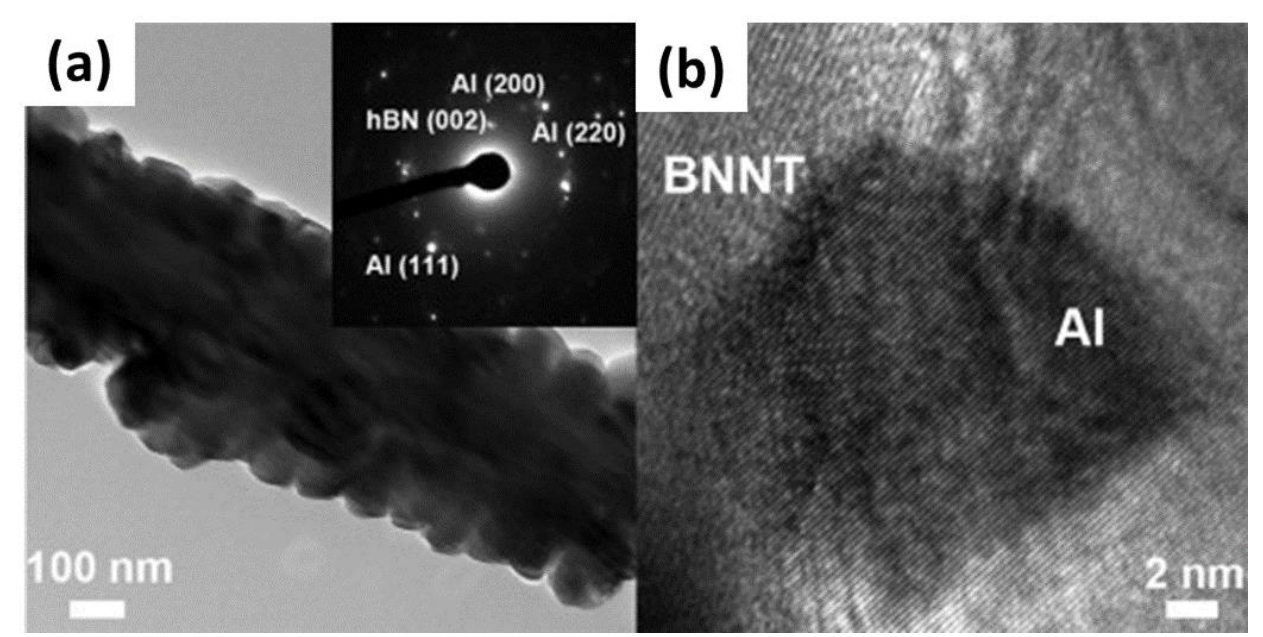

Figure 7 (a)TEM micrograph of BNNT after magnetron sputtering with Al, resulting in polycrystalline coatings of $\sim 200 \mathrm{~nm}$. (b) Evidence of retained structural integrity in BNNT after Al sputtering ${ }^{51}$.

Similarly, processing of Al-BNNT composites by spark plasma sintering (SPS), hot rolling, and high-pressure torsion have been adopted ${ }^{4,52,53}$. Powder metallurgy techniques such as spark plasma sintering present an advantage over high-energy implantation techniques due to the ease of powder preparation and short periods $(10-30 \mathrm{~min})$ required for densification. SPS is a widely used technique for the rapid consolidation of powders by 
introducing simultaneous heat and high pressures. The technique was explored by Lahiri et al..$^{53}$, Nautiyal et al. ${ }^{4}$, and Yamaguchi et al. ${ }^{52}$ in which nanotubes were able to withstand thermo-mechanical stresses (up to $600^{\circ} \mathrm{C}, 80 \mathrm{MPa}, 1 \mathrm{~h}$ ) induced by SPS and retain their structural integrity. The integrity of the nanotubes was further evidenced at even higher pressures of $\sim 2.5 \mathrm{GPa}$ at room temperature during high-pressure torsion ${ }^{52}$.

Moreover, the performance of the nanotubular structures was challenge by Nautiyal et al. $^{54}$ in synthesizing Al-BNNT composites via Plasma spray technique. In which, a combination of the high temperatures induced by the plasma promotes the melting of the metal composite powder and produces a coating upon striking a metallic substrate. The solidification of the imparted splats is reported to occur in a non-equilibrium fashion. The nanotubes are found to retain their tubular structures. Similarly, Nautiyal et al ${ }^{55}$ proved the nanotubes suitable for equilibrium solidification processes such as casting.

\section{Interfacial interactions between BNNT and metal}

Contingent with the structural stability of the nanotubes, the chemical reactivity of the nanotubes with the metal during processing is a critical factor to imparting effective mechanical reinforcement. A significant drawback in the addition of CNTs to metallic matrices is their low-temperature stability $\left(\sim 500^{\circ} \mathrm{C}\right)$, and high reactivity with metal matrices resulting in the partial or complete transformation to brittle carbide species.

The involved problem with the development of interfacial reactions between the metal and the BNNTs lies in the formation of defects and brittle species inducing lattice strains. Although interfacial reactions are considered to pose a negative outcome in the reinforcement of metal matrices, this is limited by the thickness of the reaction products. 
Thick layers of reaction products $(>100 \mathrm{~nm})$ have shown their detrimental effect on the mechanical properties and premature failure in CNT-metal composites, and more recently in Al-BNNT composites. On the other hand, thin reaction layers not exceeding $20 \mathrm{~nm}$ in thickness have been sought for as beneficial to the wetting of the nanotubes with the metal. Thus, reducing the hydrophobic nature of the nanotubes and improving the interfacial adhesion of the nanotube to the metal matrix.

The requirement of minimized chemical reactions at the nanotube/metal interface is highly dependent on the thermal processing in the manufacturing of BNNT-based metal matrix composites. The thermal decomposition of the nanotubes to form interfacial reactions with $\mathrm{Al}$ during thermal processes such as casting, conventional and spark plasma sintering and plasma spray has been shown in previous studies. The formation of AlN, $\mathrm{AlB}_{2}$, and $\mathrm{Al}(\mathrm{BNO})$ species are a result of thermodynamically feasible conditions present during processing. This thermally activated phenomenon is limited by the kinetics of reactions (long periods required), and the morphology of BNNTs (smooth vs. bamboo/segmented). Lahiri et al. ${ }^{25}$ demonstrated the formation of a thin reacted layer $(0.35-2.3 \mathrm{~nm})$ only after exposure of the Al-BNNt (5 wt. \%) to temperatures of $650^{\circ} \mathrm{C}$ for 1 hour (Figure 8). 

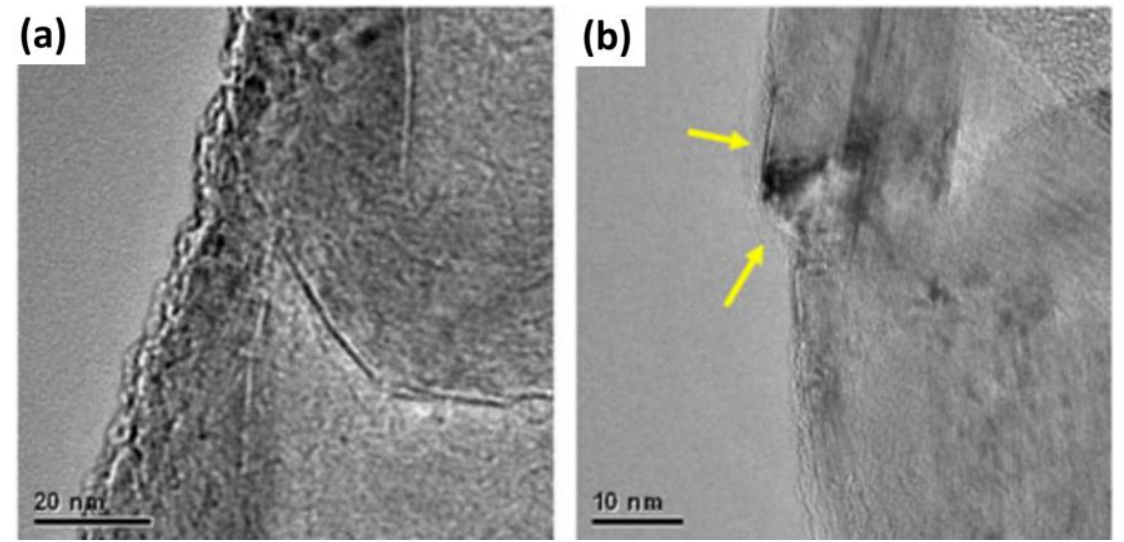

Figure 8 (a) Reacted layer on the surface of a nanotube with bamboo-like morphology after exposure to $650^{\circ} \mathrm{C}$ for 1 hour. (b) Nucleation of reaction phases in the presence of a defective, broken BNNT wall ${ }^{25}$.

The reacted layer revealed the formation of $\mathrm{AlN}$ and $\mathrm{AlB}_{2}$ crystals at the interface as a result of diffusion of $\mathrm{N}(\mathrm{AlN})$ into the $\mathrm{Al}$ matrix and $\mathrm{Al}$ into the $\mathrm{BN}\left(\mathrm{AlB}_{2}\right)$. Also, morphological features and defects in the surface of the nanotubes were found to act as nucleation sites for the reactions to occur.

This was later corroborated by Nautiyal et al. ${ }^{4,54,55}$ in the manufacturing of AlBNNT composites by plasma spray conditions, casting and spark plasma sintering. In which, solidification by plasma spray resulted in localized reactions of $\mathrm{AlB}_{2}$ and $\mathrm{AlN}$ as a result of the rapid thermal exposure $(<1 \mathrm{~ms})$ (Figure $9 \mathrm{a})^{30}$. On the contrary, the casting of the BNNT composite provided a kinetically feasible condition for reactions to occur and form a uniform AlN layer of $\sim 4-5 \mathrm{~nm}$ thick $^{55}$. The feasibility of reactions was also observed in pressure aided sintering processes, where the fracture surface demonstrated the brittle cracking of the reacted species (Figure $9 b)^{4}$. 

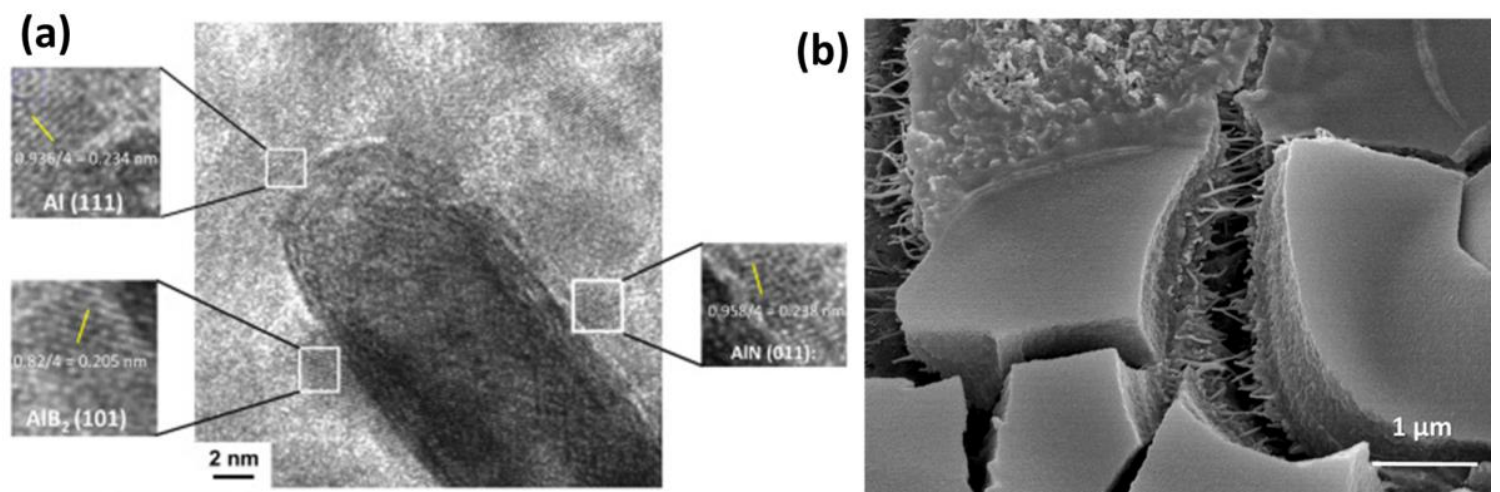

Figure 9 (a) TEM micrograph of a single nanotube showing localized reacted species (AIN and $\mathrm{AlB}_{2}$ ) on the surface after undergoing plasma spraying ${ }^{54}$. (b)Brittle fracture of reaction phases at the interface of an Al-BNNT mat composite manufactured by spark plasma sintering ${ }^{4}$.

\section{Enhanced mechanical properties}

The significance of the limited reaction products at the interface of the nanotube and the matrix is portrayed in the mechanical properties of the composites. Theoretically, the inclusion of BNNTs to metallic matrices is bound to result in effective properties higher than those of the ductile matrix. However, imparting such characteristics necessitates for ideal interfacial bonding conditions. The presence of a reacted uniform layer of AlN on the surface of a BNNT enhances the interfacial bonding with the metal. While Al is found to have a contact angle of $\sim 140-160{ }^{\circ} \mathrm{C}$, characterizing the poor wettability of the nanotube with the molten metal. Conversely, the interaction between the molten $\mathrm{Al}$ and the $\mathrm{AlN}$ layer is found to result in contact angles between $40-60{ }^{\circ} \mathrm{C}$ (Figure 10 a). The improved wettability between the reacted phase ( $\mathrm{AlN}$ ) as compared to the unreacted $\mathrm{BN}$ surface is a result of the high surface tension $(660 \mathrm{mN} / \mathrm{m})$ providing favorable conditions for wetting ${ }^{55,56}$. Improved wetting conditions between the metal matrix and the nanotube was found to impart increments in elastic modulus of the Al matrix by $2 \times(134 \mathrm{GPa})$, with 
effective transfer of load mechanisms such as crack bridging, nanotube pull-out, etc ${ }^{4,53-55}$ (Figure $10 \mathrm{~b}$ and $\mathrm{c}$ ).
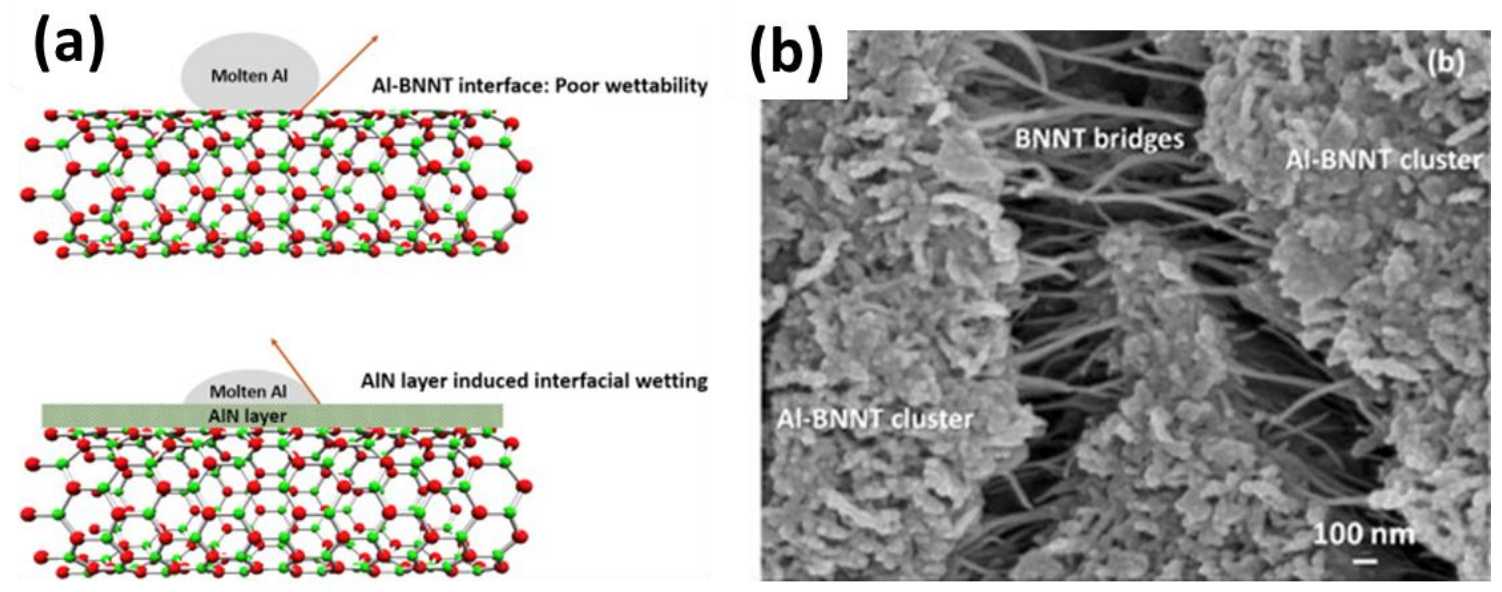

Figure 10 (a) Schematic representation of the interactions between molten $\mathrm{Al}$ and the unreacted BNNT vs. the improved wetting in the presence of an AIN layer. (b) Strengthening mechanism in Al-BNNT in the presence of interfacial reactions, showing BNNTs acting as bridges. (c) ${ }^{4,53-55}$

In addition to the strengthening imparted by strong interfacial interactions between the nanotube and the metal matrix, BNNTs have shown to effectively serve as sites for dislocations to pile up and restrict gliding of the same ${ }^{57,58}$. Dislocation pile-ups along the length of BNNTs were found to increase elastic modulus by up to $16 \%$ with merely 0.045 wt $\%$ BNNT content ${ }^{58}$. This novel strengthening mechanism is often not accounted for by stiffening mathematical models and presents the potential of the nanotubes to reinforce matrices $^{58}$ (Figure 11). 

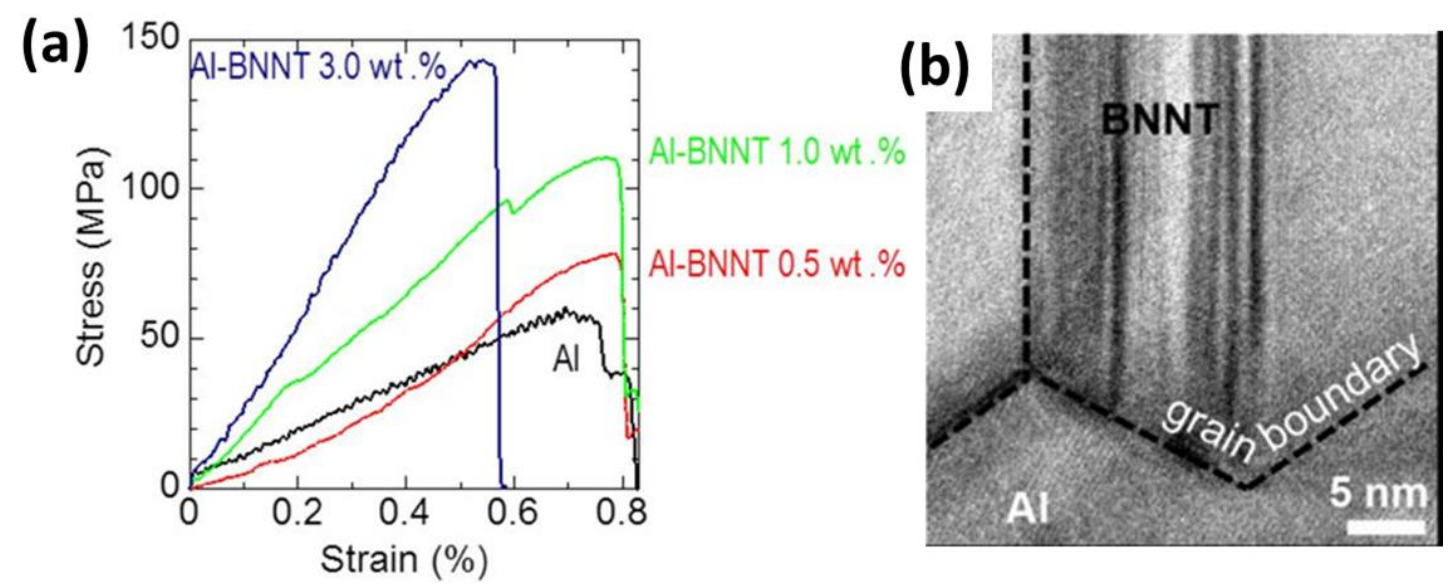

Figure 11 (a) Stress-strain curves of Al-BNNT composite manufactured by the hot rolling process with strengthening mechanisms dominated by (b) BNNTs ability to restrain grain growth and promote the pile-up of dislocations along its length ${ }^{5}$.

\subsection{BNNT reinforced Titanium matrix composites}

Owing to the structural and thermal advantage of Titanium as a metal with high specific strength as compared to other light metals ( $\mathrm{Al}$, and $\mathrm{Mg}$ ), their reinforcement is often sought for in the automotive, aerospace, and naval industries. A common necessity in these applications is their ability to withstand extreme conditions including elevated temperatures, high stresses, prominent oxidation environments.

\subsubsection{Titanium: A suitable metal for extreme environments}

Titanium and its alloys have found applications in applications often experiencing corrosive and thermo-mechanically demanding conditions. Their preference is a result of their high strength to weight ratio (Tensile Strength 950 MPa), their corrosion resistance, and their ability to maintain their mechanical strength even at elevated temperatures (400$500^{\circ} \mathrm{C}$ )(Figure 12). 


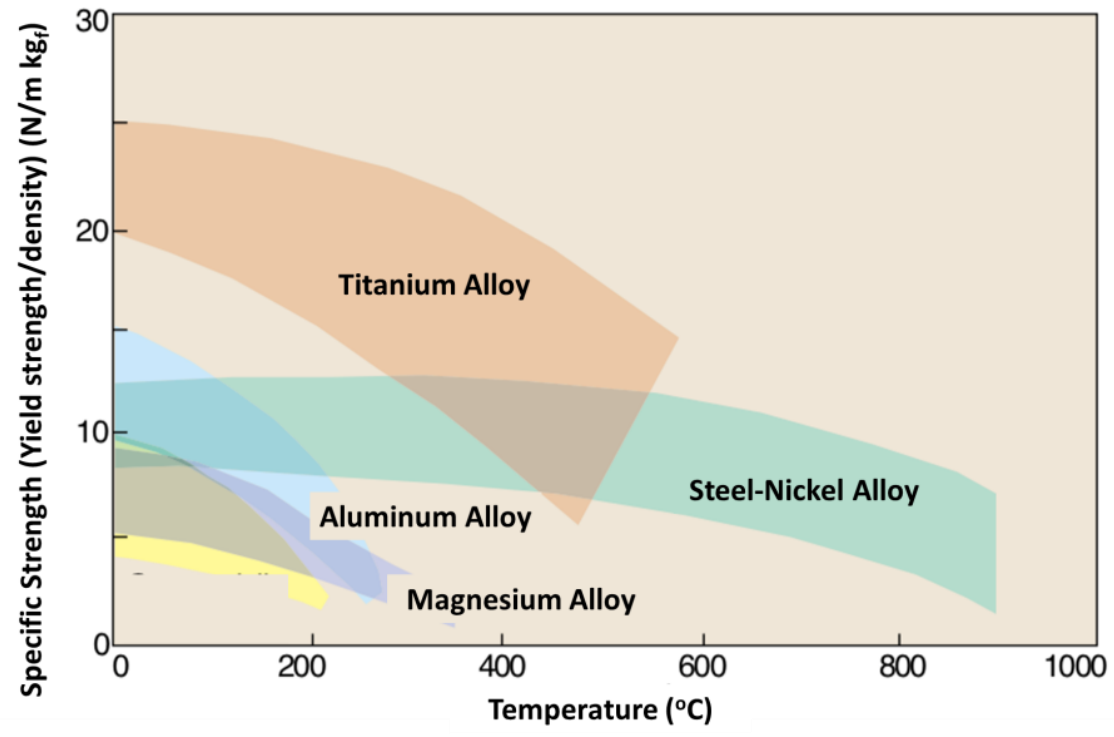

Figure 12 Comparative chart demonstrating the specific strength of multiple structural metals as a function of increasing temperatures ${ }^{12,59-61}$.

While the consumption of Titanium is often found in small quantities across all suitable applications, their use is limited to critical regions experiencing higher mechanical stresses and elevated temperatures. The excellent mechanical characteristics of Titanium can be understood from an atomic perspective. Ti is characterized by having a Hexagonal closed packed (HCP) crystal structure ( $\alpha$-phase) at a temperature below $\sim 882^{\circ} \mathrm{C}$ (Figure 13) ${ }^{12,59-61}$. Upon reaching temperatures higher than $1200^{\circ} \mathrm{C}(\beta$ transus temperature $), \mathrm{Ti}$ experiences phase transformation from a stiff HCP to a ductile body-centered cubic ( $\beta$ phase) ${ }^{12,59-61}$. 
(a)

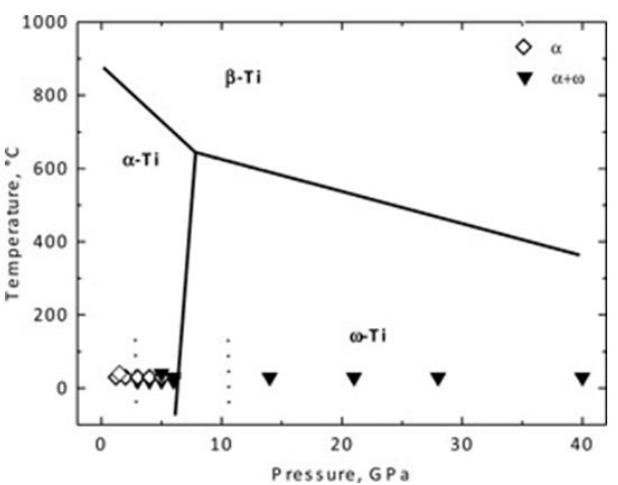

(b)

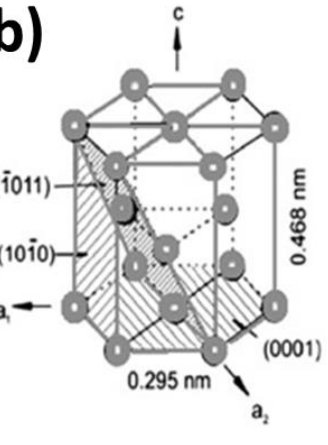

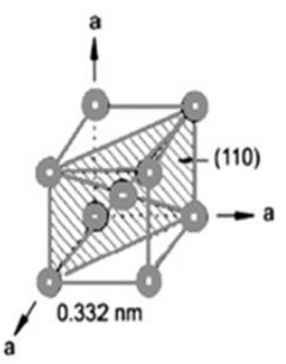

Figure 13 (a) Phase diagram of pure Titanium as a function of increasing pressure and temperature, displaying the presence of three phases: low temperature ( $\alpha$-phase), high temperature ( $\beta$-phase) and metastable $\omega$-phase requiring high pressures. (b) Crystal structures of $\alpha$ - phase Ti showing HCP crystal structure and $\beta$-phase Ti with BCC crystals $^{12,59-61}$.

The versatility of $\mathrm{Ti}$ to tailor its properties by the addition of alloying elements (i.e.,

$\mathrm{Al}, \mathrm{V}, \mathrm{O}, \mathrm{H}$, etc.) has been long investigated. The addition of $\alpha$ - alloying elements such as $\mathrm{O}, \mathrm{Al}, \mathrm{N}, \mathrm{B}$, etc. induces the stiffening of the metal and imparts superior creep resistance. $\alpha$ - alloying involves the diffusion of alloying atoms taking place in the interstices of the $\alpha$ phase ${ }^{12,59-61}$. Thus, resulting in a tightly packed structure with restricted atom movement for deformations. Although, superior strength is desired in the metal, the intrinsic ductility and formability of the BCC $\beta$-phase is also required. The addition of $\beta$-stabilizers such as $\mathrm{V}, \mathrm{Cr}, \mathrm{Fe}$, among others induces a shift in the $\beta$-transus temperature and allowing $\beta$-phase formation at lower temperatures.

It is evident that the presence of both phases in the Ti metal would allow the ideal structural metal capable of combining the stiff nature of the $\alpha$-phase and the ductility of the $\beta$-phase. $\alpha$ - $\beta$ alloys are Ti-based alloys combining $\alpha$ and $\beta$ stabilizers allowing the stability of both phases at lower temperatures (i.e., room temperature) ${ }^{12,59-61}$.Ti6Al4V is the most widely used $\alpha-\beta$ alloy across all applications due to its combined mechanical strength 
(Yield strength: $830 \mathrm{MPa}$, Tensile Strength: $900 \mathrm{MPa}$ ) and ductility ${ }^{12,59-61}$. In which, the contributions from each phase can be modified by subjecting the alloy to thermal processes.

The microstructure of $\alpha-\beta$ alloys is illustrated in Figure 14. In which, primary $\alpha-$ grains are surrounded by $\beta$-phase containing grain boundaries and $\alpha-\beta$ colonies with lamellar morphology. The lamellar morphology of $\alpha-\beta$ colonies is a result of the nucleation and growth of $\alpha$ grains (secondary $\alpha$ ) within the $\beta$-phases.

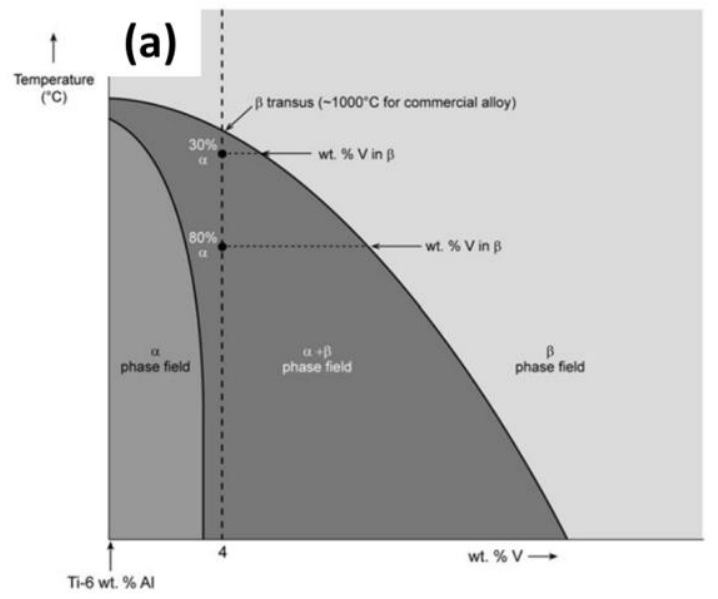

(b)

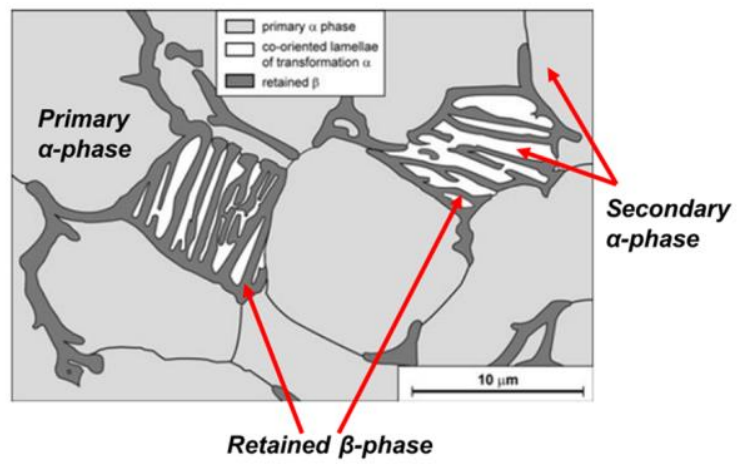

Figure 14 (a) Phase diagram of an $\alpha-\beta$ alloy with $6 \mathrm{wt} . \%$ Al and V as alloying elements, the segmented line represents the phase transformation path of Ti6AI4V alloy ${ }^{59,61}$.

Although Ti6Al4V, exhibits good performance in structural applications and is $50 \%$ lighter than steel, their mechanical performance is far unsuitable for the replacement of many structural steels. Thus, reinforcement of the alloy by nanoparticle addition is observed as a potential alternative.

\section{Suitability of nanoparticle reinforcement in Titanium matrices}

Titanium's high melting point $\sim 1600^{\circ} \mathrm{C}$ requires the processing of the metal in processes involving high temperatures. Common processing routes include casting, sintering, hot forging, and direct powder extrusion. Two significant limitations in the 
reinforcement of Ti matrices by the addition of secondary phases (i.e., $\mathrm{CNTs}, \mathrm{Al}_{2} \mathrm{O}_{3}, \mathrm{SiC}$, $\mathrm{TiB}, \mathrm{TiC}$, etc.) have been identified: (i) thermal expansion of the matrix and/or nanoparticle causing residual stresses. (ii) Thermal stability and reactivity of the nanoparticle with the $\mathrm{Ti}$ at processing temperature (often $>900{ }^{\circ} \mathrm{C}$ ). The high reactivity of the metal with CNTs as reinforcing nanoparticles has been widely reported in literature ${ }^{62-64}$. In which, the rapid precipitation of brittle $\mathrm{TiC}$ occurs at temperatures of $800^{\circ} \mathrm{C}$ during solid-state reaction. Owing to the low thermal stability of CNTs (oxidizes at $\sim 500^{\circ} \mathrm{C}$ ), the microstructures are often dominantly reacted $\mathrm{TiC}$ phases remarkably affecting the mechanical properties of the composite $^{63}$.

\subsubsection{Integrating Boron Nanotubes in a Titanium Matrix}

The thermal stability of BNNTs to up to $900^{\circ} \mathrm{C}$ in air and chemical inertness, pose an advantage to their addition to the Ti-metal matrix. The structural and thermal stability of the nanotubes was evidenced in their addition to $\mathrm{Al}$ matrices, were thin reaction layers

aided in the reinforcement of the composite. Recently, Bhuiyan et al. ${ }^{47,48}$ have pioneered the inclusion of BNNTs to the Titanium matrix. In two separate studies, the authors outline the feasibility of the nanotubes to reinforcing Ti matrices, and the effective reinforcement the BNNTs impart on the composite.

The requirement of a thin reaction layer between the nanotube and the metal matrix was broadly observed in Al-BNNT composites to depend on morphological features of the nanotube and feasibility for reactions. Thus, providing enough interactions to have strong bonding without being detrimental to the nanotube structure. At the atomic level, density functional theory (DFT) calculations, demonstrated the interactions of Titanium atoms 
with BNNTs and B/N vacancy defects potentially present in the nanotubes ${ }^{65}$. Findings of this study showed the strong Ti interactions with BNNTs by displaying high binding energies of $\sim-1.22 \mathrm{eV}$ as compared to $\mathrm{Al}(-0.74 \mathrm{eV})^{65}$. This highlights the importance of defect-free BNNTs to achieve moderate interactions between the Ti and the nanotubes. Thus, nanotubes with smooth morphology obtained by high-pressure vapor/condenser method are desirable and will be used in this study.

The thermodynamically feasible reactions and transformations experienced between Ti and BN phases are identified in Figure 15 at processing temperatures $>1000^{\circ} \mathrm{C}^{11,66}$ :

$\mathrm{Ti}+\mathrm{BN}=\mathrm{TiB}_{2}+\mathrm{TiN}$

$\mathrm{Ti}+\mathrm{TiB}_{2}=\mathrm{TiB}$

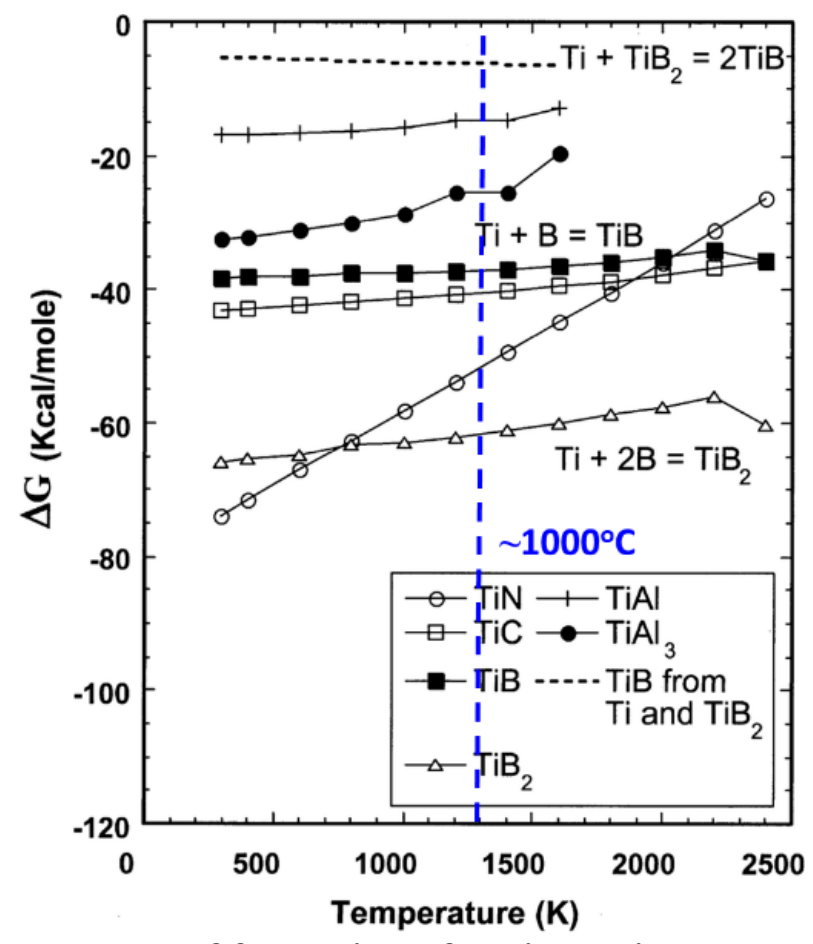

Figure 15 Gibb's free energy of formation of various Ti and $\mathrm{BN}$ reactions ${ }^{11,66}$. 
Previous studies have explored the thermodynamic feasibility of reactions, including that of $\mathrm{TiB}_{2}$ with the lowest Gibbs free energy of formation $(\Delta \mathrm{G}=-62 \mathrm{kcal} / \mathrm{mole})^{66}$. Bhuiyan et al. ${ }^{47,48}$ described the formation of $\mathrm{TiB}_{2}$ nanoparticles on the surface of BNNTs after exposing the composite powder to $800^{\circ}$ for a period of $30 \mathrm{~min}^{48}$. The $\mathrm{TiB}_{2}$ reaction is expected to occur at the direct interface between the $\mathrm{Ti}$ and the nanotube due to the higher localized $\mathrm{B}$ concentration. The reaction is driven by the diffusion of released $\mathrm{N}$ atoms into Ti forming a solid solution in the matrix.

Although TiB formation is a less thermodynamically favorable reaction, the kinetics governing the diffusion of boron in $\mathrm{TiB}_{2}$ and the growth of $\mathrm{TiB}$ results in the presence of TiB whiskers. After exposure of the Ti-coated nanotubes to $900^{\circ} \mathrm{C}$ for 1 hour, $\mathrm{TiB}_{2}$ is shown to grow radially into the hollow nanotube producing elongated plate-like structures $^{48}$. The decreased localized B concentration and thermal energy induce the formation of $\mathrm{TiB}$ phases in the form of whiskers, as describe by equation 2 . Thus, observing the $\mathrm{TiB}_{2}$ phase as a transition phase for the formation of $\mathrm{TiB}$ given the $\mathrm{B}$ concentration in the reaction interface to be between 18 to $18.5 \%$. Such findings serve as a guideline in the selection of processing conditions to control the reactions between Ti-matrix and BNNTs. 

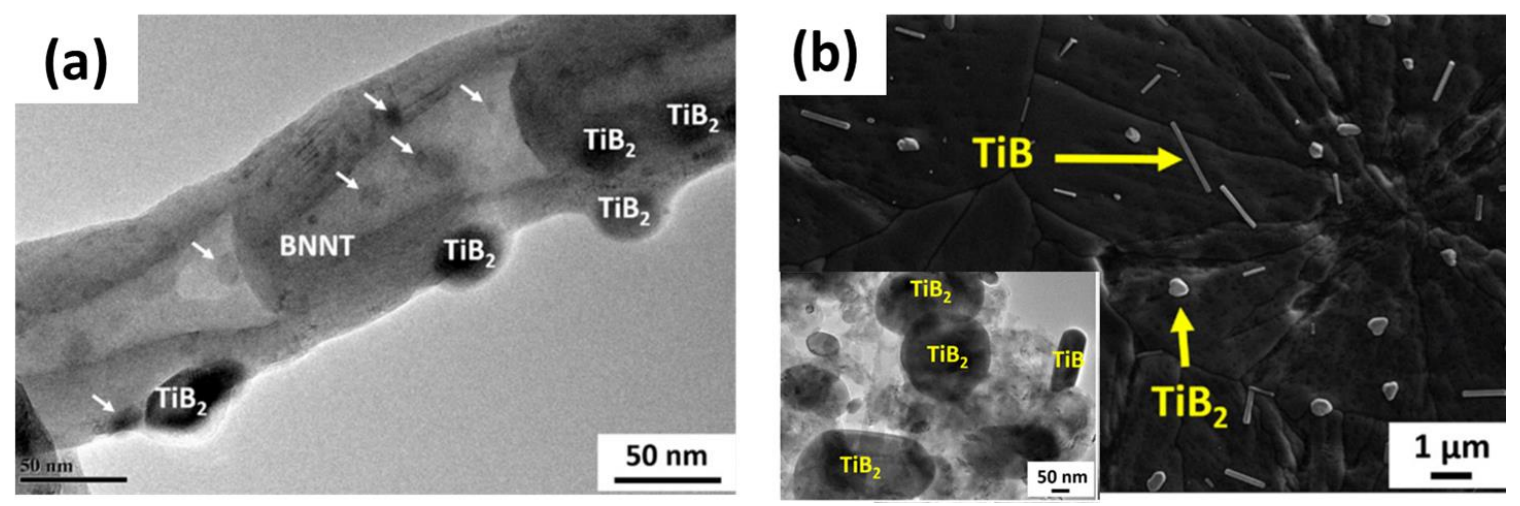

Figure 16 (a)TEM micrograph showing $\mathrm{TiB}_{2}$ reaction phases at the surface of a single nanotube coated $\mathrm{Ti}$ and exposed to $8^{\circ} 0^{\circ} \mathrm{C}$ for $30 \mathrm{~min}$. (b)Formation of $\mathrm{TiB}_{2}$ nanoparticles and TiB needle/whiskers after exposure of Ti-coated nanotubes to $900^{\circ} \mathrm{C}$ for 1 hour $^{48}$.

\section{Improved mechanical performance}

To the author's knowledge, a single study has evaluated the mechanical properties imparted by the BNNTs in reinforcing a Titanium matrix ${ }^{47}$. In which, conventional sintering of a Ti-BNNT ( 4 vol. $\%$ ) composite powder was performed at temperatures of $800-100^{\circ} \mathrm{C}$ for $1-3$ hours. The BNNTs with bamboo-like morphology used by Bhuiyan et al. ${ }^{47}$ demonstrated to be stable to temperatures of $800^{\circ} \mathrm{C}$ and heating periods of 1 hour by retaining their conical structure and tubular structure. Strengthening of the Ti matrix was observed to up to $45 \%$ (984 MPa) as compared the pure Ti sintered at similar conditions $(678 \mathrm{MPa})^{34}$. The strengthening was mainly attributed to the ability of BNNTs to restrain dislocation movement with nanotubes effectively bearing larger stresses. Moreover, sintering of the composites at $1000^{\circ} \mathrm{C}$ for 3 hours was found to result in the complete transformation of BNNTs to sharp and brittle TiB phases (Figure 17) ${ }^{34}$. 

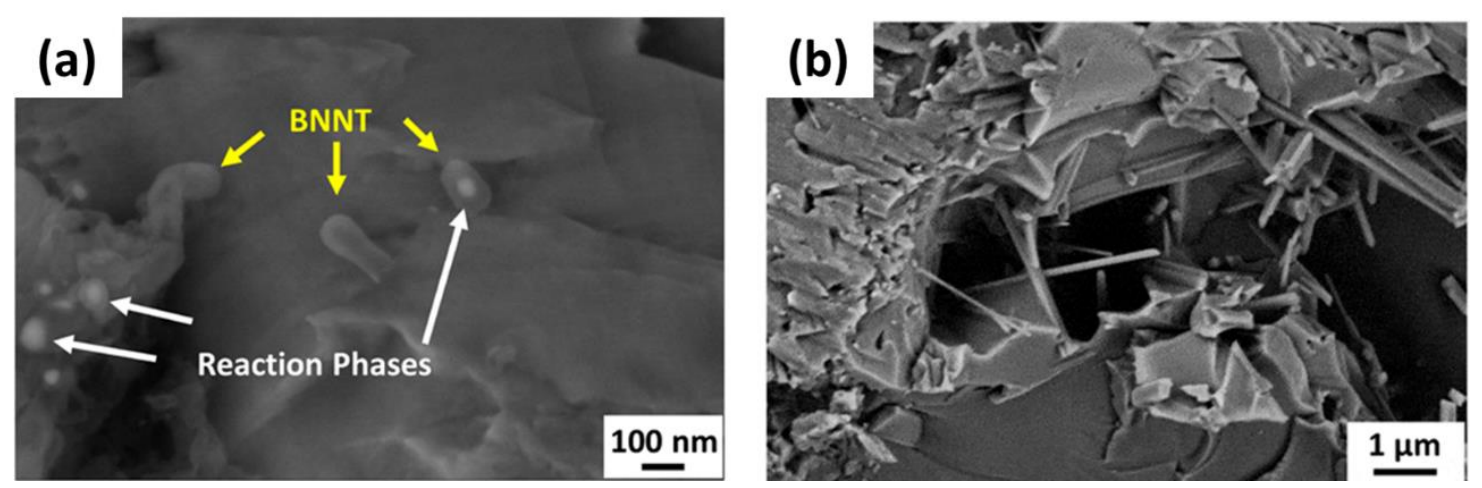

Figure 17 Fracture surface of Ti-BNNT composite (a) sintered at $800^{\circ} \mathrm{C}$ for $1 \mathrm{~h}$ after experiencing compressive fracture showing $\mathrm{TiB}_{2}$ as primary reaction phases and survived BNNTs, (b) composite sintered at $1000^{\circ} \mathrm{C}$ for $3 \mathrm{~h}$ showing abundant TiB whiskers as a result of $\mathrm{Ti}-\mathrm{BNNT}$ reactions ${ }^{34}$.

\subsubsection{Current challenges:}

It is clear from the previous efforts ${ }^{32,47,48,50,55,57,67}$ that the addition of BNNTs to the Titanium matrix hold great potential as structural materials with significant increments in mechanical properties. The studies have been limited by the use of nanotubes synthesized via ball milling and annealing processes rendering them with bamboo-like morphologies. Thereby having intrinsic defect sites favorable for the nucleation and growth of undesirable reaction products.

Therefore, the main objective of this thesis is to synthesize Titanium reinforced BNNT composites by implementing long $(100-200 \mu \mathrm{m})$ BNNTs. The surface characteristics (smooth) of the BNNTs used in this study are expected to restrict the evolution of interfacial reactions as compared to bamboo-shaped BNNTs used by earlier researchers ${ }^{46}$. Bhuiyan et al. concludes that the reactivity of BNNTs with the Ti matrix can be addressed by limiting the kinetics ${ }^{46}$. Hence, Spark Plasma Sintering as a fast consolidation technique is used to impart short consolidation periods $(10-30 \mathrm{~min})$ that will minimize formation of $\mathrm{TiB}_{2}, \mathrm{TiB}$, and $\mathrm{TiN}$ phases. 
Powder metallurgy routes such as SPS are techniques which require an initial feedstock material in the form of a powder. The homogenous dispersion of BNNTs has been previously attained by ball milling processes, and solvent assisted dispersion routes $^{44,47,54,68}$. The first is known to introduce contaminants and potential defects in the tubular structure. While the dispersion of BNNTs in solvents has been implemented in prior studies, the distinct length $(100-200 \mu \mathrm{m})$ of nanotubes introduced in this study present an added entanglement to the high surface energy BNNTs. Two dispersion techniques will be implemented in the dispersion of BNNTs to determine their suitability to the SPS processing of the composite:

(1) Dispersion by wet chemistry route: Ionic and non-ionic surfactants have shown potential in reducing the surface tension between the nanotubes and the aqueous dispersion media ${ }^{69-71}$.

(2) High-energy induced dispersion: The use of tip sonication has shown the effective dispersion of reinforcing nanoparticles 1D and 2D structures (i.e. CNTs, Graphene nanoplatelets, BN sheets, etc.). The high energy vibrations induced by the sonication are capable of breaking agglomerates and rendering composites with uniform dispersions ${ }^{54,72-74}$. 


\section{CHAPTER III: MATERIALS \& EXPERIMENTAL METHODS}

This chapter outlines the materials and experimental procedures involved in the dispersion of BNNTs by wet chemistry and high energy route in the synthesis of Boron Nitride Nanotube-reinforced Titanium composites. The experimental details to evaluate the microstructural and mechanical properties of the synthesized composite are also outlined.

\section{Materials:}

\subsection{Boron Nitride Nanotubes}

Boron Nitride Nanotubes (BNNTs) synthesized via a pressurized vapor/condenser method (PVD) were obtained from BNNT, LLC (Newport News, VA). The nanotubes were obtained in a purified state with $<1 \% \mathrm{BN}$ impurities in the form of fibril balls (Figure $18)^{75}$

(a)

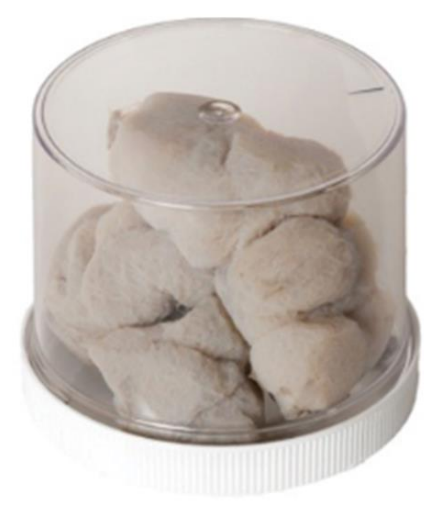

(b)

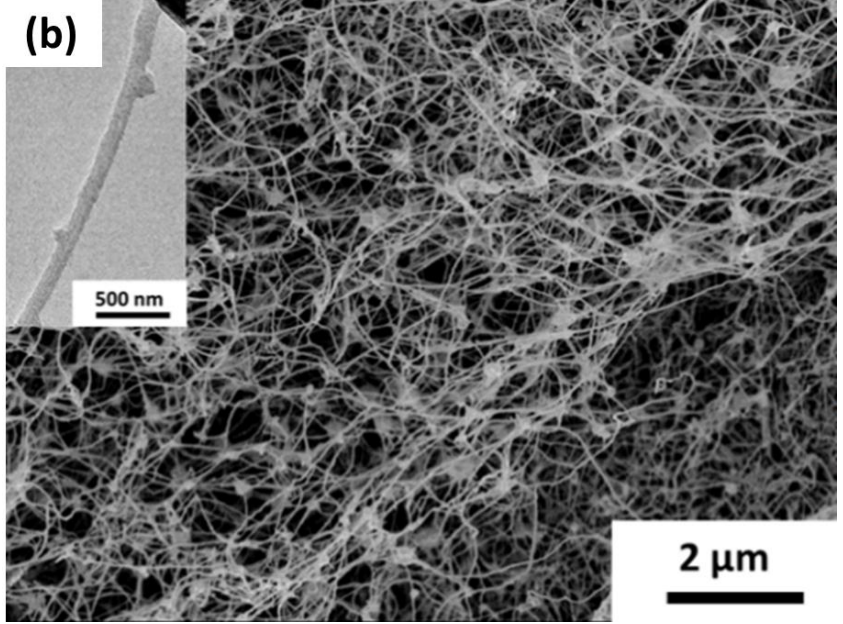

Figure 18 As received (a) BNNT fibril balls synthesized by PVC method $^{75}$, (b) SEM micrograph of entangled long and ultrafine BNNTs. Inset shows the smooth morphology of a single nanotube. 
Purified BNNTs with the absence of catalyst impurities and minimal BN nanoparticles are desirable in the manufacturing of composites undergoing hightemperature processes due to the high reactivity of impurities relative to the BNNTs. The acquired BNNTs are characterized by having long $(100-200 \mu \mathrm{m})$ and ultra-fine $(5-10 \mathrm{~nm})$ morphologies with typically 1-5 walls ${ }^{75}$. The strengthening of fiber-reinforced composites has been theoretically modeled by the shear lag model $^{4,53}$.

$l_{c}=\frac{\sigma_{f} d}{\sigma_{m}}$

The model assumes strengthening in the composite is a function of the shear generated between the fiber $\left(\boldsymbol{\sigma}_{\mathrm{f}}\right)$ and the matrix $\left(\boldsymbol{\sigma}_{\mathrm{m}}\right)$. In which, effective load transfer between the matrix to reinforcing particles occurs if the reinforcing fibers exceed a critical length $\left(l_{c}\right)$, generating maximum shearing stress between the fiber and the matrix. Therefore, it is expected that the long BNNTs used in this study will render composites with enhanced mechanical strength

\subsection{Titanium Alloy: Ti6Al4V}

A commercially available Titanium alloy (Ti6Al4V) with principal elements in the composition of 6 wt.\% Al, and 4wt.\% V was obtained from TEKNA, Sherbrooke, Canada. Ti6Al4V is a two-phase $(\alpha-\beta)$ Titanium alloy with balanced strength and ductility widely used in the aerospace, marine, and automotive industries. The Ti6Al4V powder used in this study is of spherical morphology with an average particle size of $8.25 \pm 4 \mu \mathrm{m}$ and 4.5 $\mathrm{g} / \mathrm{cm}^{3}$ density Figure 19. 


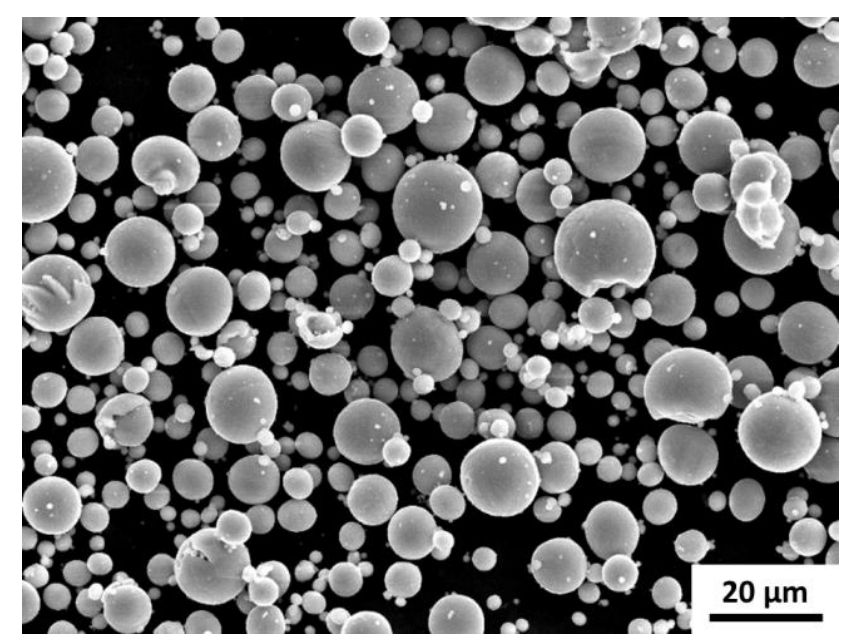

Figure 19 As-received Ti6Al4V with spherical morphology and average particle size of $88.25 \pm 4 \mu \mathrm{m}$

Table 3 elemental composition of the as-received Ti6Al4V powder obtained by Energy Dispersive Spectroscopy (EDS) analysis. The absence of Oxygen in the table is evidence to the extremely low content of $\mathrm{O}_{2}$ in the powder during its processing incapable of being detected by the instrument. The high affinity of Ti6Al4V to Oxygen is known to result in the rapid oxidation of the powder if exposed to $\mathrm{O}_{2}$ rich environments. Limiting the $\mathrm{O}_{2}$ intake of the powder is of high importance to the densification by sintering, this was achieved by maintaining the powder in a protective Ar environment.

Table 3 Elemental composition of as-received Ti6Al4V powder obtained from EDS analysis

\begin{tabular}{ccccc}
\hline Element & Al $(\mathbf{K})$ & Ti $(\mathbf{K})$ & V $(\mathbf{K})$ & Fe $(\mathbf{K})$ \\
\hline \hline Atom \% & $8.4 \pm 0.8$ & $87.6 \pm 2.4$ & $3.3 \pm 0.8$ & $0.7 \pm 0.4$ \\
\hline
\end{tabular}

\section{Synthesis of Ti-BNNT composite}

The synthesis of the BNNT reinforced $\mathrm{Ti}$ matrix composites entailed the homogeneous dispersion of BNNTs in an aqueous media and subsequent attachment to 
Ti6Al4V powder particles. The composite powder was finally consolidated by powder metallurgy route.

\subsection{Dispersion techniques}

Two distinct dispersion routes were used in the dispersion of BNNTs to evaluate their suitability to the Spark Plasma Sintering processing and their ability to produce a homogenous distribution of nanotubes over the Ti matrix. A wet chemistry method and high energy induced tip sonication were introduced to the entangled and agglomerated nanotubes.

\subsubsection{Dispersion of BNNT by wet chemistry route}

The hydrophobic nature of BNNTs poses a challenge to their homogenous dispersion in the aqueous medium. In this study, a non-ionic surfactant (Pluronic) is introduced to assist in the dispersion of the high surface energy nanotubes. Non-ionic surfactants have shown to be the most effective surfactants to allow the effective dispersion of nanotubes, yielding high concentrations of individual BNNTs ${ }^{55,76}$. The amphiphilic nature of surfactants allows them to possess both hydrophobic and hydrophilic properties. In their introduction to a nanotube-aqueous mixture, they reduce the surface tension between BNNTs and aqueous media by their adsorption to the nanotubes. This process entails the adherence of surfactant molecules containing apolar (hydrophobic) tails to the hydrophobic surface of the nanotubes, leaving the polar (hydrophilic) molecules to interact with the aqueous medium $55,69,74$. Their advantage to other surface functionalization techniques is their ability to stabilize BNNTs in an aqueous medium without disturbing the $\mathrm{sp}^{2}$ hybridization of the BNNTs ${ }^{76}$. The surfactant-assisted dispersion was carried out in a 
DI water suspension. The non-ionic surfactant (Pluronic) was introduced to the BNNT/DI water mixture in a 1:3 BNNT to surfactant ratio. The higher concentration of Pluronic over BNNTs is desirable to allow the solution to reach the critical micelle concentration in which surface tension reduction process occurs. The aqueous mixture (BNNTs/Pluronic/DI water) was bath sonicated for a period of $72-96$ hours to promote the dissolution of surfactant molecules in the aqueous media and allow the adherence to the nanotubes. The addition of surfactant, nanotubes, and DI water was performed continuously throughout the dispersion process. The dispersion was considered as complete when no evident agglomerates were observed. The final concentration of BNNTs in the solution was of 10 $\mathrm{mg} / \mathrm{ml}$ of DI water.

Following the stable dispersion of BNNTs, Ti6Al4V powder was added to the BNNT solution to obtain a $1 \mathrm{wt} . \%$ BNNT concentration. To promote the adherence of BNNT onto the Ti powder particles, the solution was magnetically stirred for a period 15 min. The final solution was subsequently oven dried under vacuum conditions at $70^{\circ} \mathrm{C}$ to minimize powder oxidation. The composite powder was subjected to final heating at $200^{\circ} \mathrm{C}$ for 2 hours to evaporate residual surfactant in the powder.

\subsubsection{High energy induced dispersion by tip sonication}

Ultrasonication has been a reliable method for the dispersion and exfoliation of

CNTs and other nanoparticles ${ }^{73,77-79}$. The imparted high energy cavitation is capable of producing strong shear forces to disentangle and disperse agglomerates. Although the high energy introduced during ultrasonic cavitation, careful consideration of sonication parameters should be taken. Exceeding sonication limits could lead to the introduction of 
surface defects on the nanotubes and increase their reactivity during thermal processing ${ }^{73,77-79}$. The success in the dispersion of BNNTs by high energy induced sonication is highly dependent on ${ }^{73,77-79}$ :

(i) The properties governing the solvent (i.e., vapor pressure, viscosity, interactions with the nanoparticles); maximized dispersion by cavitation induced dispersions were found to occur in solvents with vapor pressures $<10 \mathrm{~mm} \mathrm{Hg}$ due to the high energy release

(ii) Intensity of sonication; higher sonication intensity is bound to produce higher acoustic amplitudes and thus produce collapsing of bubbles with higher force

(iii)Time of sonication; increasing time of sonication will result in higher energy input into the dispersion.

This study introduces ultrasonic cavitations in the dispersion of high surface energy BNNTs. A tip sonication system Vibra-Cell VCX750 (Sonic \& Material, Inc., Newtown, CT) with $19 \mathrm{kHz}$ frequency, $750 \mathrm{~W}$ power, and a $3 / 4$ high gain sonotrode were used. The dispersion was carried out in acetone solvent under 70\% amplitudes $(\sim 47.2 \mu \mathrm{m})$ and a period of $45 \mathrm{~min}$. Due to the high energy released in the form of heat during the sonication process, the solution was kept enclosed by an ice bath to prevent significant temperature changes. The final dispersion had a BNNT concentration of $0.09 \mathrm{mg} / \mathrm{ml}$ of acetone. The solution was subsequently bath sonicated for 1 hour to maximize the stability and dispersion of nanotubes in the solution.

Finally, Titanium powder was introduced in the solution (1 wt. \% BNNT) and magnetically stirred for 1 hour to promote the adherence of BNNTs on the surface of 
powder particles. The solution was then oven dried at $50^{\circ} \mathrm{C}$ in vacuum conditions to minimize the oxidation of the composite powder.

\subsection{Pressure-assisted powder metallurgy}

Consolidation of the dispersed BNNTs in Ti-matrix was performed by Spark Plasma Sintering (SPS) in a Thermal Technologies model 10-4 spark plasma sintering apparatus (Thermal Technologies, LLC, Santa Rosa, CA). The rapid densification allowed during the SPS process is attributed to the simultaneous applied pressure and heat to result in the sintering of the powder. SPS presents advantages to the processing of Ti-BNNT composites due to their short sintering times (10-30 min), limiting the kinetics of interfacial reactions. Thus, the synthesis of Ti-BNNT composites with minimal interfacial reactions (Ti/BNNT) and grain growth is expected. To address the thermally activated reaction challenge between the BNNT and Titanium, two sintering temperature schedules were investigated. Sintering of Ti-BNNT composites was evaluated at both (1) low (600 $\left.750^{\circ} \mathrm{C}\right)$ and $(2)$ high $\left(950^{\circ} \mathrm{C}\right)$ temperature regimes to evaluate their suitability in the processing of Ti-BNNT composites.

\subsubsection{Low-temperature sintering}

Systematic experimentation was performed to identify optimum sintering conditions that would allow the densification of pure Ti6Al4V and ideally the consolidation of Ti-BNNT composites. The yielding of elevated temperatures to sinter Titanium was traded by the introduction of high pressures (>300 MPa) to induce sufficient plastic deformation and promote sintering mechanisms to evolve. 
Table 1 describes the parameters under study to obtain a near fully dense composite. Owing to the elevated pressures involved, a $15 \mathrm{~mm}$ Tungsten Carbide (WC) die was used as opposed to the common graphite. Graphite foil was used to prevent fusion and interactions between the die and the sintered powder in addition to facilitating the removal process.

Table 4 Sintering parameters used for low-temperature sintering of Ti6Al4V

\begin{tabular}{cccc}
\hline $\begin{array}{c}\text { Temperature } \\
\left({ }^{\circ} \mathbf{C}\right)\end{array}$ & $\begin{array}{c}\text { Pressure } \\
(\mathbf{M P a})\end{array}$ & $\begin{array}{c}\text { Heating rate } \\
\left({ }^{\circ} \mathbf{C} / \mathbf{m i n}\right)\end{array}$ & $\begin{array}{c}\text { Hold Time } \\
(\mathbf{m i n})\end{array}$ \\
\hline 600 & 300 & 50 & 10 \\
\hline 600 & 500 & 50 & 10 \\
\hline 600 & 555 & 50 & 10 \\
\hline 750 & 555 & 100 & 10 \\
\hline
\end{tabular}

\subsubsection{High-temperature sintering}

Sintering of pure Ti6Al4V and Ti-BNNT composites was performed at temperatures of $950^{\circ} \mathrm{C}$, pressures of $60 \mathrm{MPa}$ and $100^{\circ} \mathrm{C} / \mathrm{min}$ heating rates. Sintering was performed in periods of $10 \mathrm{~min}$ to minimize interfacial reactions between the nanotube and the Ti-matrix. Sintering conditions were chosen by considering temperatures below the $\beta$ transus temperature of Ti6Al4V which occurs at $\sim 1000^{\circ} \mathrm{C}$. Maintaining sintering temperature below the $\beta$-transus reduces the residual $\beta$-phase in the composite and results in a well-balanced strong yet ductile alloy. Sintering was performed using a $20 \mathrm{~mm}$ graphite die and graphite foil lining the powder and punches for ease of pellet removal. 
The continuous displacement of the sintering punches in contact with the sintered powder was recorded and analyzed by determining the corresponding instantaneous relative density ( $\left.\rho_{\text {instantaneous }}\right)$ of the samples:

$\rho_{\text {instantaneous }}=\left(\frac{L_{f}}{L}\right) \rho_{f}$

In which, $L$ corresponds to the thickness of the sample throughout the SPS process and is time dependent. Similarly, $L_{f}$ and $\rho_{f}$ are the final thickness of the sample and the final densification achieved. The instantaneous relative density curve as a function of time is known to provide insights on the mechanism of densification occurring throughout the SPS process.

\section{Microstructural Characterization}

The graphite foil was removed from the as-sintered Ti and Ti-BNNT samples by using $\mathrm{SiC}$ grinding paper. Densification of the sintered specimens was evaluated by the apparent density of the specimen obtained via Archimedes density method:

$\rho_{\text {relative }}=\frac{m_{\text {air }}}{\left(m_{\text {air }}-m_{\text {water }}\right)}$

The relationship (4) relates the mass of the sintered specimen in air conditions $\left(m_{\text {air }}\right)$ and is inversely proportional to the difference in mass of the pellet in air and water $\left(m_{\text {water }}\right)$. The apparent density of the as-sintered specimens was compared to the theoretical density of Ti6Al4V $\left(4.5 \mathrm{~g} / \mathrm{cm}^{3}\right)$ and that of a Ti-BNNT composite with $1 \mathrm{wt} \%$ BNNT. The theoretical density of the Ti-BNNT composite was estimated by implementing the general rule of mixtures ${ }^{80}$ : 
$\rho_{T i-B N N T}=\rho_{B N N T} * V_{B N N T}+\rho_{T i} * V_{T i}$

In which, the $\rho_{B N N T}$, and $V_{B N N T}$ correspond to the density $\left(1.4 \mathrm{~g} / \mathrm{cm}^{3}\right)$ and volume fraction of the BNNT $(\sim 0.031)$ in the composite and $\rho_{T i}\left(4.5 \mathrm{~g} / \mathrm{cm}^{3}\right), V_{T i}(0.968)$ to those of the Ti6Al4V. Rule of mixtures is a widely accepted mathematical relationship that allows the estimation of mechanical characteristics of composites. The relationship is based under the assumption that the effective mechanical properties of a composite are a weighed contribution by the filler and matrix. Although, not implemented in this study for the prediction of mechanical properties, its derivation was used to estimate the theoretical density of the composite with $1 \mathrm{wt} . \%$ BNNT as $4.40 \mathrm{~g} / \mathrm{cm}^{3}$.

\subsection{X-ray Diffraction: Identification of phases in consolidated Ti-BNNT composites}

Controlling the resulting interfacial phases during sintering and post-processing are of outmost importance, as it will directly affect the mechanical characteristics of the composite. High strength interphase between nanotube/metallic matrix has shown to be beneficial in the efficient transfer of loads. Thus, identification of phases present in the Ti and Ti-BNNT specimens after sintering was performed by X-ray Diffraction (XRD) at a scan rate of $2 \%$ min using $\mathrm{Cu} \mathrm{K} \alpha$ radiation $(\lambda=1.542 \AA)$. A Bruker D5000 X-ray diffractometer (Billerica, MA) was used to obtain diffraction patterns at an operating voltage of $40 \mathrm{kV}$ and $35 \mathrm{~mA}$ current.

Owing to the high pressures involved in low-temperature sintering, analysis of the obtained patterns was performed to identify changes in the crystal structure. Bragg's Law (Eq. 7)was used to obtain d-spacing of at least 6 peaks in the diffraction pattern, and 
geometrical relationships of an HCP crystal (Eq. 8) were used to obtain lattice parameters $(a, c)$.

$\lambda=2 d \sin (\theta)$

$\frac{1}{d^{2}}=\frac{4}{3}\left(\frac{h^{2}+h k+k^{2}}{a^{2}}\right)+\frac{l^{2}}{c^{2}}$

In which, $\lambda$ corresponds to the wavelength of the $\mathrm{x}$-ray source, $\theta$ is the diffraction angle, and $(\mathrm{h}, \mathrm{k}, \mathrm{l})$ represent the Miller indices of the planes reflected in the diffraction pattern. Such analysis would allow us to investigate potential residual strains introduced during the high pressure assisted sintering of Ti and Ti-BNNT at low temperatures.

\subsection{Metallography: Microstructural evolution as a function BNNT addition}

Microstructural characterization was performed to evaluate the effect of BNNTs on the grain size, and $\beta$-phase distribution of the composite as a function of sintering conditions. Reinforcement of metal matrices by reduction of grain size is a wellestablished mechanism. The introduction of secondary phases can lead to the restriction of grain growth.

The pure $\mathrm{Ti}$ and $\mathrm{Ti}-\mathrm{BNNT}$ samples were prepared following metallographic standards to minimize surface roughness and remove the surface imperfection. Samples were ground using $\mathrm{SiC}$ paper up to 1200 grit and polished using $\mathrm{Al}_{2} \mathrm{O}_{3}$ media with up to $0.5 \mu \mathrm{m}$ particle size. The chemical etching was performed to the polished surfaces to reveal microstructural features including grains, pores, etc. Kroll's reagent with main components as DI water, fluoric acid and nitric acid in a 100:3:6 ratio respectively. Characterization of the etched surfaces was evaluated using a ZEISS Axioscope (Jena, Germany) optical 
microscope and ImageJ (NIH) software was implemented for grain size measurements. At least 40 - 50 grain measurements were obtained per sample to obtain significant statistical data.

\subsection{Electron microscopy: Morphology of interfacial interactions of BNNT/metal}

Field Emission Scanning Electron Microscopy (FE-SEM) JEOL JSM-6330

(Peabody, MA) was used in the evaluation of the fracture surface of ti and Ti-BNNT composites. Given the thermodynamically feasible reactions in the Ti-BN system to include $\mathrm{TiB}, \mathrm{TiB}_{2}$, and $\mathrm{TiN}$. For which the precipitation of $\mathrm{TiB}_{2}$ phases result in irregularly shaped nanoparticles, and TiB phases are portrayed as sharp needle structures. The morphology of potential reactions present at the interface of the BNNT/metal was imaged to serve as evidence of their presence or lack thereof. Energy Dispersive Spectroscopy (EDS) was implemented to identify the elemental distribution of phases present in the composite.

Transmission Electron Microscopy (TEM) was performed in the Ti-BNNT specimens to obtain an understanding of the reaction phases interacting at the interface between the nanotube and the metal. Electron transparent specimens were prepared via Focused Ion Beam machining (dual beam JEOL JIB-4500 focused ion beam/SEM, Tokyo, Japan). Assessment of bonding mechanisms and reactions during the processing were also evaluated by conventional TEM (FEI/Philips CM-200T, Hillsboro, OR) and highresolution scanning TEM (TECNAI F20, Thermo Scientific, MA) energy dispersive X-ray spectroscopy (EDX) maps of the specimens. 


\section{Mechanical Characterization: Strengthening induced by BNNTs}

The strengthening observed in BNNT reinforced metal composites is highly dependent on the interfaces between the nanotubes and the metal matrix. This has led to stress transfer mechanisms evidenced by pull-out of BNNTs from the matrix. The mechanical performance of the composites at multiple length scales was probed by indentation and compression techniques. Evaluating the deformation mechanisms in the composite will be performed by optical and scanning electron microscopy to obtain an understanding of the hierarchical stress-transfer and strengthening mechanisms in the TiBNNT composite.

In-situ and ex-situ nanoindentation techniques were used to probe the localized mechanical characteristics of the composite as a function of microstructural distributions. Depth-sensing nanoindentation by a Triboindenter (TI 900) (Hysitron Inc., Minnesota, $\mathrm{MN}$ ), equipped with a diamond Berkovich tip (100 nm radius) was used to performed 25 indents per region of interest. Quasi-static indentation with a maximum load of $8500 \mu \mathrm{N}$ was introduced with a 5 s hold to minimize creep effects and avoid nosing effects in the curve. The mechanical characteristics in the presence of BNNTs as compared to its matrix were evaluated, and the Elastic modulus $(\mathrm{E})$ and Nanohardness $(\mathrm{H})$ were obtained. Oliver Pharr method was used in the analysis of load-displacement curves. In which, E values serve as an indirect measure of the matrix-nanotube bond strength, and nanohardness increments display the ability of nanotubes to pin dislocations. Also, in-situ mechanical investigations were carried out inside the SEM by implementing a Picoindenter (Hysitron PI 87, Minnesota, MN). A sharp cube corner tip (40 $\mathrm{nm}$ radius) was used in to induce 
maximum localized stresses that would allow visualizing BNNT related strengthening mechanisms. Quasi-static indentation loading was performed in displacement control with maximum displacements of 1-10 $\mu \mathrm{m}$ and experienced deformations were captured in-real time for further analysis. Up to 10 indentations per region were performed for statistical analysis.

Vickers' microhardness was used to evaluate the resistance to plastic deformations at the micro-scale length. In which, larger areas of interaction between the probe and the specimen will display collective reinforcement of nanotubes, interfaces, and matrix. Microhardness was performed using a LECO LM910AT (Saint Joseph, MI) tester with a diamond Vickers indenter. Loads of $300 \mathrm{gf}$ and dwell time of 10 seconds were used to make at least 10 indents.

To evaluate the bulk mechanical properties of the Ti-BNNT composite, macroscale compression experiments were performed. Wire EDM machined cylinders with 2 mm diameter and 3-4 mm height out of the sintered pellets were used as the test samples (Figure 20). Four samples for each studied condition were compressed using an MTI SEMtester (Albany, USA) with a $4000 \mathrm{~N}$ capacity load cell at compression rates of 500 $\mu \mathrm{m} / \mathrm{min}$. 


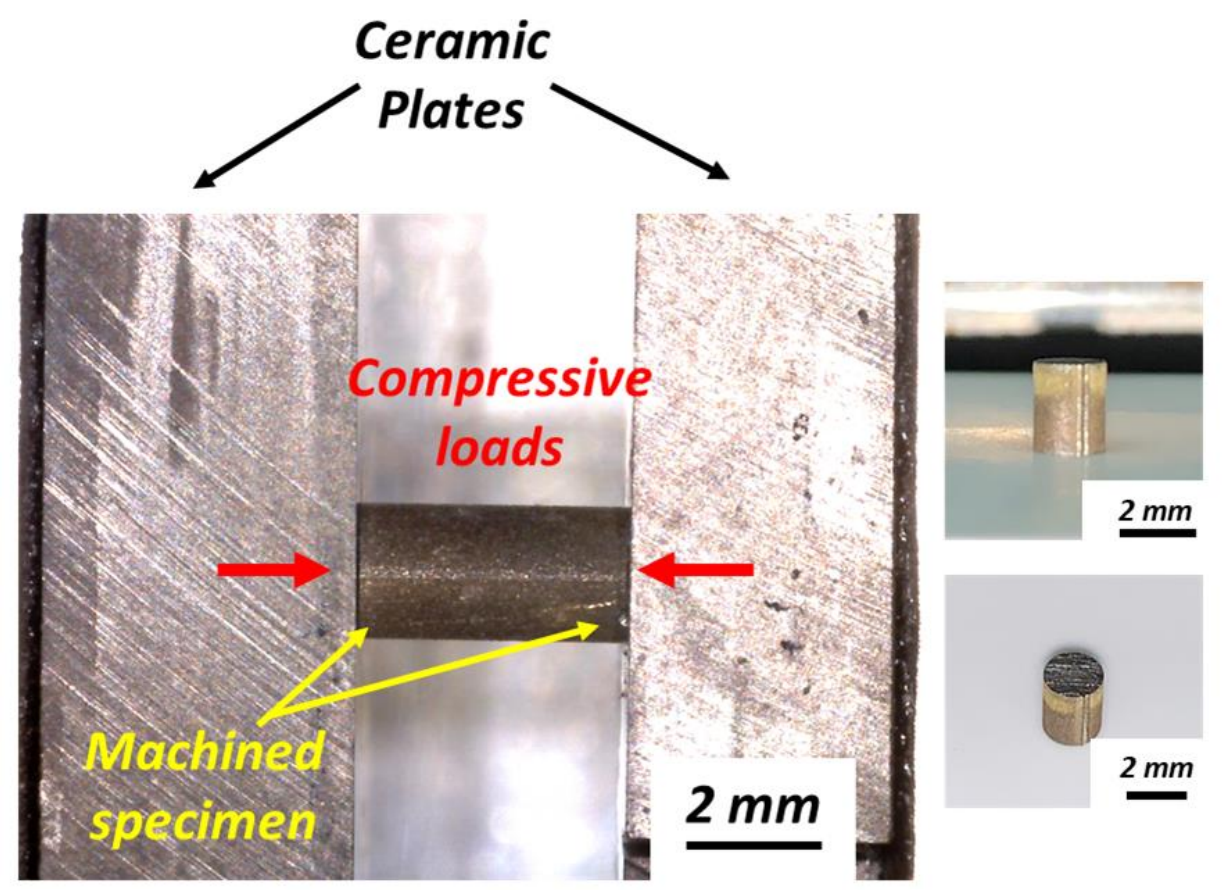

Figure 20 Experimental set-up used to evaluate the bulk compressive properties 


\section{CHAPTER IV: RESULTS AND DISCUSSION}

This chapter describes the experimentally obtained results, followed by a detailed discussion in the synthesis of the composites, reacted phases role and the strengthening mechanisms at play. Two challenges in the synthesis of Titanium reinforced BNNT composites are addressed: (i) The dispersion of high surface energy BNNTs to produce composites with homogenous distribution of BNNTs. (ii)The manufacturing of composites with significantly controlled interfacial reactions to aid in the strengthening of the Timatrix. Analysis and discussion based on the challenges addressed will be detailed based on reinforcement achieved and deformation/strengthening mechanisms involved.

\section{Addressing Challenges: Dispersion of high surface energy BNNTs}

A significant challenge in the integration of BNNTs as a reinforcement phase is their hydrophobic nature. The lack of a suitable dispersion route for the nanotubes without destructing its tubular morphology limits its applicability and processing. The ultra-fine diameters (5-10 nm) and extended lengths (up to $200 \mu \mathrm{m}$ ) of the nanotubes are responsible for the high surface energy leading to their easy aggregation via strong van der Waals forces. Hence, limiting their dispersion in most aqueous media. This study introduces two methods for the dispersion of BNNTs in aqueous media. Assessment of each method will be performed based on their suitability to the subsequent processing route (SPS) the composite powder will be subjected to.

\subsection{Chemical dispersion of BNNTs and synthesis of composite powder}

The dispersion of the nanotubes in aqueous media (DI water) was performed by the introduction of a non-ionic surfactant. The as-received BNNT fibrils consisted of highly 
agglomerated and entangled BNNTs (Figure 21). Introduction of the BNNTs to the aqueous media resulted in their segregation and non-wetting by the media, as shown in Figure 21.
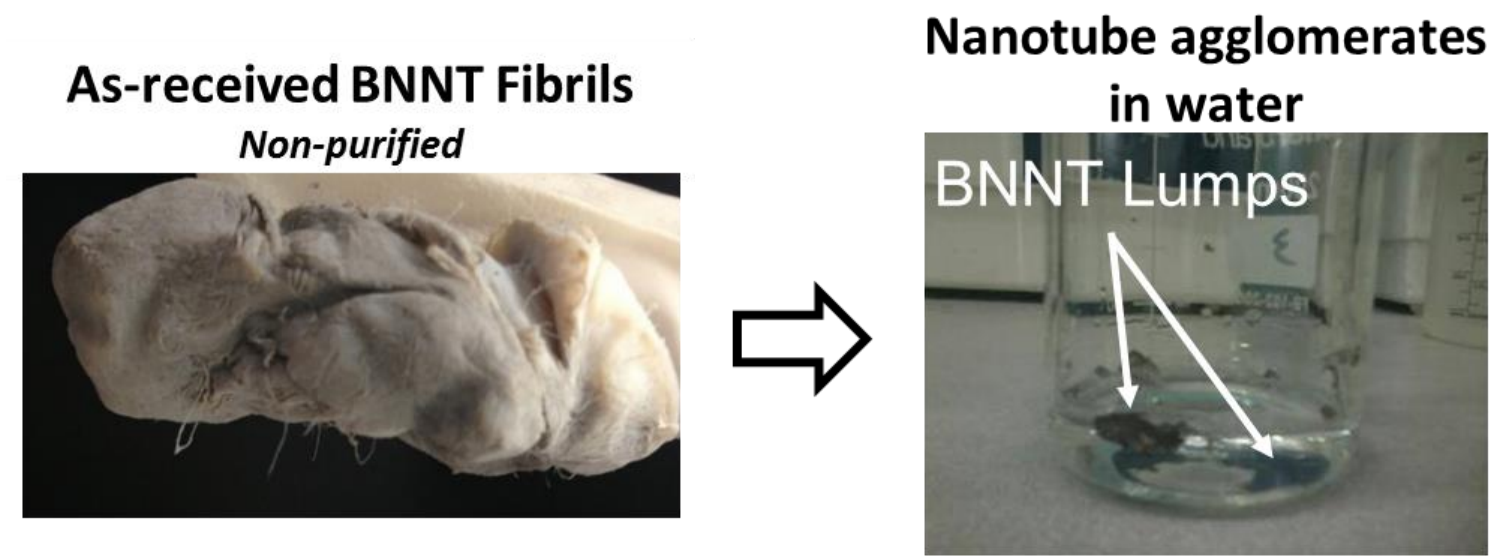

Figure 21 Pictorial representation of the as-received BNNT fibrils in the form of balls, the subsequent introduction to the aqueous medium resulted in their segregation and non-wetting as a result of the highly hydrophobic nature of BNNTs

Achieving a homogenous and uniform dispersion of BNNTs within the Ti matrix is of crucial importance to the resulting mechanical properties of the composite ${ }^{81}$. Also, to poor sintering, agglomerates within the structure are often found as nucleation sites for the failure of the composite ${ }^{81}$. Owing to the observed high hydrophobicity of BNNTs to DI water, a non-covalent dispersion route is used. As opposed to covalent dispersions, which include the introduction of chemical bonds between the nanotube wall and the functionalization agent, noncovalent agents introduce the adhesion of polymer-based molecules to the surface of the nanotubes ${ }^{82}$. The later method is preferred due to their ability to retain the crystallinity and integrity of the nanotube. 


\subsubsection{Role of surfactant in the dispersion of BNNTs}

The dispersion of BNNTs encompasses the achievement of three main aims: (i) Reduction of surface tension between the nanotube and aqueous media, promoting wetting of the nanotubes; (ii) disentanglement and destruction of agglomerates of long BNNTs by ultrasonic assisted route; and (iii) achieving a stable dispersion of BNNTs inhibiting re$\operatorname{agglomerates}^{76}$.

The hydrophobic nature of the nanotubes in aqueous media can be described by the equilibrium contact angle between the nanotubes and water ${ }^{55,83}$ :

$\cos \theta=\frac{\gamma_{s}-\gamma_{s} / l}{\gamma_{l}}$

For which, $\cos \theta$ described the angle between the surface of the solid being wetted and the liquid. This relationship between solid and liquid interfaces is a function of the surface energy of the solid $\left(\gamma_{s}\right)$, the surface tension of the liquid $\left(\gamma_{l}\right)$, and the solid-liquid surface tension $\left(\gamma_{s / l}\right)$. The interactions between the solid surfaces been wetted and the, liquid are characterized by their angle of wetting $(\theta)$. Higher wetting angles $\left(>90^{\circ}\right)$ are characteristic of poor wetting between the liquid and solid, while angles $\geq 90^{\circ}$ represent good wetting. Thus, it is intuitive to conclude from Eq. 9 that higher $\gamma_{s}$ values will result in higher wetting angles and; hence, result in preferred wetting of the surface. The surface tension of the solvent in use (DI water) has been reported as $72.7 \mathrm{mN} / \mathrm{m}^{83}$, whereas the surface energy of BNNTs is of $\sim 27 \mathrm{mN} / \mathrm{m}^{55}$. The significantly lower $\gamma_{s}$ of BNNTs are responsible for the hydrophobic nature observed in Figure 21. 
To address the poor wettability of the nanotubes in the media introduced, non-ionic surfactants are employed to reduce the surface tension between the nanotube and the solvent. A poly(ethylene oxide)(PEO), poly-propylene oxide (PPO) tri-block copolymer (PEO-PPO-PEO), commercially known as Pluronic is introduced as a non-ionic surfactant to the BNNT/DI water solution. Non-ionic surfactants are amphiphilic copolymers which contain both hydrophobic (apolar) and hydrophilic blocks (polar) ${ }^{84-86}$. Their high solubility in water solutions and their ability to adsorb to solid hydrophobic interfaces makes them suitable for the stabilization of colloidal solutions. The mechanism by which stabilization of the BNNT dispersion occurs is driven by micellization of the surfactant (Figure 22) ${ }^{76}$.

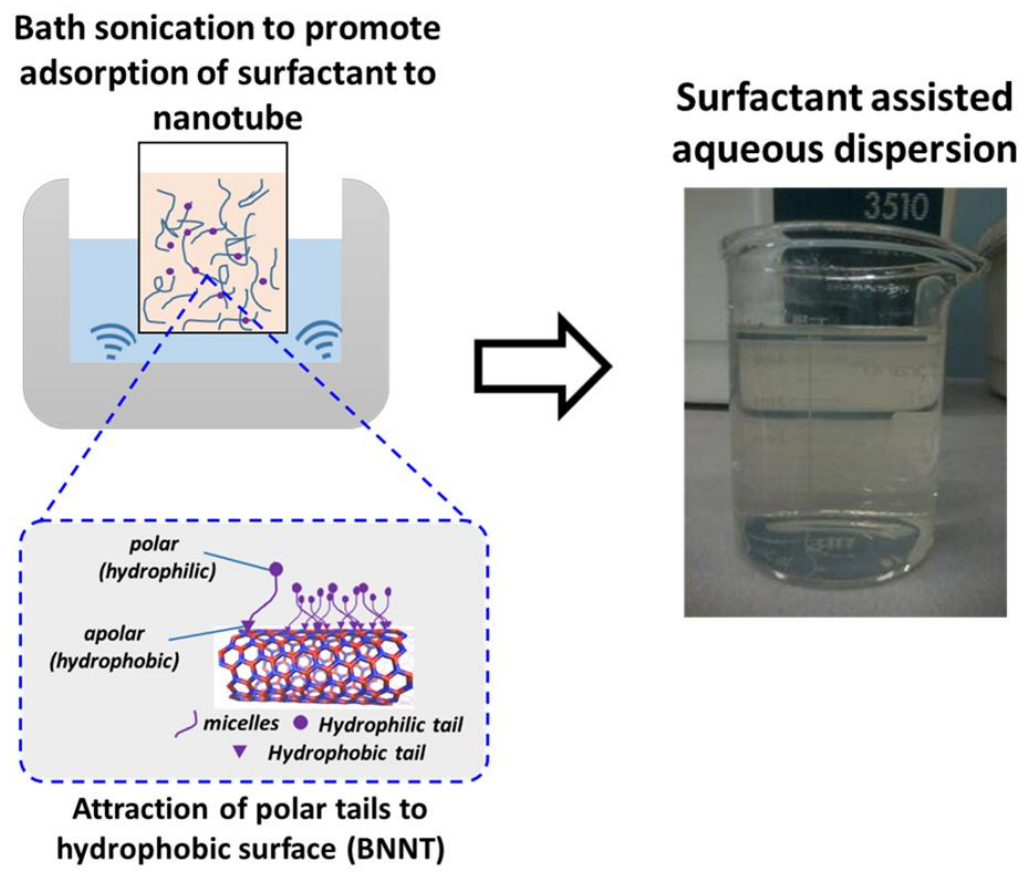

Figure 22 Schematic representation showing the mechanism by which a non-ionic surfactant introduced in the BNNT/DI water solution results in the reduction of surface tension between the nanotube and the aqueous media. 
Upon introduction of Pluronic particles into the aqueous solution, energy induced by bath ultrasonication promotes the solubility of pluronic in DI water. Initial reactions induce the formation of unimers (subunits of micelles) in the solution due to its low concentration. Exceeding a critical micellization composition (CMC) of Pluronic in the solution will induce the formation of micelles ${ }^{76}$. The CMC value is reported as $0.28-0.8$ wt. \% for Pluronic F127 used in this study ${ }^{85,86}$. The final concentration of the solution in this study demonstrates this value is exceeded in experimentation ( $\sim 3 \mathrm{wt} . \%)$ and therefore, the formation of micelles is assumed to be attained ${ }^{84}$. PPO groups present in the copolymer are responsible for the hydrophobic chains in the micelles, while PEO groups render the hydrophilic chains ${ }^{85}$. The hydrophobic chains (PPO) are assumed to interact with the hydrophobic surface of the nanotubes using mutual $\pi-\pi$ interactions ${ }^{84-86}$. The adherence of the hydrophobic micelle molecules to the surface of the nanotubes exposes the hydrophilic tails (PEO chains) to the solvent. Thus, reducing the surface tension of the nanotubes and promoting their stability by steric forces ${ }^{76}$. Lin et al ${ }^{84}$ has demonstrated the adsorption of Pluronic micelles to the surface of hydrophobic carbon black nanoparticles to increase surface thicknesses to up to $\sim 26 \mathrm{~nm}$ in a $1 \mathrm{wt} . \%$ pluronic addition. Hence, the stabilization process is understood as the wrapping of polymer chains onto the surface of the hydrophobic solids and has a two-fold advantage: (i) reducing interfacial surface tension between the nanotube and the aqueous media, and (ii) induction of high repulsive forces among dispersed BNNTs preventing their re-agglomeration. ${ }^{84}$ As observed in Figure 22, the clear solution obtained signaled the dispersion of BNNTs without significant agglomerates (visible at the naked eye). 


\subsubsection{Homogeneity and dispersion of BNNTs in composite powder}

The dispersion of BNNTs in aqueous media was soon followed by the addition of Ti6Al4V spherical powder. The adherence of dispersed BNNTs on to the surface of Ti particles was aided by a magnetic stirring process for at least $15 \mathrm{~min}$. The aqueous solution was then oven dried, and 2-hour heating to $200^{\circ} \mathrm{C}$ was used to remove the organics from the surfactant.

Removal of residual surfactant from the composite powder is of crucial importance owing to the elevated temperatures involved in the sintering of composites. The presence of residual surfactants could lead to increased defects within the structure. Thus, postpurification processes are often introduced, such as vacuum filtration and acid washing. While vacuum filtration coupled with repeated washing routines have shown potential in the removal of residual surfactant in a colloidal solution, this process entails the reagglomeration of nanoparticles. Also, the adsorption of surfactant derived micelles on the surface of the BNNTs make impossible their complete mechanical removal. On the other hand, the introduction of acids as purifying agents has often resulted in the introduction of surface defects on the nanoparticles. Thus, showing undesirability for their use.

Thermal annealing of the composite powder was introduced in this study as an alternative route to remove the organic surfactant from the powder. Given that the melting point of the non-ionic surfactant is of $\sim 55^{\circ} \mathrm{C}$ and its boiling point $>149^{\circ} \mathrm{C}$, an annealing temperature of $200^{\circ} \mathrm{C}$ and a 2-hour heating period were chosen for the evaporation of organics and removal of surfactant from the composite powder. Figure 23a shows the resultant composite powder after the annealing process was completed. A large number of 
localized agglomerates are encountered throughout the powder mixture. Closer observation of the powder particles (Figure 23b) reveals the presence of agglomerated nanotubes in companion to residual surfactant particles.
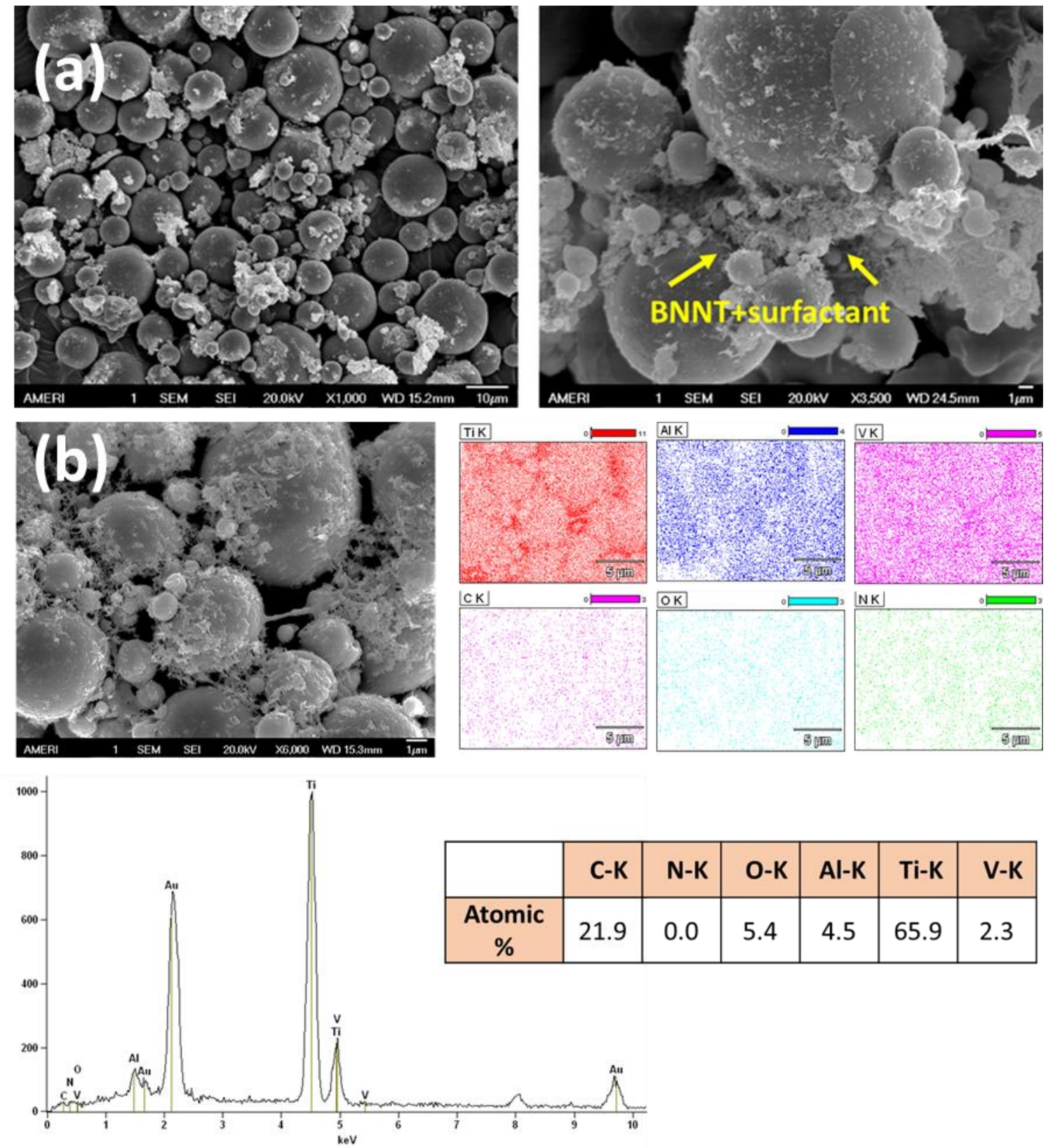

Figure 23 (a) Resultant Ti-BNNT composite powder after drying and thermal process was completed showing residual surfactant. (b) EDS mapping of the composite powder revealed the presence of Carbon as a result of residual surfactant 
Although an exhaustive thermal annealing step was introduced in the processing of the composite powder, the surfactant was still present as aggregates throughout the surface of the Ti powder encapsulating large BNNT agglomerates. The large presence of surfactant clusters precipitated on the composite powder was evidenced by the carbon content $(\sim 22$ at. \%) in EDS analysis. This anomaly can be attributed to the surfactant's behavior in the presence of heat. The micellization process of Pluronic has been reported to exhibit dependence on the temperature of the solution at which it is exposed ${ }^{87}$. Low surfactant concentrations $(<50 \mathrm{mg} / \mathrm{mL}$ ) experienced increasing growth of micelles into spherical/ellipsoidal particles above $\sim 52^{\circ} \mathrm{C}^{87}$. Jebari et al. ${ }^{87}$ reached to the conclusion that although temperatures above $>45^{\circ} \mathrm{C}$ allowed a larger degree of micellization of the surfactant. The process could also entail to a larger number of PPO hydrophobic chains associating with other micelles, thus, generating clusters and swelling of micelles.

In this study, the high energy introduced during the dispersion of the nanotubes via bath sonication can be considered as the source of heat in the solution. The dissipation of ultrasonic waves, often at frequencies $>20 \mathrm{kHz}$, is known to induce the formation of cavities in an aqueous solution ${ }^{88}$. After experiencing continuous expansion and retraction, cavities in the solution experience collapsing. Such a process has been largely reported to result in localized heating to temperatures up to $5000 \mathrm{~K}$ and pressures of $\sim 2 \mathrm{~atm}^{88}$. Although these extremely high temperatures are experienced at the local centers of cavities, their contribution to the solution can extend to around $200 \mathrm{~nm}$ from the surface of the cavity with temperatures of up to $2000 \mathrm{~K}^{88}$. This process has been reported to have durations of $<2 \mu \mathrm{s}$, and hence reduce the direct impact of high temperatures on the solution ${ }^{88}$. Hence, 
heat derived from the bath sonication process can be deduced as responsible for the micelle growth and agglomerated clusters found in the final composite powder.

On the other hand, evidence of individual nanotubes wrapped on the surface of Ti particles was also observed (Figure 24). It is understood from the micellization mechanism described above, that the critical micelle composition was exceeded and allowed the dispersion of nanotubes to take place. Increased thickness of nanotubes as observed in Figure $24 \mathrm{a}$ and $\mathrm{b}$ serve as evidence of the wrapping of nanotubes by the copolymer molecules introduced.
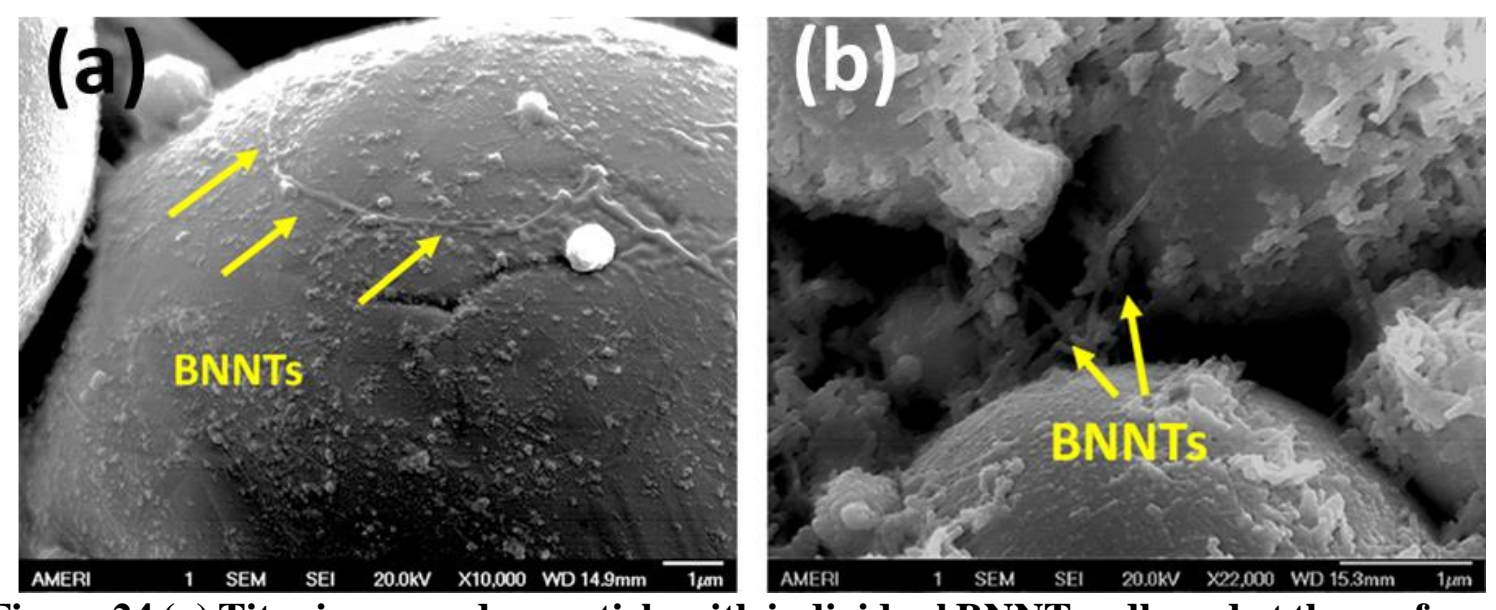

Figure 24 (a) Titanium powder particle with individual BNNTs adhered at the surface of the powdered particle. (b) Evidence of BNNTs interfacing with the powder particles and surfactant

Survival of BNNTs after been subjected to long periods of high energy sonication can be attested in the synthesis of the composite Ti-BNNT powder. The mechanical stability of the nanotubular structure is still of interest and could represent a reinforcement to the matrix. Although complete dispersion of nanotubes was hindered by micellar aggregates in the presence of localized heat, the subsequent sintering of the composite powder was performed, and the suitability of the surfactant-assisted dispersion is discussed. 


\subsubsection{Effect of surfactant during consolidation}

Assessment of the fracture surface of sintered Ti-BNNT composites obtained via surfactant assisted route is shown in Figure 25. Sintering of the composite powder was performed at maximum temperatures of $950^{\circ} \mathrm{C}$ to evaluate the suitability of the dispersion route to high-temperature processing. The sintered composite resulted in specimens with a high relative density of $\sim 98 \%$. Although high relative densities were achieved, fracture surface evaluation demonstrated the presence of large BNNT agglomerates within the matrix. The presence of agglomerates in the composite is attributed to the poor dispersion achieved in the introduction of surfactants. High energy-induced heating of the solution leading to the swelling and aggregation of surfactant micelles were deduced as the primary source of poor BNNT dispersions. Also, agglomerates in the composite are found to act preferential sites for the presence of cracks. In the presence of agglomerates, nanotubes found away from the Ti/BNNT interfaces render lower interactions between the nanotubes and the Ti matrix. Thus, inhibiting the bearing of stresses and promoting more significant deformations as a result of non-covalent shearing between nanotubes.

Moreover, elemental analysis of the fracture surface via EDS demonstrated agglomerate sites as carbon-rich regions. In which, residual free-surfactant and that strongly wrapped within the nanotubes experience calcination during sintering of the composite. 

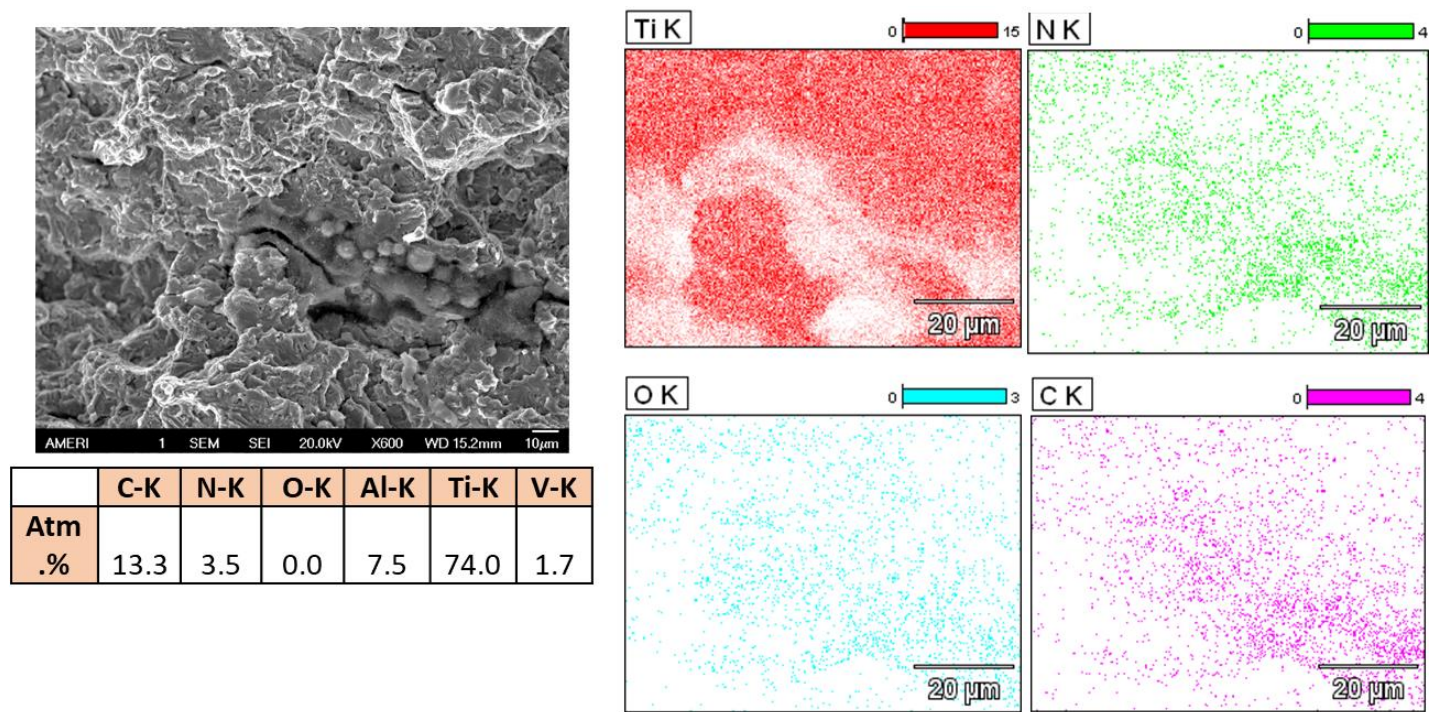

Figure 25 EDS mapping of the as-sintered fracture surface of the Ti-BNNT composite synthesized via surfactant assisted route. Carbon as an impurity is encountered

The calcination process can be described by the nature of the surfactant's thermal decomposition. Thermogravimetric analysis (TA) of the Pluronic surfactant was performed by exposing the initial powder to temperatures up to $600^{\circ} \mathrm{C}$ (Figure 26). Evaluation of the curve resolves major losses in weight to occur between $250-400^{\circ} \mathrm{C}$. For which, the decomposition of organics and polymeric chains takes place, resulting in residual carbon of $\sim 1.5 \%$ the initial weight. The presence of carbon as a contaminant in the composite is considered detrimental due to the thermodynamic feasibility to form $\mathrm{TiC}$ reaction products within the composite. The poor solubility of carbon within Ti at elevated temperatures (> $500^{\circ} \mathrm{C}$ ) is known to result in the precipitation of $\mathrm{TiC}$ phases in its interaction with $\alpha$ and $\beta$ phases of the matrix. 


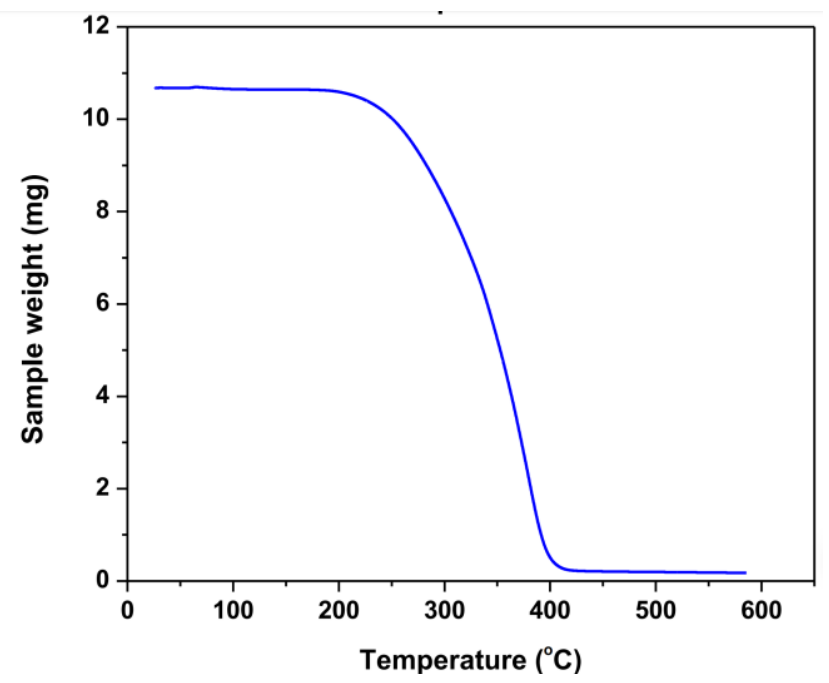

Figure 26 TGA curve of the Pluronic F127 used in the dispersion of BNNTs, curve demonstrates major thermal degradation to occur between $200-400^{\circ} \mathrm{C}$ with residual Carbon on $1.5 \%$ of its initial weight.

Although the carbon concentration is considerably low $(\sim 1.5 \%)$, the major losses in weight of the surfactant upon exposure to elevated temperatures result in the origination of defects in the microstructure. Figure 27 shows the microstructure of the Ti-BNNT composite after sintering was completed. The microstructure reveals large voids throughout the microstructure. Defect sites (i.e., voids) are a result of the evaporation of volatile organic components within the surfactant during sintering. Spark plasma sintering, as a consolidation route, entails a constrained sintering process limiting the escape of gases. Hence, resulting in the presence of large voids throughout the microstructure. 

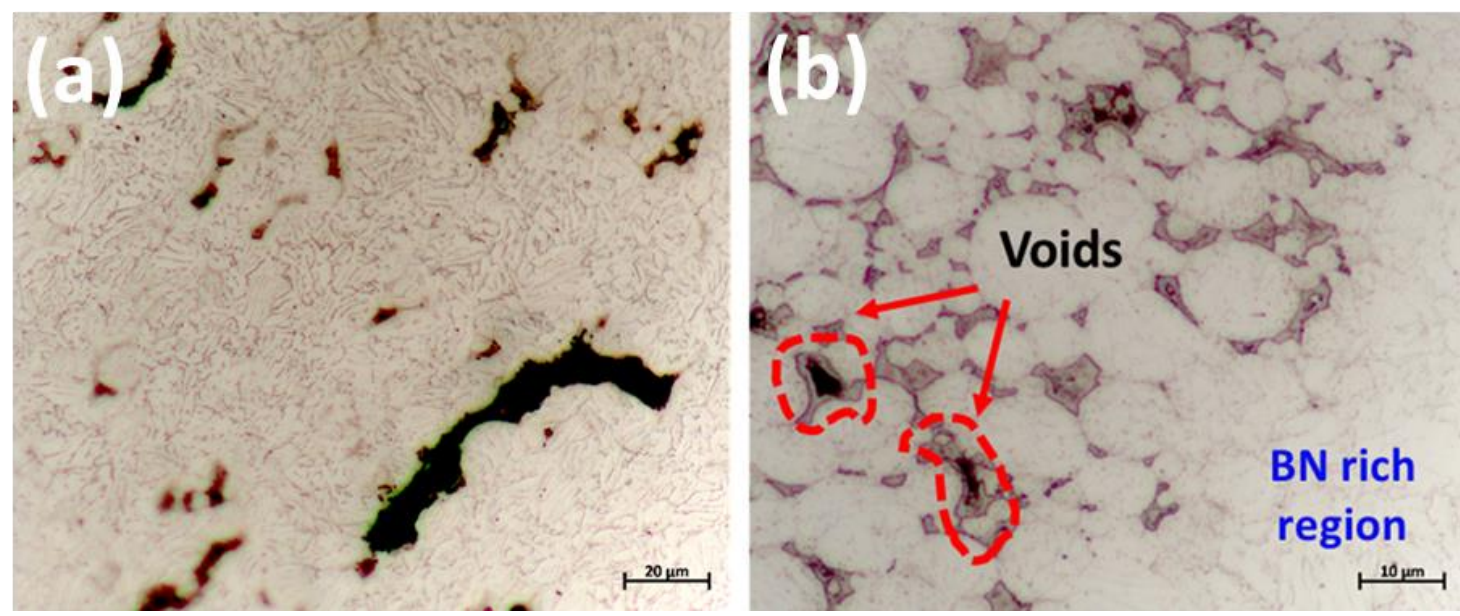

Figure 27 Optical micrographs of polished and etched surfaces of the as-sintered TiBNNT composites synthesized via surfactant-assisted route showing the significant presence of voids as a result of volatile organics in residual surfactant.

It is therefore concluded that the dispersion via surfactant addition is not suitable for the manufacturing of Ti-BNNT composites. Specifically, manufacturing of composites via powder metallurgy route is deemed to not benefit from the surfactant-assisted dispersion, unless a more appropriate surfactant removal process is introduced. In light of the poor dispersion and contaminants (residual Carbon) encountered in the synthesis of the composite, a dispersion process averting the use of surfactants as dispersion aids will be further pursued.

\subsection{High energy induced dispersion of BNNTs by tip sonication}

An alternative route is introduced for the dispersion of BNNTs in an organic solvent. Acetone is introduced as a solvent due to its high affinity to the nanotubes and ability to generate a protective layer for oxidation in the Ti particles. Owing to the high energy transfer during the ultrasonic wave propagation, ultrasonication by tip sonication has been the desired technique in the dispersion of high surface energy nanoparticles. 
The advantage of introducing a sonicating probe into the solution includes the ability to reduce dispersion times by $\sim 128 \times$ of the required time in a surfactant/bath sonication dispersion. Also, the reduced damage to the nanotubes was reported by Caneba et al. ${ }^{72}$ in the dispersion of CNTs. Figure 28 shows the steps carried out in the dispersion of BNNTs by ultrasonication vibrations. First, entangled BNNTs are observed in the form of fibrils. Introducing a sonication probe for $45 \mathrm{~min}$ with frequencies of $19 \mathrm{kHz}$ and amplitudes of $\sim 47.2 \mu \mathrm{m}$ resulted in the dissociation of entangled BNNTs and allowed their stable dispersion (shown by cloudy solution). Ti6Al4V powder particles were later introduced into the stable BNNT/acetone solution, and magnetic stirring was performed. The complete adherence of dispersed BNNTs onto Ti particles was achieved after 1 hour and is evidenced by the clear supernatant in Figure 28. After evaporation of volatile acetone via oven drying, evaluation of the composite powder took place. The distribution of BNNTs was found to be homogeneous throughout the Ti powder. In which, individual nanotubes are observed on the surface of the powder particles, as well as small bundles. 


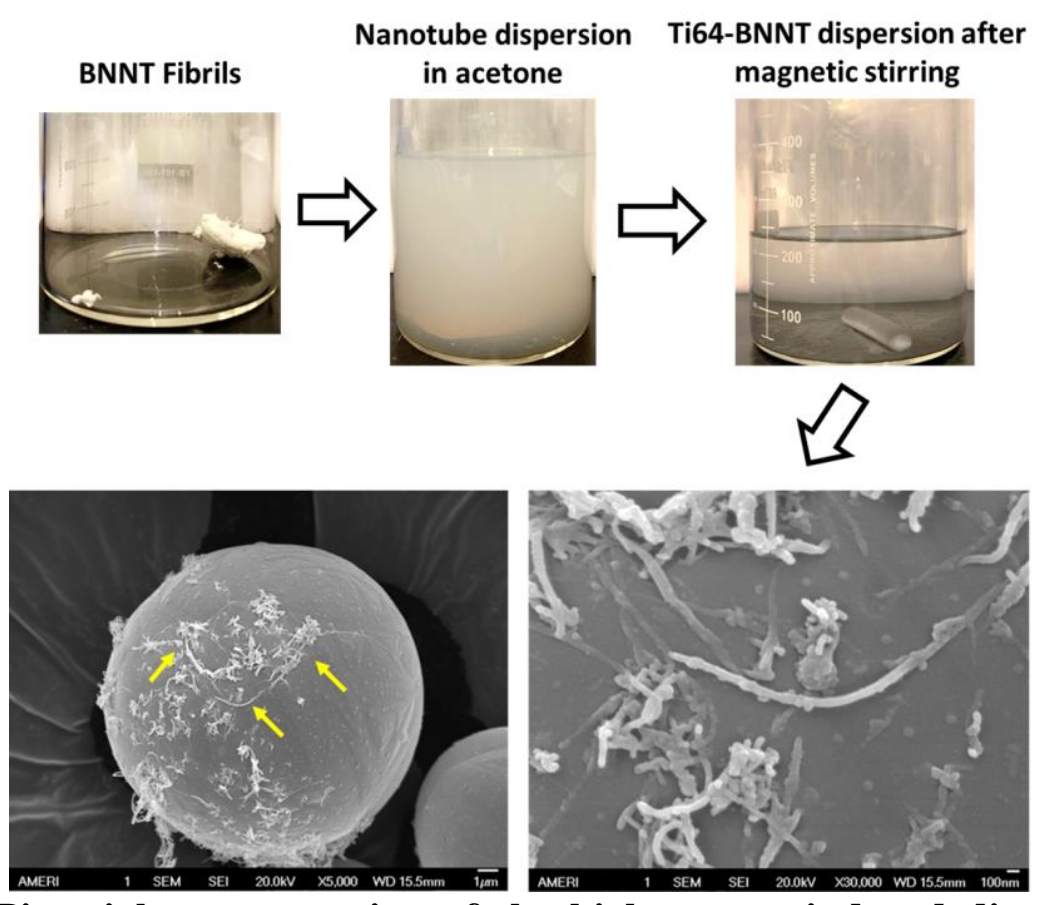

Figure 28 Pictorial representation of the high energy induced dispersion of high surface energy BNNTs in acetone. The resultant composite powder shows the integrity of the nanotubes to high energy sonication and the achieved dispersion.

\subsubsection{Role of ultrasonic waves and cavitation in breaking agglomerates and dispersion of BNNTs}

The enhanced dispersion of high surface energy BNNTs in the composite powder can be attributed to the mechanical and chemical disturbances introduced during the ultrasonication process. The propagation of ultrasound through a liquid is known to result in the induction of positive and negative pressures within the liquid ${ }^{72,73}$. Physical break down of the liquid is then the nuclei of voids when large negative pressures are experienced. The process by which the propagation of waves generates voids within a liquid is known as acoustic cavitation ${ }^{72,73,88}$. The importance of the cavities for the dispersion of nanoparticles and specifically BNNTs lies in their ability to be a carrier of high energies and gases derived from the solvent used that will interact with the dispersing particles. At frequencies $>19 \mathrm{kHz}$, acoustic cavitation experiences growth and sudden 
collapsing after reaching critical sizes of $\sim 170 \mu \mathrm{m}^{72,73}$. The collapsing of high energy carrier cavities is known to result in the localized heating (up to $5000 \mathrm{~K}$ ) present for short periods of $\sim 2 \mu \mathrm{s}^{72,73,88}$. In the presence of solid-liquid solutions, acoustic cavities are capable of imparting high accelerations to the solid nanoparticles capable of imparting enough energies to dissociate nanoparticles.

The recurrence of a large number of cavities is highly dependent in the intensity of the acoustic vibrations introduced ${ }^{72,73,88,89}$ :

$I=\frac{1}{2} \rho c(2 \pi f A)^{2}$

The mathematical relation between the density of the liquid medium ( $\rho$ ), the speed of sound within the liquid (c), the frequency $(f)$ and the amplitude of vibrations, will ultimately dictate the degree of collapsing cavities in the solution. In this study, acetone is characterized by having a density of $0.78 \mathrm{~g} / \mathrm{cm}^{3}$ and dissipates sound in speeds of $\sim 1170$ $\mathrm{m} / \mathrm{s}^{89}$. Thus, the intensity of acoustic vibrations in the acetone/BNNT solution is computed as $1457.13 \mathrm{~W} / \mathrm{cm}^{2}$. The high intensity of cavitation introduced in the solution is then responsible for the acceleration of entangled nanotubes driving them with speeds of up to $400 \mathrm{~km} / \mathrm{h}^{89}$. The high acceleration induced to the nanotubes is proposed as the main driving mechanism for the mechanical dissociation of entangled BNNTs. In addition to providing enough energy for the mechanical propulsion of nanotubes, the high energy carried by the cavities are known to induce chemical effects on the solvent ${ }^{72,73,88,89}$.

In the presence of high energies, the localized heating and pressure induced by exploding cavities is known to result in the formation highly reactive species. Thus, 
evaluating the potential species present during the sonication of the acetone/BNNT would allow us to understand the mechanisms by which the highly hydrophobic BNNTs are wetted and dispersed. The introduction of high frequency $(>19 \mathrm{kHz})$ acoustic cavitation in acetone $\left(\mathrm{C}_{3} \mathrm{H}_{6} \mathrm{O}\right)$ has been reported to result in the formation of Hydrogen atoms, Hydroxyl radicals, and even carbon-centered radicals ${ }^{72,73,88,89}$. Free radicals are highly reactive species that are present in higher concentration nearing the surface of cavities. However, the escape of free radicals to the bulk solution is known to result in reactions between the compounds within the solvent (i.e., BNNTs). Free radicals are highly reactive species introduced during cavitation that are bound to interact with defects on the surface of BNNTs.

The dissociated hydroxyl radicals (-OH) from acetone as a result of the high energy imploded cavities can be responsible for the improved wetting of the BNNTs in aqueous solutions. The in-situ functionalization of hydrophobic BNNTs by -OH radicals result from the ability of free radicals present to interact covalently with electron deficient sites (i.e., Boron) in the BN structure (defect sites) ${ }^{90,91}$. Plasma treatments and hydrothermal reactions have been shown as alternative routes for the functionalization of nanotubes via formation of $-\mathrm{OH}$ radicals ${ }^{91,92}$. Hydroxylation is further promoted by the open atmosphere in which the dispersion takes place, allowing for $\mathrm{O}_{2}$ species to interact with the solution. Similar to the hydroxylated process observed in the sonication assisted dispersion of BNNTs, the sonication of h-BN sheets in water has resulted in their hydrolysis ${ }^{92}$. Lin et $\mathrm{al}^{93}$. demonstrated that $\mathrm{BN}$ bonds nearing defect sites were prone to the attack of $\mathrm{O}_{2}$ atoms derived from water molecules resulting in their dissociation and fragmentations. A similar mechanism by which hydroxyl groups derived from free radicals impart hydrophilic nature 
in initially hydrophobic BNNTs ${ }^{91}$. The energetically favorable interaction between the hydroxyl radicals and the nanotube surface has been reported to give binding energies of $0.93 \mathrm{eV}$ between acetone derived molecules and B sites in interaction ${ }^{79}$.

The significance of surfactant-free dispersion of BNNTs with high surface energy and highly hydrophobic nature lies in the scalability of the technique and the ease of volatilization of the solvent from the composite powder. Thus, mitigating the contaminants and defects encountered in the surfactant-assisted dispersion of BNNTs. Following the successful dispersion of BNNTs on the $\mathrm{Ti}$ powder, challenges concerning the thermodynamics of reactions are addressed for the sintering of the composite.

\section{Addressing Challenges: Controlling thermodynamically feasible reactions}

The high reactivity of Titanium during thermal processing in the presence of nanoparticles has posed a challenge in the manufacturing of composites. The chemical inertness and thermal resistance to up to $900^{\circ} \mathrm{C}$ of BNNTs, suggests the potential of these nanostructures to serve as reinforcing nanoparticles of metals. In this study, the lowtemperature sintering $\left(<900^{\circ} \mathrm{C}\right)$ of Titanium alloys is introduced as an alternative route to high-temperature processing $\left(>900^{\circ} \mathrm{C}\right)$ commonly used in the manufacturing of bulk $\mathrm{Ti}$ pieces.

\subsection{Low-Temperature sintering of Ti64Al4V}

Processing of Ti6Al4V via the pressureless, hot press, hot isostatic pressing, and pressure assisted sintering (SPS) has been widely investigated. Figure 29 presents a visual summary of the sintering techniques currently used for the sintering of Ti6Al4V prealloyed powders ${ }^{94-99}$. The graph differentiates and clearly states the high temperatures 
required in pressureless sintering processes to achieve scattered degrees of densification $(62-99 \%)$. The scattered data points in pressureless sintering, are a result of the various sintering conditions often used, where extremely long sintering periods (up to $90 \mathrm{~min}$ ) and temperatures of $\sim 1371^{\circ} \mathrm{C}$ are required to achieve nearly fully dense structures. While lower temperatures $\left(1250^{\circ} \mathrm{C}\right)$ for periods of 2 hours results in merely $62 \%$ relative density ${ }^{100,101}$.

Some efforts to reduce the sintering periods have introduced hot-pressing, and HIP techniques with potential to achieve full densification of the alloy by the introduction of additional compacting pressure processing steps. In which, isostatic pressures of $\sim 800$ $\mathrm{MPa}$ are introduced by the continuous supply of inert gas and heating of the Ti powder containing chamber ${ }^{95,99}$. Although this process has shown potential in the sintering of fully dense Ti structures (up to $99 \%$ densification), the technique still extends the processing periods to 3 hours. Moreover, the simultaneous pressure and heating during the sintering of metallic powders have been investigated via Spark Plasma Sintering routes. In which, near net shape structures are manufactured in periods between $10-30 \min ^{44,52,53,94,96,98}$. This signifies a reduction in sintering times by up to $4 \times$ that required in other sintering routes.

Outlining the several processes used in literature for the sintering of Ti6Al4V as a function of temperature clearly shows two temperature-dependent regimes:

- Sintering rule of thumb $\left(>1000^{\circ} \mathrm{C}\right)$; chosen temperatures follow sintering's rule of thumb where sintering temperatures are $70 \%$ of the melting temperature $\left(\mathrm{T}_{\mathrm{m}}\right)$ of the metal. 
- Pressure-assisted sintering $\left(800-100^{\circ} \mathrm{C}\right)$; mild sintering temperatures are chosen as the reduction in porosity is achieved by pressure assisted methods.

Close observation of the comparative graph (Figure 29) distinguishes a third regime denominated as "processing gap" at temperatures $<800^{\circ} \mathrm{C}$. In which, sintering of prealloyed Ti6Al4V has not been explored. Keeping in mind that the ultimate goal of this study is to achieve the manufacturing of reinforced Titanium-BNNT composites with control interfacial reactions. This study introduces the concept of low-temperature sintering: where sintering temperatures below $800^{\circ} \mathrm{C}$ are chosen in combination with elevated sintering pressures $(300-555 \mathrm{MPa})$ to achieve full densification of Ti6Al4V and ultimately the sintering of Ti-BNNT. Sintering of the BNNT reinforced Ti composite at common SPS conditions of $\sim 950^{\circ} \mathrm{C}$ exposes the nanotubes to temperatures exceeding the experimentally observed oxidation, as well as presents thermodynamically feasible conditions for the formation of reaction products within the interfaces. Hence, exploring lower temperature regimes, well below the oxidation of BNNTs, could result in composites with minimal reaction products as desired. 


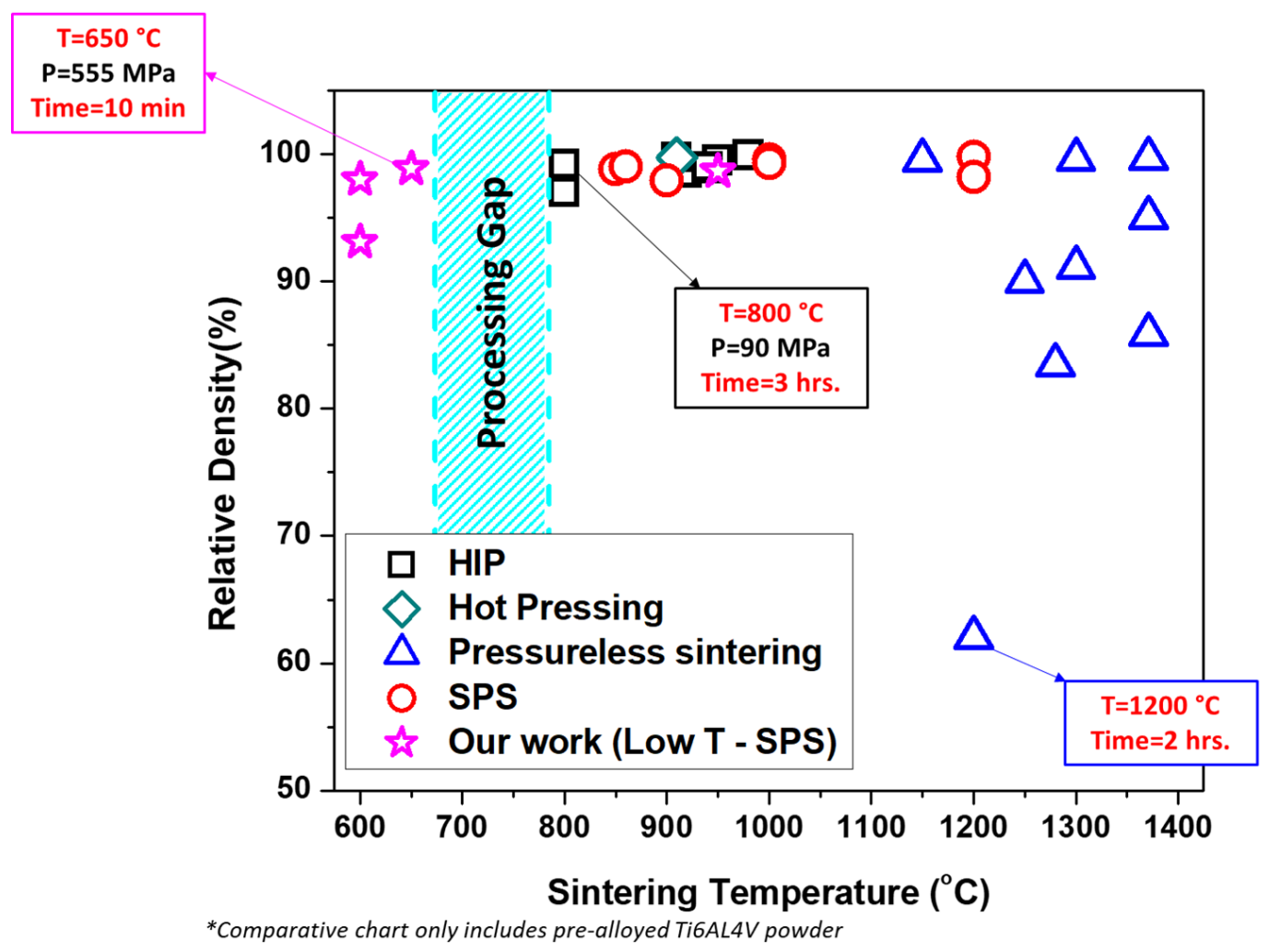

Figure 29 Comparative chart of pressureless and pressure assisted sintering routes used in the past for the densification of Ti6Al4V. A processing gap is identified and investigated with potential advantages for Ti-BNNT composites

\subsubsection{Densification and microstructural evolution}

Owing to the promising advantages of low-temperature sintering of $\mathrm{Ti}$, sintering of Ti6Al4V was carried out at temperatures between $600-650^{\circ} \mathrm{C}$ and pressures of up to $555 \mathrm{MPa}$. Table 5 describes the resulting properties of the T6Al4V samples sintered at different temperatures and pressures. 
Table 5 Summary of microstructural characterization of low temperature sintered Ti6Al4V

\begin{tabular}{ccccc}
\hline \hline $\begin{array}{c}\text { Temperature } \\
\left({ }^{\circ} \mathbf{C}\right)\end{array}$ & $\begin{array}{c}\text { Pressure } \\
(\mathbf{M P a})\end{array}$ & $\begin{array}{c}\text { Relative Density } \\
(\boldsymbol{\%})\end{array}$ & $\begin{array}{c}\text { Grain size } \\
(\boldsymbol{\mu m})\end{array}$ & $\begin{array}{c}\text { Hardness } \\
(\mathbf{G P a})\end{array}$ \\
\hline 600 & 300 & 93.1 & $9.6 \pm 3.1$ & $2.7 \pm 0.11$ \\
\hline 600 & 500 & 98 & $10.6 \pm 3.7$ & $3.7 \pm 0.02$ \\
\hline 650 & 555 & 98.9 & $11.73 \pm 2.7$ & $3.7 \pm 0.04$ \\
\hline 950 & 60 & 98.65 & $\begin{array}{c}\text { Long.: } 7.4 \pm 2.6 \\
\text { Trans.: } 2.3 \pm 0.9\end{array}$ & $3.2 \pm 0.09$ \\
\hline \hline
\end{tabular}

It is important to highlight the high level of densification ( 98\%) achieved by merely $600^{\circ} \mathrm{C}$ and exerting $500 \mathrm{MPa}$. In which, relative densification values of specimens sintered at 300 vs. $500 \mathrm{MPa}$ suggest a dependence on the deformation required for powder particles to consolidate. A subsequent increase in sintering temperature by $50^{\circ} \mathrm{C}\left(650^{\circ} \mathrm{C}\right)$ and small increases in pressure by $5 \mathrm{MPa}(555 \mathrm{MPa})$ resulted in a further increase in the relative density by at least $1 \%$ to almost $99 \%$ relative density.

To understand the mechanisms by which densification of the Ti6Al4V occurs at low temperatures and high pressures, densification curves were studied as a function of temperature (Figure 30). 


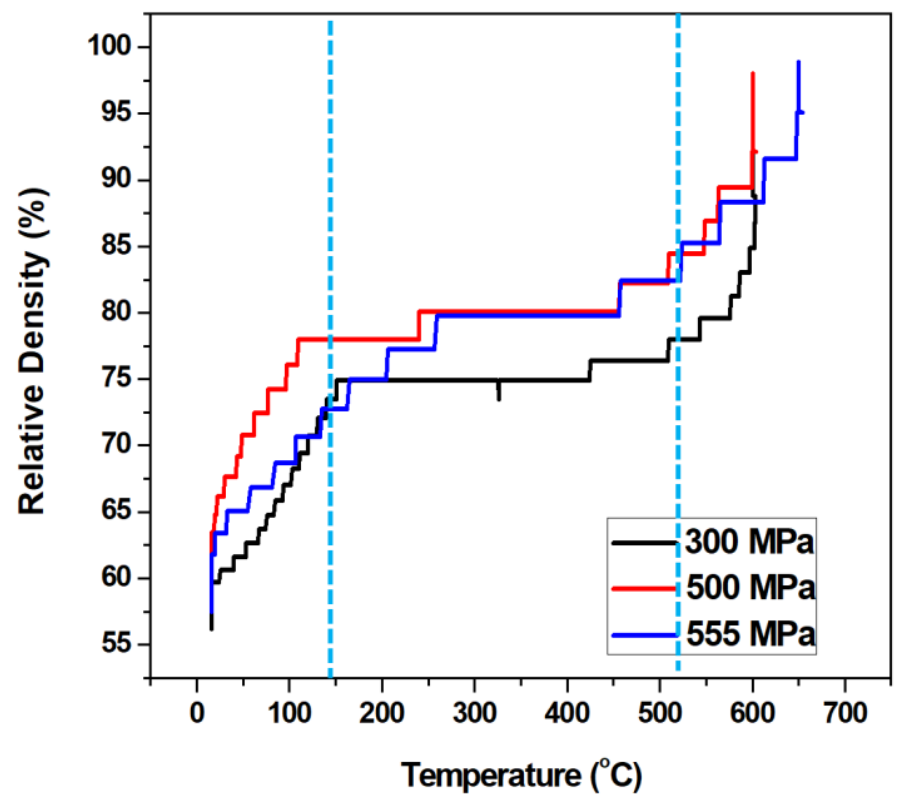

Figure 30 Densification curves of low temperature sintered Ti6Al4V showing three distinct regimes of densification

Densification curves are of prime importance as they can provide real-time densification phenomena occurring to the specimen during sintering. Three densification regimes are identified in Figure 30 to play a role in the densification of the alloy. The first stage, between temperatures of $\mathrm{RT}-150^{\circ} \mathrm{C}$, where the packing and rearrangement of the powder particles take place ${ }^{102}$. At the same time pressure starts increasing, allowing packing densities to reach up to $75-80 \%$ densification. Increasing packing density before any sintering occurs in the powder decreases the inter-particle distance allowing for diffusion to occur at a much faster rate.

Soon after, the second sintering regime occurs between $150^{\circ} \mathrm{C}-500^{\circ} \mathrm{C}$. For which the rate of densification is the lowest $(\sim 0.3 \% / \mathrm{min})$. During this regime, necking and plastic deformation of particles is presumed to occur with minimal impact in the densification of the sample given that stresses are low. Figure 31 demonstrates the fracture surface of a Ti6Al4V specimen sintered at $600^{\circ} \mathrm{C}$ and $300 \mathrm{MPa}$. In which, the poor relative density 
achieved can be attributed to the poor sintering obtained at the selected sintering conditions. Sintered specimens demonstrate outcomes described in the second sintering regime, where initial necking and deformation of particles can be observed.

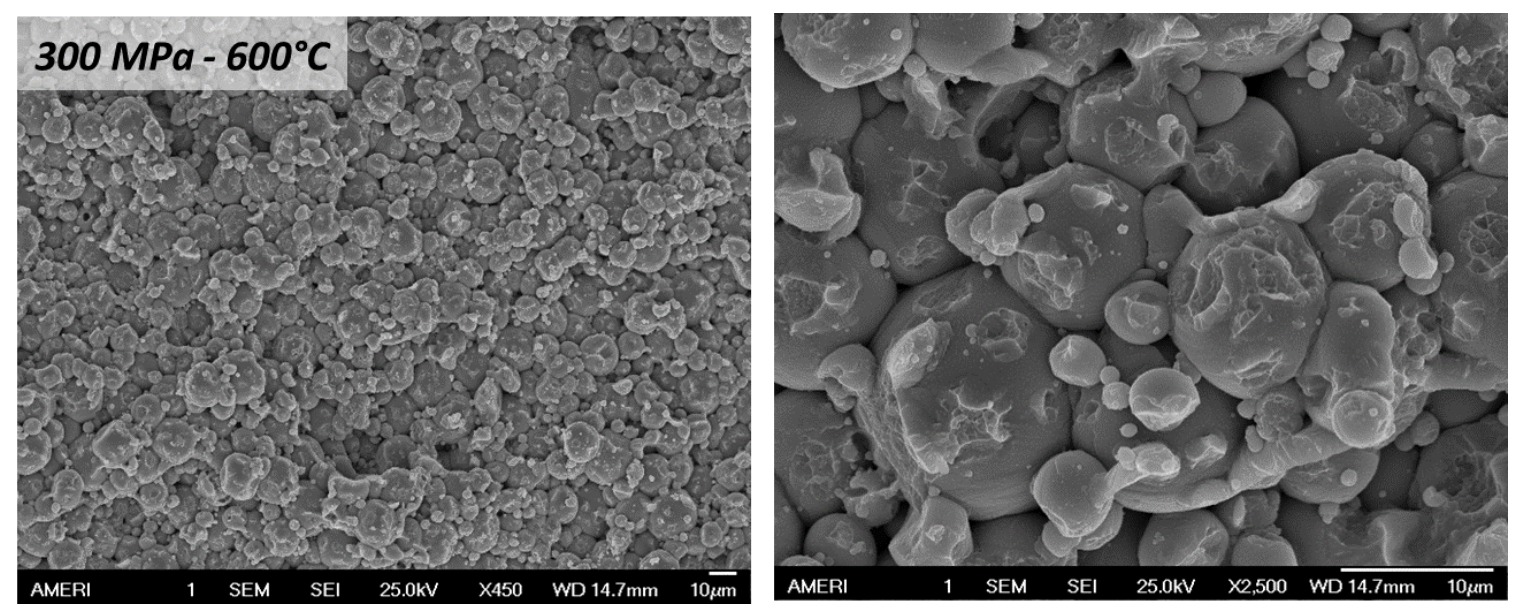

Figure 31 Fracture surface of the Ti6Al4V sample sintered at $600^{\circ} \mathrm{C}$ and $300 \mathrm{MPa}$, showing poor interparticle interactions with signs of surface diffusion (necking).

The notion that sintering of materials occurs under the simultaneous effect of heat and plastic deformation provides the guideline to the understanding of sintering mechanisms undergone in this study. For successful densification of Ti6Al4V to occur at low temperatures, the effective stress exerted during SPS must exceed the yield strength of the $\mathrm{Ti}^{102}$. That is, pressure assisted densification can be achieved if the sintering pressure is enough to induce yielding of the powder particles. The temperature dependence of the yielding strength of Ti6Al4V has been well reported over a wide range of temperatures. Literature has reported this value for Ti6Al4V to be between $550-680 \mathrm{MPa}$ at temperatures of $600^{\circ} \mathrm{C}^{102}$. Thus, the low pressures used are not enough to induce particle yielding and densification in the specimen. This intermediate mechanism is denominated as surface diffusion, where pressures are not significant enough to induce major plastic flow required ${ }^{102}$. 
Increasing temperatures above $>500^{\circ} \mathrm{C}$ gives rise to the third regime. In which, the hold periods at constant max. Temperature and pressure induce the self-diffusion of $\mathrm{Ti}$ particles and allowed densification to occur. This third regime is observed to occur at rates of about $2 \% / \mathrm{min}$ and is achieved by specimens sintered at $600^{\circ} \mathrm{C}-500 \mathrm{MPa}$ and $650^{\circ} \mathrm{C}-$ $555 \mathrm{MPa}$. Figure 32 shows the fracture surface of both specimens sintered at $600^{\circ} \mathrm{C}-500$ $\mathrm{MPa}$ and $650^{\circ} \mathrm{C}-555 \mathrm{MPa}$. Features such as dimples within the fracture surface are characteristic of a ductile fracture and serve as evidence of near full densification achieved. The increase in pressure to 500 and $555 \mathrm{MPa}$ near the reported yielding of Ti is responsible for allowing mass transport and moderate diffusion as densification mechanisms to occur $^{102}$. The significance of this finding lies the ability to reduce sintering temperatures by $\sim 40 \%$ that of conventional SPS conditions and $\sim 50 \%$ of non-pressure assisted sintering techniques. Moreover, it is important to highlight that the full densification of the Ti6Al4V was achieved in merely 10 min of sintering. Hence, reducing sintering times and temperatures for what was considered a thermally exhaustive routine. 


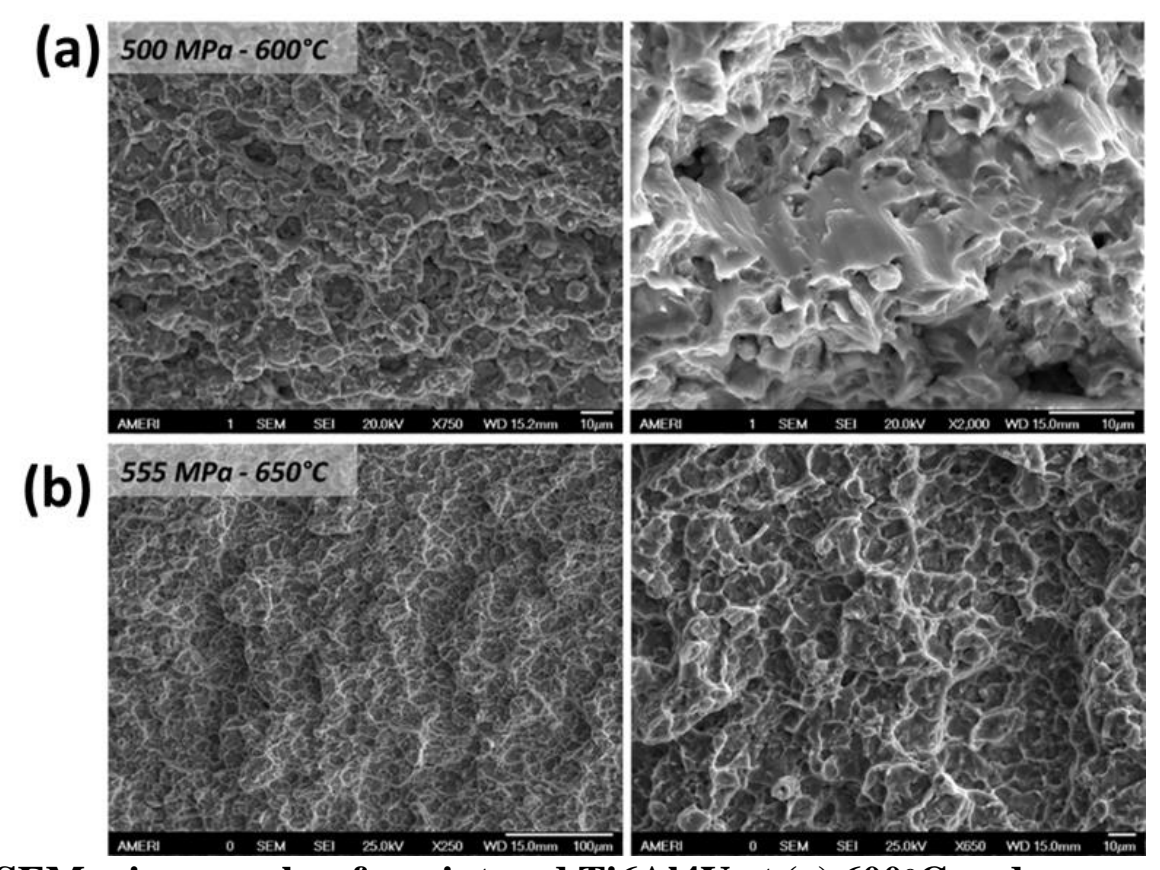

Figure 32 SEM micrographs of as-sintered Ti6AI4V at (a) $600^{\circ} \mathrm{C}$ and pressures of 555 $\mathrm{MPa}$, as well as (b) $650^{\circ} \mathrm{C}$ and $555 \mathrm{MPa}$. Improved interparticle interactions showed near full densification of the Ti alloy.

Evaluating the microstructural evolution of the as-sintered Ti6Al4V specimens is of crucial importance in determining the morphological characteristics that will influence the mechanical performance of the alloy. Figure 33 shows the polished and etched surface of Ti6Al4V specimens sintered using sintering conditions described above. Low sintering temperatures used between $600-650^{\circ} \mathrm{C}$ and high pressures between $300-555 \mathrm{MPa}$ have shown to result in equiaxed globular microstructures. Where the recrystallization of $\mathrm{Ti}$ during sintering is partial, inhibiting the precipitation of large $\beta$-phase deposits. Sintering of $\alpha-\beta$ alloys near the $\beta$-transus temperature is known to result in increased $\beta$-phase concentrations and microstructures dominated by elongated $\alpha$ grains with residual $\beta$-phase along the grain boundaries. Thus, Figure 33 the predominantly globular grains within the microstructure of low temperature sintered Ti6Al4V are of $\alpha$-phase composition with minimal $\beta$-phase. Metals with uniform globular microstructures are often desired over 
elongated grains for load-bearing applications due to their ability to exhibit isotropic mechanical properties. The microstructure of sintered specimens at temperatures of $600^{\circ} \mathrm{C}$ and pressures of $300 \mathrm{MPa}$ show the presence of several voids along particle boundaries signaling the lack of mass transport preventing full densification. While prominent pores are still present, increasing temperatures by $650^{\circ} \mathrm{C}$ and pressures to $555 \mathrm{MPa}$ reduces overall porosity in the metal while maintaining the globular microstructure. Grain growth by $\sim 10 \%$ is experienced with minimal increments in pressure and temperature signaling the improved plastic flow and mass transport.

Interestingly, the increase in sintering pressures during sintering results in the formation of shear-like features along the grains of the Ti6Al4V microstructure (Figure 33 $\mathrm{b}$ and c). The observed features are signaling to the presence of activated twin planes during the high pressure assisted sintering and will be discussed in detail in the following section. Achieving near full densification of Ti6Al4V, a metal often subjected to temperature and time exhaustive processes presents a clear advantage to the ultimate goal of this study "manufacturing of Ti-BNNT composites with control interfacial reactions." 

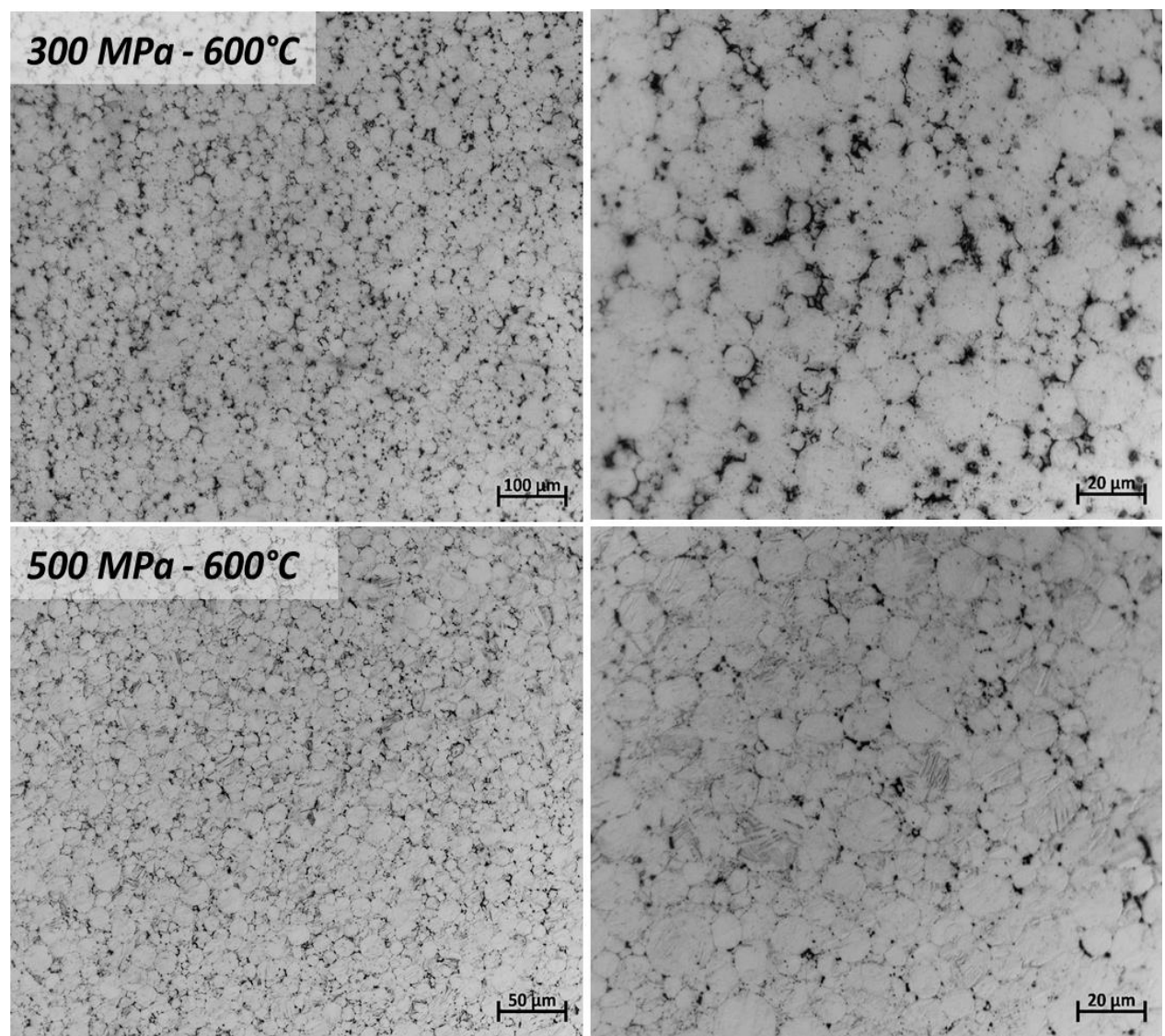

\section{$555 \mathrm{MPa}-650^{\circ} \mathrm{C}$}

$50 \mu \mathrm{m}$

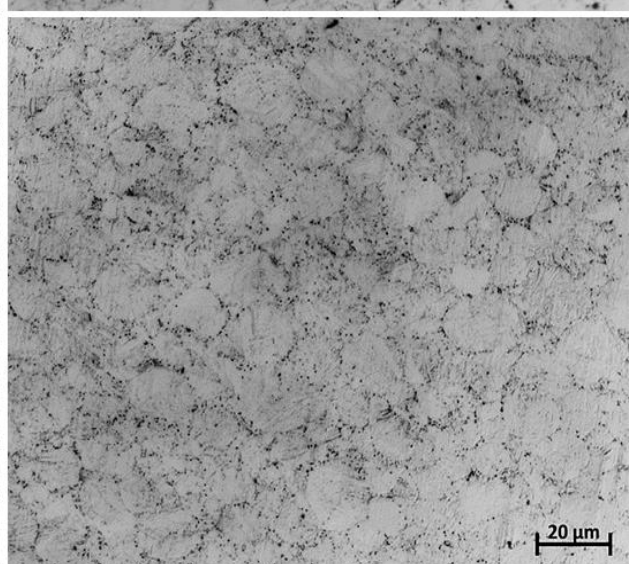

Figure 33 Optical micrographs of the polished and etched surfaces of Ti6Al4V sintered at low-temperature conditions.

\subsubsection{Deformation driven densification:}

The significant decrease in the surface area experienced by Ti particles during high-

pressure sintering is understood as the driving force for the decrease in free energy between solid-void interfaces to soli-solid particles interfaces allowing for near full densifications. 
Mechanisms by which densification is attained were explored by evaluating the crystallographic changes in the alloy. Figure 34 compares the X-ray diffraction pattern of all sintered Ti6A14V specimens at increasing pressures of 300, 500, and $555 \mathrm{MPa}$.

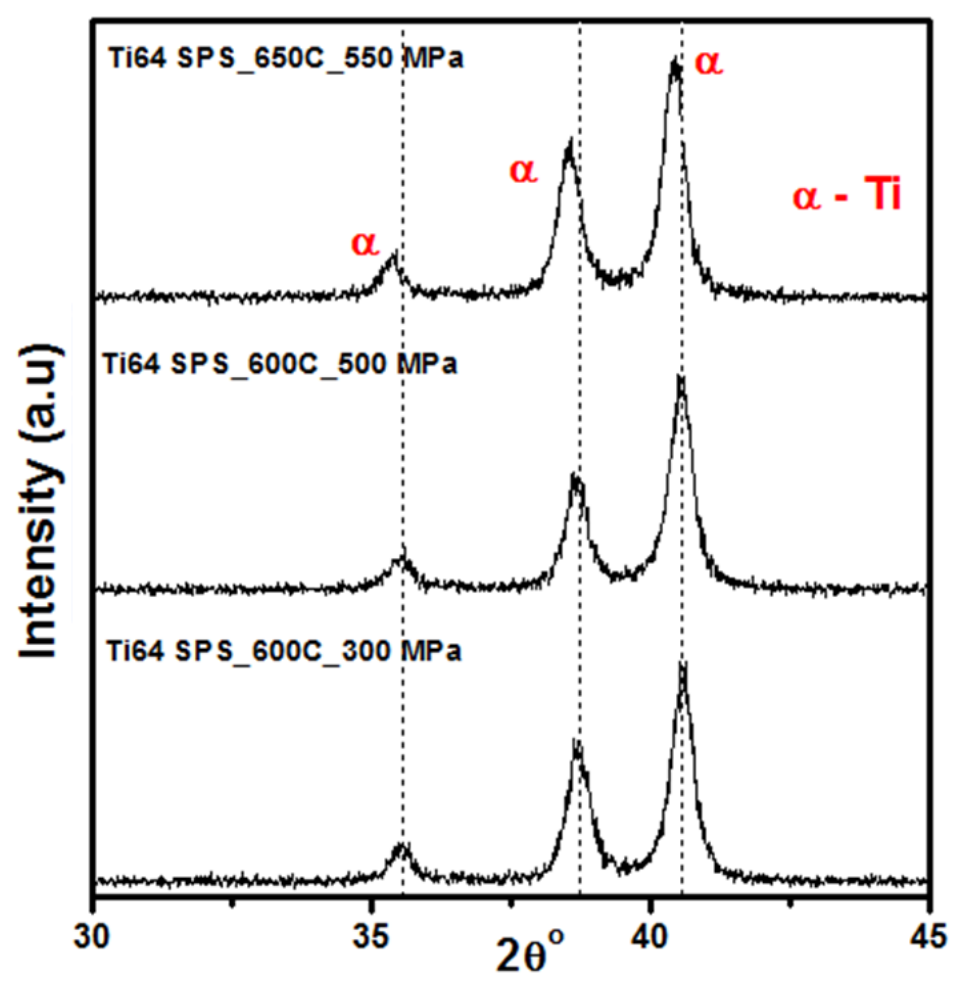

Figure $34 \mathrm{X}$-ray Diffraction pattern of as-sintered Ti6Al4V sample as a function of sintering conditions. Shifting of peaks as a result of high sintering pressures are observed.

Primary peaks corresponding to $\alpha$-phase of the Ti alloy were characterized. No peaks corresponding to the $\beta$ - phase were identified and represent their extremely low concentration $(<9 \%)$ within the sintered alloy. A shift experienced by all major peaks of $\alpha$ Ti towards lower $2 \theta$ angles signaled to distortions in the crystal lattice. Prominent peak shifts are observed in the specimens sintered at highest pressure (555MPa) as compared to 300MPa. The shift of primary peaks corresponding to (100), (002) and (101) planes 
towards lower diffraction angles correspond to the compressive strain in the crystal lattice as a result of high sintering pressures exerted. The degree of deformation experienced by the lattice was evaluated using crystallite size and residual micro-strains. Analysis of the XRD pattern allows us to explore the significance of the mild peak broadening experienced with increasing sintering pressures. Peak broadening in XRD profiles is known to be a result of instrumentation effect, decrease in crystallite size, and lattice strains. In this study, the Williamson-Hall method is used to estimate the average crystallite size and microstrains of the as-sintered Ti6Al4V specimens ${ }^{103}$.

$B \cos \theta=\frac{K \lambda}{D}+\eta \sin \theta$

Where $B$ corresponds to the full width at half maximum (FWHM) obtained from fitting of the experimental XRD curve with $\mathrm{R}^{2}>90$ representing the good fit of the data, $D$ as the average crystallite size, $\eta$ being microstrain (lattice distortions), and $\lambda$ is the wavelength of the $\mathrm{Cu}-\mathrm{K} \alpha$ source $\sim 0.15 \mathrm{~nm}$. The model assumes isotropic nature, in which uniform strains across crystallographic directions is the same and comparison of the curve to a linear curve of the form $y=b+m x$ allows extraction of crystallite size (intercept) and microstrain (slope). Table 6 summarizes the results obtained by the analysis of experimentally obtained XRD patterns. A slight increase in the average crystallite size of specimens sintered at $600^{\circ} \mathrm{C}$ with pressures varying from 300 to $500 \mathrm{MPa}$ signal to the low thermal energy supplied to contribute to grain growth. The opposite is observed in samples sintered at $650^{\circ} \mathrm{C}$ and pressures of $555 \mathrm{MPa}$, where the increase in internal energy of the grains permits their growth by $2 \times$ that of specimens sintered at $600^{\circ} \mathrm{C}$. 
Moreover, compressive strain induced by high sintering pressures is observed to increase microstrains. The significant increase (up to $76 \%$ ) in lattice strain of samples sintered at pressures of 500 and $555 \mathrm{MPa}$ is attributed to the larger number of defects introduced in the microstructure. An increase in volume defects such as dislocations and twins observed in both samples tends to exert lattice strains in the crystal ${ }^{103}$. Table 6 Summary of computed average crystallite size and microstrain using
Williamson-Hall method.

\begin{tabular}{ccc}
\hline Sample & $\begin{array}{c}\text { Average crystallite size } \\
(\mathbf{n m})\end{array}$ & Microstrain \\
\hline $600^{\circ} \mathrm{C}-300 \mathrm{MPa}$ & 46.31 & $1.66 \times 10^{-3}$ \\
\hline $600^{\circ} \mathrm{C}-500 \mathrm{MPa}$ & 55.62 & $2.05 \times 10^{-3}$ \\
\hline $650^{\circ} \mathrm{C}-555 \mathrm{MPa}$ & 110.83 & $2.86 \times 10^{-3}$ \\
\hline
\end{tabular}

The role of crystal lattice defects induced by high sintering pressures in the restriction of dislocation movement and strengthening of the metal is evidenced by performing transmission electron microscopy (TEM) of the specimens sintered at $650^{\circ} \mathrm{C}$ 555MPa. The mechanism by which a metal experiences plastic deformations are commonly understood to be driven by dislocation movement. Upon experiencing increasing stresses, dislocations are continuously generated in the metal and begin to migrate towards pile-up zones ${ }^{104}$. Grain boundaries are the most commonly available barriers to restrict dislocation movements. Hence, the pile-up of dislocations near grain boundaries requires higher stresses for deformation to occur. The sudden increase in dislocation density often experienced would fail a typical metal. The activation of twins in 
the Ti6A14V specimen sintered at low temperatures and high pressures extends the strengthening of the metal by introducing an additional barrier for dislocations to move $\mathrm{e}^{105,106}$. The presence of twins in the microstructure of Ti6Al4V not only represent a strengthening of the metal but also are presumed to improve the ductility.

Figure 35 shows a TEM micrograph of the sintered Ti6Al4V at temperatures of $650^{\circ} \mathrm{C}$ and sintering pressures of $555 \mathrm{MPa}$. In which, the dense dislocation microstructure can be evidenced as described by optical investigation and XRD analysis. The presence of slip bands with dislocations trapped within the boundaries of the bands can be observed. The activation of slip planes in Ti6Al4V alloys with $\alpha$ rich phases has demonstrated to be dependent on the critical resolve shear stress ${ }^{105,106}$. Exceeding the prescribed shearing stress would indicate the initiation of a slip in the alloy. Critical resolve shear stresses for slips to occur within the prismatic and basal <a> direction of the HCP grains is of $340 \mathrm{MPa}$, while that for pyramidal is of $680 \mathrm{MPa}^{105,106}$. Therefore, it can be presumed that the slip bands observed in Figure 35 belong to either basal, or prismatic <a>, for which the yield stress has been far exceed by the sintering pressure (555 MPa). The critical resolve shear stress is highly dependent on temperature and should further reinforce the proposed mechanism of activation ${ }^{105,106}$. 

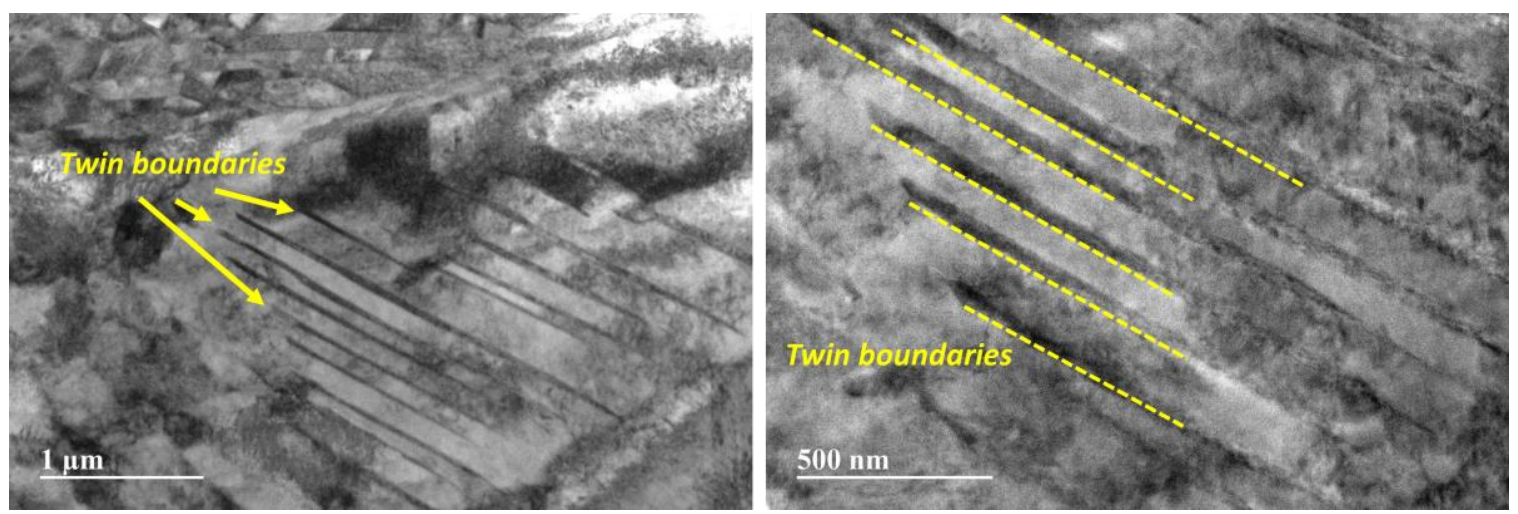

Figure 35 Transmission electron micrograph of Ti6Al4V specimen sintered at $750^{\circ} \mathrm{C}$ and $555 \mathrm{MPa}$ showing slip bands and twin boundaries as hosts for dislocations.

The presence of twins in the microstructure of the Ti and ultimately the Ti-BNNT composites are considered beneficial to strengthening. Twin boundaries in the alloy can be considered as added sources for dislocations upon experiencing deformations. Interactions between dislocation and twin boundaries could lead to an increase in stresses required for plastic deformation to occur due to their ability to pin dislocations. Thus, considering them as a secondary zone for dislocations to pile-up in addition to grain boundaries.

\subsection{High-Temperature sintering of Ti6Al4V}

In light of the achieved success in sintering near fully dense Ti6Al4V specimens by sintering at low temperatures $\left(650^{\circ} \mathrm{C}\right)$, sintering of the alloy was also performed at high temperatures to describe its potential in the manufacturing of Ti-BNNT composites. Although sintering temperatures of $950^{\circ} \mathrm{C}$ are above the oxidation temperature of BNNTs $\left(900^{\circ} \mathrm{C}\right.$ in the air), the rapid densification provided by the SPS technique could prove beneficial to restricting the kinetics of reactions between the nanotubes and the matrix.

Figure 36 a compares the densification curves of both low temperature $\left(650^{\circ} \mathrm{C}\right.$ $555 \mathrm{MPa})$ and high temperature $\left(950^{\circ} \mathrm{C}-60 \mathrm{MPa}\right)$ sintering of the alloy as a function temperature. Sintering at significantly higher temperatures has shown to result in similar 
densification ( $\sim 98.6 \%)$ as that achieved by using low-temperature regimes ( $\sim 98.9 \%)$. This observation further strengthens the potential of the low temperature sintering technique described in the previous section. Similarly, as it was detailed in the low-temperature sintering of the alloy, three regimes are identified for the densification of the alloy. However, it is clear from the curve that the first regime is highly dependent on the sintering pressure used. Sintering pressures of $60 \mathrm{MPa}$ result in merely $62.5 \%$ packing as compared to the almost $80 \%$ achieved by pressures of 555MPa. This clear variation has a collateral effect with the secondary regime $\left(300^{\circ} \mathrm{C}-600^{\circ} \mathrm{C}\right)$, which is observed to be extended by at least $100^{\circ} \mathrm{C}$ in the absence of high pressure. In which, surface diffusion occurs, and minor densification takes place, while necking via transferring of Ti atoms occurs at the particleparticle interface. Finally, densification of the alloy by lattice diffusion takes place at a temperature higher than $600^{\circ} \mathrm{C}$. The steep rise in densification as a function of temperature until reaching the maximum sintering temperature prescribed at $950^{\circ} \mathrm{C}$ characterizes the diffusion and recrystallization of grains occurring. Recrystallization effects are shown by the resulting microstructure with elongated $\alpha$-grains and residual $\beta$-phase within grain boundaries. Upon cooling of the alloy, transformation of $\beta$ to $\alpha$-phase takes place by the nucleation of $\alpha$-phase within transformed $\beta$, giving rise to the plate-like lamellar $\alpha-\beta$ colonies. Also, primary $\alpha$-grains with elongated morphology interface with residual $\beta$ phases minimizing interfacial energy due to its difference in chemical composition ( $\alpha$-Alrich, $\beta-\mathrm{V}$ rich). Consideration of the fracture surface of Ti6Al4V sintered at $950^{\circ} \mathrm{C}$ resolves largely ductile dimples characterizing the increased ductility as a result of higher ductile $\beta$ phase present in the specimen. 

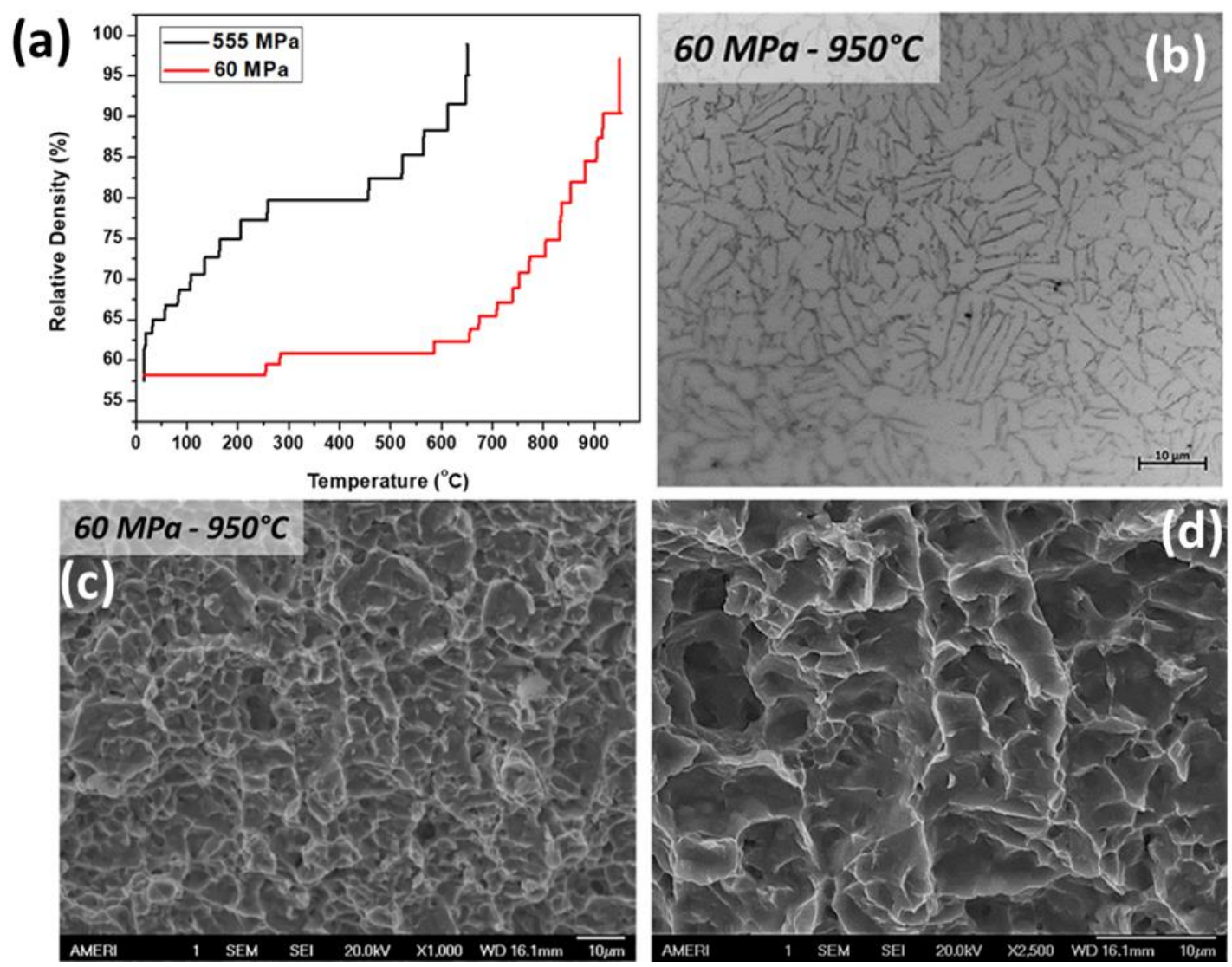

Figure 36 (a) Densification curves comparing sintering mechanisms of hightemperature sintering and low-temperature sintering of Ti6Al4V. (b) Optical micrograph of the etched and polished surface of the alloy sintered at $950^{\circ} \mathrm{C}$ with characteristic elongated $\alpha$-grains. (c) and (d) fracture surface of the alloy sintered at high temperatures showing ductile dimples.

\subsection{Low vs. High-temperature sintering of the alloy}

In the sintering of Ti6Al4V, previously explored sintering routes have shown to involve high temperatures and time-consuming processes which could represent a challenge in the manufacturing of Ti reinforced composites. Owing to the high reactivity of the metal to the BNNTs at elevated temperatures, one of the objectives of this study is to present suitable manufacturing of Ti-BNNT with minimal reaction products. To address the thermodynamic challenge, low-temperature sintering of the alloy is introduced. The proven potential of the technique yielded similar densifications of $98.9 \%$ at $650^{\circ} \mathrm{C}$ as 
compared to commonly used high-temperature sintering conditions. The potential of the technique lies in the sintering temperatures implemented been at least $30 \%$ below temperatures reported for the oxidation of BNNTs in the air $\left(900^{\circ} \mathrm{C}\right)$. Table 7 outlines the advantages and disadvantages presented for both low and high-temperature sintering. Throughout this study, $650^{\circ}$ and $555 \mathrm{MPa}$, as well as $950^{\circ} \mathrm{C}$ and $60 \mathrm{MPa}$ are sintering conditions that will be used for the sintering of Ti-BNNT composites to establish a comparative study. The suitability of both techniques by considering their effect on reaction products and effective strengthening of the composite will be performed.

Table 7 Comparison between low and high-temperature sintering of Ti6Al4V alloys and their contribution to the manufacturing of composites

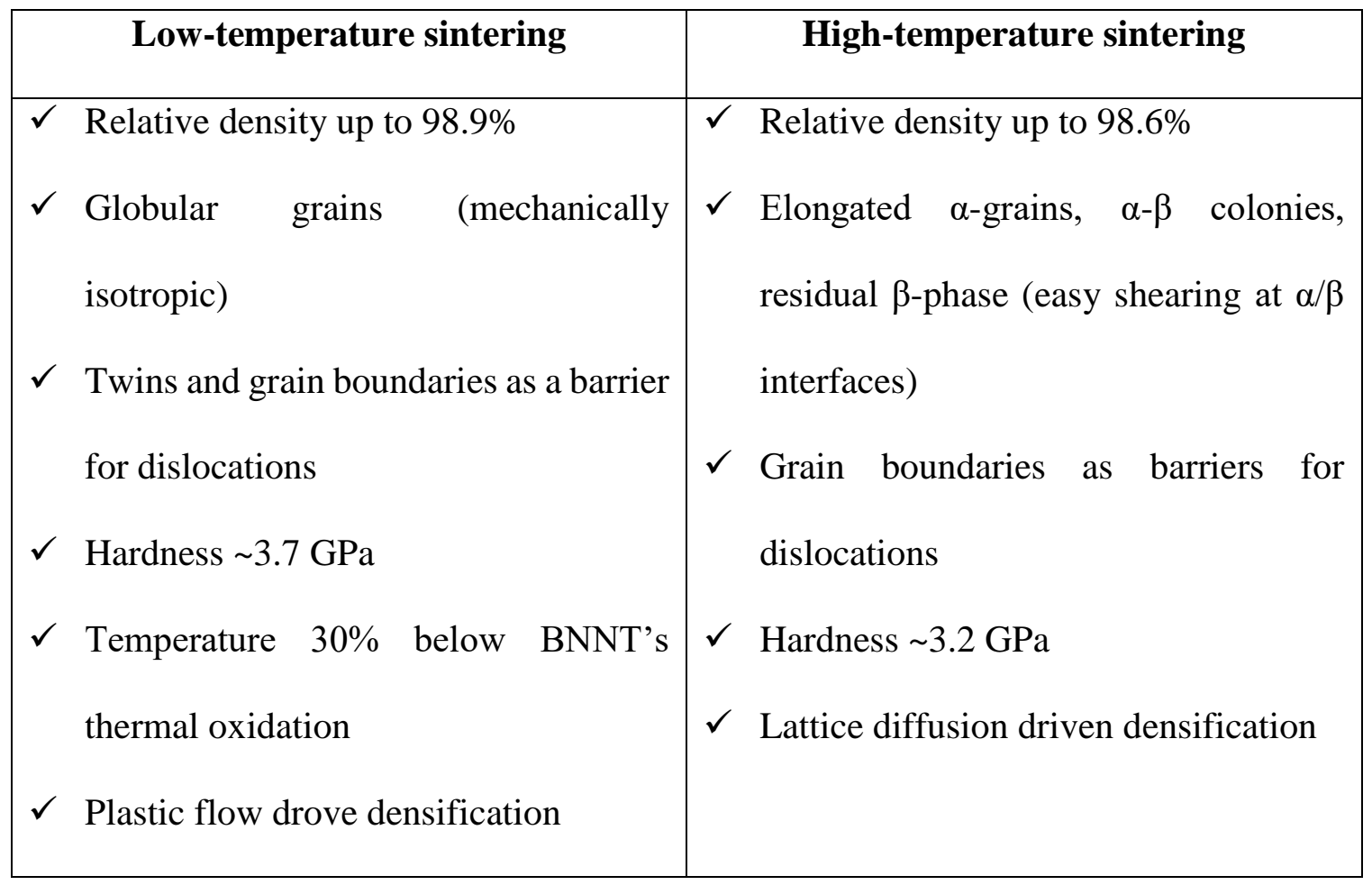




\section{Consolidation of Ti-BNNT composites}

Sintering conditions for the BNNT reinforced Titanium composites were determined as follows:

$\checkmark$ Low-temperature sintering: $650^{\circ} \mathrm{C}-555 \mathrm{MPa}$, chosen based on near full densification achieved in the sintering of pure Ti6Al4V.

$\checkmark$ High-temperature sintering: $950^{\circ} \mathrm{C}-60 \mathrm{MPa}$, temperatures below the $\beta$ transus $\left(\sim 1000^{\circ} \mathrm{C}\right)$ to prevent excessive residual $\beta$-phase in the microstructure.

\subsection{Low vs. High-temperature sintering of Ti-BNNT composites}

The potential of low temperature and high-temperature sintering techniques to manufacture BNNT reinforced Ti composites with controlled interfacial reactions were evaluated. Densification mechanism, thermodynamic limitans and resulting mechanical properties of the composites are discussed.

\subsubsection{Low-temperature sintering of Ti-BNNTs}

\subsubsection{Role of BNNTs in restricting the plastic flow}

Sintering of Ti-BNNT composites with 1 wt.\% BNNT concentration was performed at optimum conditions identified for the sintering of Ti6Al4V within lowtemperature regime $\left(650^{\circ} \mathrm{C}-555 \mathrm{MPa}\right)$. Figure $37 \mathrm{a}$ shows a low magnification SEM micrograph of the fracture surface in the as-sintered Ti-BNNT composite. It is evident by the notable particle boundaries that sintering of the composite has not occurred reached completion given the sintering conditions. Grain boundary (particle-particle interactions) 
are observed as host sites for BNNTs (Figure 37b and c). In which, they can be understood to behave as inhibitors for deformation driven densification at the interfaces. Interfacial interactions between the Ti-matrix and the nanotubes can be observed to be primarily by adhesion forces and not covalent bonding as it has been reported in prior works (Figure 37c). Where the interactions between the BNNTs and the metallic matrix result in limited chemical interactions responsible for strong bonding. The segregation of BNNTs at the grain boundaries without prevalent chemical interactions at the metal/nanotube interface is considered detrimental to the mechanical performance of the composite. Weak van der Waal forces are considered to be governing interactions among BNNTs and those interfacing the metal. In which, increasing stresses promote shearing among agglomerated nanotubes and promote the catastrophic failure of the structures. Although low-temperature sintering conditions of $650^{\circ} \mathrm{C}$ and $555 \mathrm{MPa}$ resulted in the successful densification and sintering of Ti6Al4V, BNNTs were observed to behave as barriers requiring higher activation energies to induce plastic flow during sintering. 

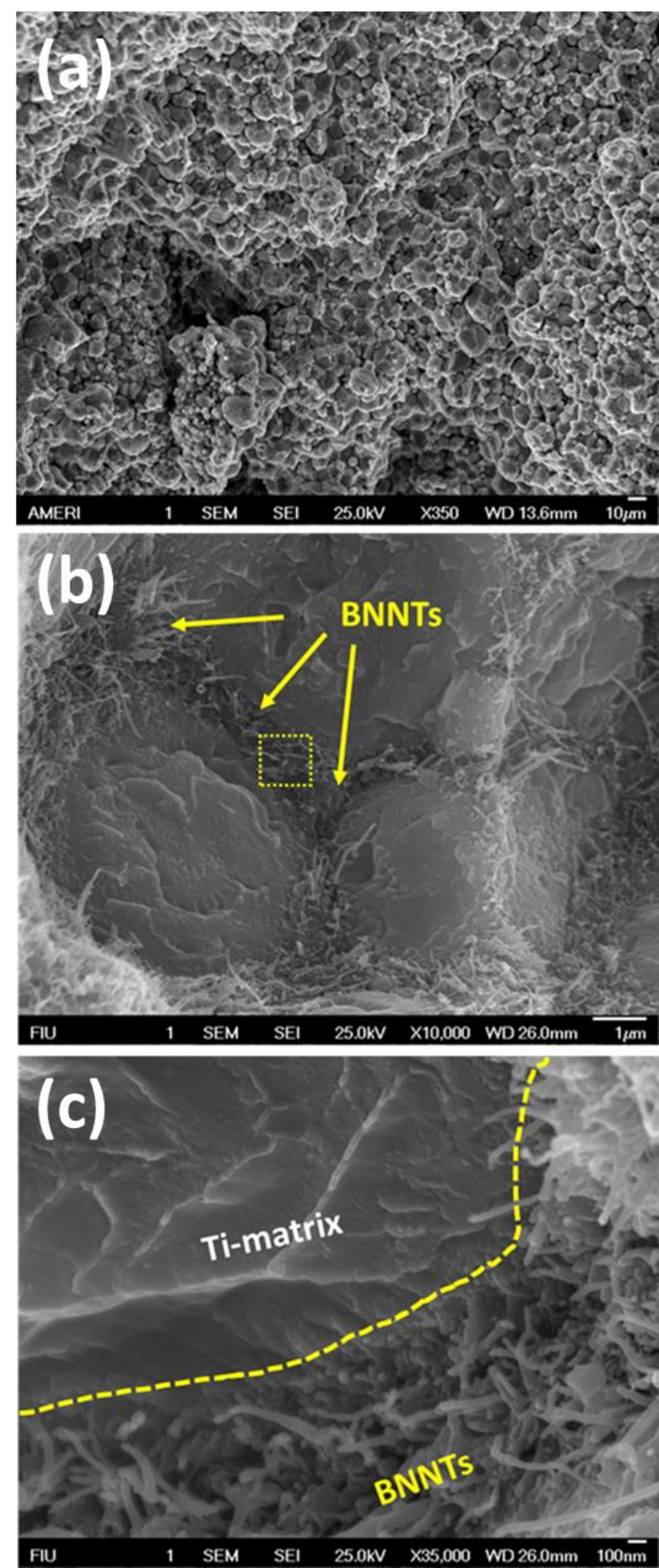

Figure 37 Fracture surface of as-sintered Ti-BNNT composite at $650^{\circ} \mathrm{C}$ and $555 \mathrm{MPa}$ pressures (a) showing poor sintering and particle-particle interactions, (b)restricted plastic flow by BNNTs located at the interface of the Titanium grain boundaries 
Sintering of metals and composites is a process driven by diffusion of atoms. In which, pure elements experience self-diffusion, and the presence of other compounds could introduce vacancy dominated and/or interstitial dominated mechanisms. The diffusion Boron and Nitrogen atoms into the Titanium matrix to form covalent bonds is driven by interstitial diffusion as a result of the small atomic size as compared to $\mathrm{Ti}$ atoms. Dominating reactions expected to occur within the Ti-BNNT system include (referenced from Chapter II):

$2 \mathrm{Ti}+\mathrm{BN}=\mathrm{TiB}_{2}+\mathrm{TiN}$

$\mathrm{Ti}+\mathrm{TiB}_{2}=2 \mathrm{TiB}$

For which, Nitrogen is expected to diffuse into the Ti matrix and form solid solutions and precipitates of $\mathrm{TiB}_{2}$ and $\mathrm{TiB}$ can result from thermodynamically feasible environments. These reactions are of prime importance for the sintering of Ti-BNNT composites, as they will dictate the strength of interfacial interactions. Thus, impacting the sintering of the specimen and mechanical properties.

Given the dependence of densification and sintering of the Ti-BNNT on the formation of reaction products between the matrix and nanotube, Thermo-Calc software was implemented to estimate the Gibs free energy of reactions in the Ti-BN system.

Evaluation of thermodynamic reactions at sintering temperatures of $650^{\circ} \mathrm{C}$ of the Ti-BN system containing 1 wt. $\%$ BNNT, resulted in $\Delta \mathrm{G}=-38.9 \mathrm{~kJ} / \mathrm{mol}$. The required activation energy for the diffusion of Boron into Ti has been reported to be of 38.28 
$\mathrm{kJ} / \mathrm{mol}^{107}$. It is noted that the estimated Gibbs free energy of the system at $650^{\circ} \mathrm{C}$ is within limits for activating diffusion of B into the Ti matrix. However, computed thermochemical reactions estimate this to result in merely 0.098 mass percentage diffusion of B into the largely Ti matrix. It is essential to highlight that the minimal mass percentage of B diffused into $\mathrm{Ti}$ is well below the required composition for precipitation of $\mathrm{TiB}_{2}$ and/or $\mathrm{TiB}$ phases. This is experimentally evidenced in Figure 37c, where interactions between the Ti and BNNTs are weak, and no evidence of $\mathrm{TiB}$ or $\mathrm{TiB}_{2}$ precipitates are observed.

\subsubsection{Improved interfacial interactions at $750^{\circ} \mathrm{C}-555 \mathrm{MPa}$}

In the continuation of this study, sintering conditions ruling the low-temperature sintering of Ti-BNNT composites were chosen as $750^{\circ} \mathrm{C}$ and $555 \mathrm{MPa}$. The increase in thermal energy in combination with high pressures, is expected to improve the interfaces of the Ti-BNNT composites. The fracture surface of the sintered Ti-BNNT composites at $750^{\circ} \mathrm{C}$ and $555 \mathrm{MPa}$ is shown in Figure 38. Dimple morphology within the fracture surface sintered to the improved interactions between powder particles. The improved sintering was also observed by the increased relative densification of $\sim 97.8 \%$. As compared to a pure Ti6A14V sintered at similar conditions which achieved a relative density of $98.4 \%$, the composite shows partial sintering where BNNTs are encountered. Improved metal/nanotube interfaces can be observed in Figure 38b. Higher temperatures induced during sintering in addition to high sintering pressures have allowed improved plastic flow for Ti particles-particles to interact and enhance their interfaces. However, the plastic flow phenomena described is not observed to be uniform across the fracture surface of the 
specimen. Figure 38c shows instances where nanotubes located at the grain boundaries have inhibited diffusion mechanisms from taking place.

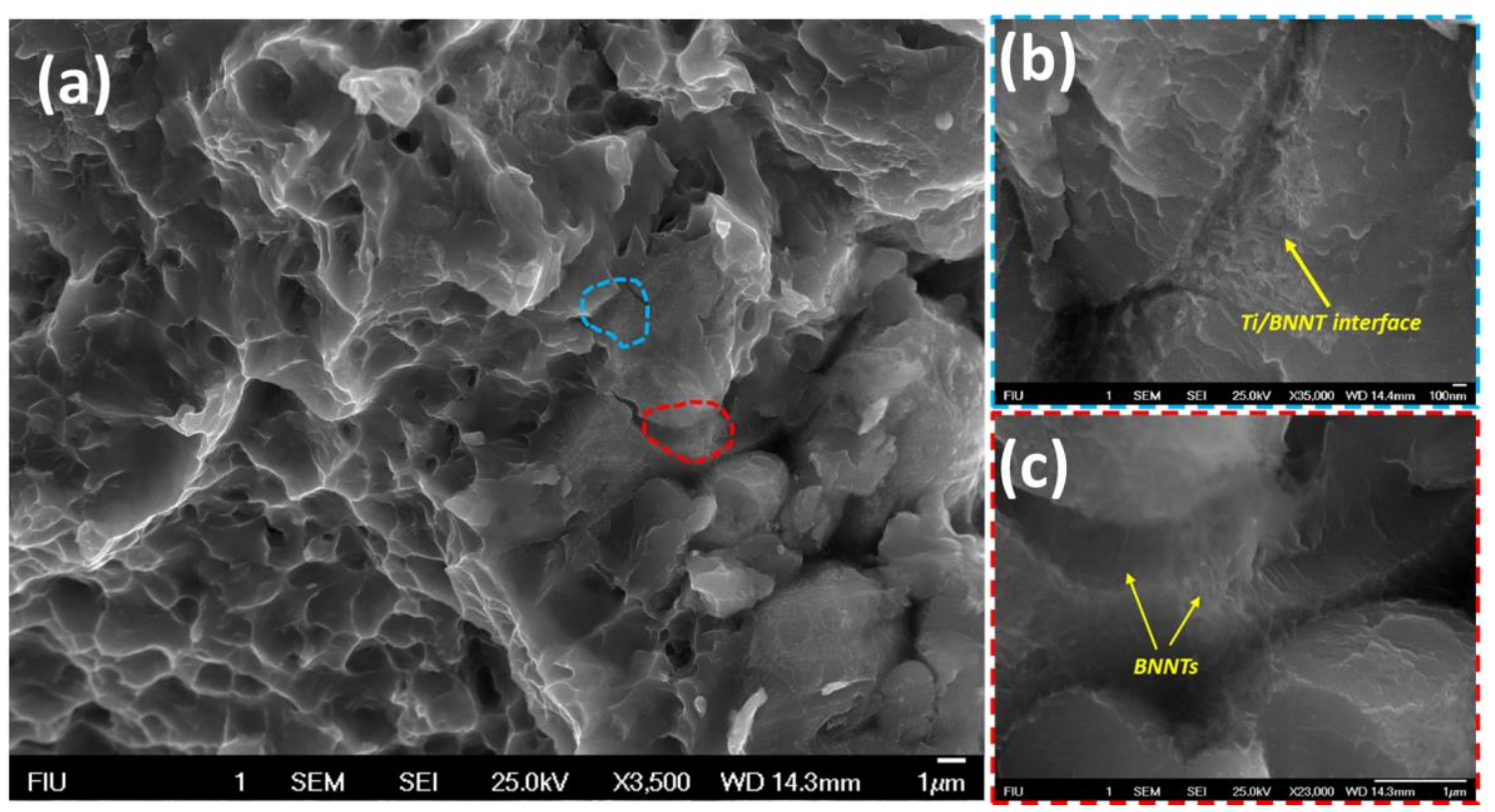

Figure 38 (a) Improved fracture surface of Ti-BNNT composites sintered at $750^{\circ} \mathrm{C}$ and $555 \mathrm{MPa}$ with (b) enhanced interfacial interactions and (c) no reaction products

Evaluation of phases present in the composite sintered at $750^{\circ} \mathrm{C}$ and $555 \mathrm{MPa}$ was performed by analysis of the corresponding XRD pattern. Figure 39 displays a comparison of XRD curves corresponding to pure Ti6Al4V and Ti-BNNT composite sintered at stipulated low-temperature sintering conditions $\left(750^{\circ} \mathrm{C}\right.$ and $\left.555 \mathrm{MPa}\right)$. While the fracture surface showed no evident interfacial reactions in the form of $\mathrm{TiB}$ nanoparticles or $\mathrm{TiB}_{2}$ needles/whiskers, XRD can provide insights on their presence if any. Peaks identified correspond to primarily $\alpha$-Titanium. Close observation of the XRD pattern of the composite within $2 \theta$ diffraction angles of $25-45^{\circ}$ revealed no presence of peaks corresponding to interfacial reactions. Thus, evidencing their minimal concentration within the sintered composite, if any present. 


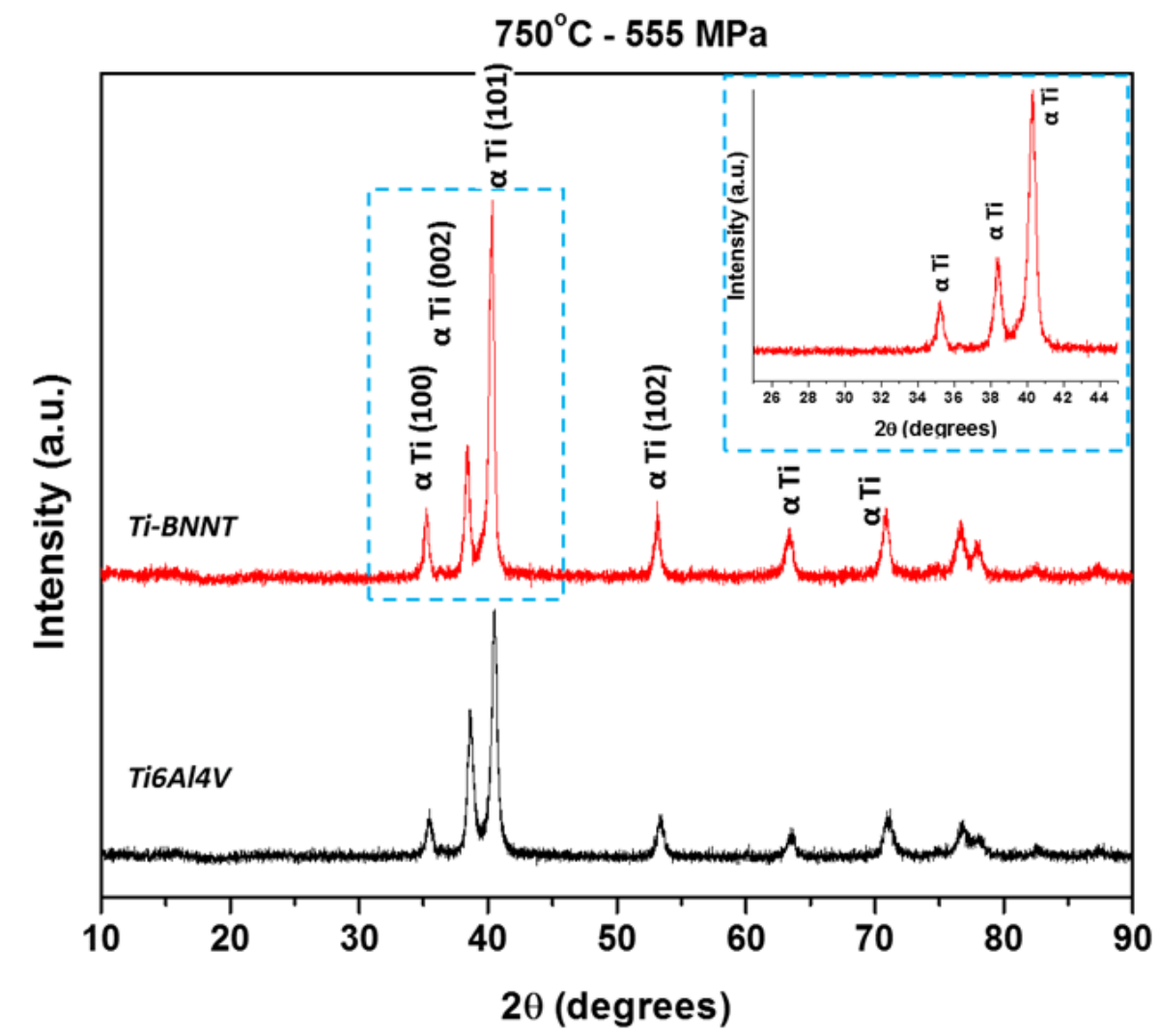

Figure 39 XRD pattern of as-sintered composite and control specimen at $750^{\circ} \mathrm{C}$ and $555 \mathrm{MPa}$ with primary peaks corresponding to $\alpha$-Ti and no reaction phases identified

In a similar fashion to samples sintered at $650^{\circ} \mathrm{C}$, thermodynamically feasible reactions in the Ti-BN system were performed at $750^{\circ} \mathrm{C}$ using Thermo-Calc software. For which, sintering conditions of $750^{\circ} \mathrm{C}$ resulted in Gibs free energies of $\Delta \mathrm{G}=-45.18 \mathrm{~kJ} / \mathrm{mol}$. It is important to highlight that the estimated calculations do not account for pressure induced mechanisms aiding in the diffusion by minimizing interparticle distance. The improved interfacial interactions between the Ti and BNNTs can be deduced to be a combination of the thermally activated diffusion and high pressure aiding the diffusion mechanisms to take place.

Figure 40a depicts the microstructure of the etched surface of the as-sintered TiBNNT at $750^{\circ} \mathrm{C}$. The low temperature sintered specimen is characterized by having 
globular grains with BN rich phases sitting at the grain boundaries. Indication of the phases been BNNT rich and not voids as observed in samples sintered at $650^{\circ} \mathrm{C}$ are shown in Figure $40 \mathrm{~b}$ and $\mathrm{c}$, where the survival of nanotubes to high pressures and their improved interactions with the matrix are evidenced. The presence of nanotubes at the grain boundaries are responsible for grain growth suppression and restriction to deformations. Thus, a microstructure as that displayed in Figure 40 can be considered as beneficial to the strengthening of Ti matrices given improved interfacial interactions.
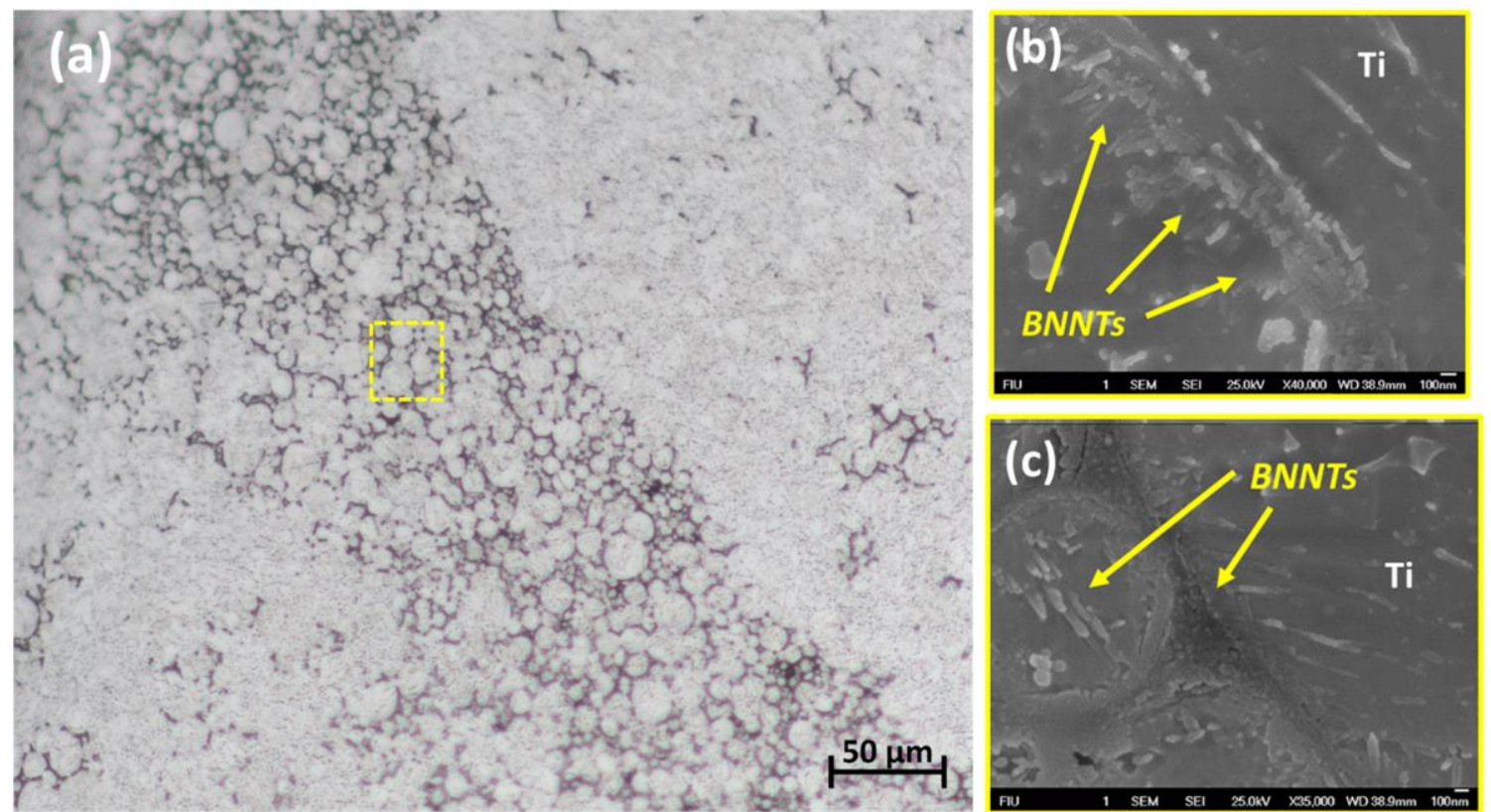

Figure 40 (a) Optical micrograph of the polished and etched surface of the Ti-BNNT composite sintered at low temperatures and high pressures with globular morphologies. (b) and (c) Presence of BNNTs across grain boundaries without morphological changes.

High-resolution transmission electron microscopy of the Ti-BNNT sample uncovered the presence of twin boundaries in its microstructure (Figure 41). The presence of twins within the microstructure is a significant defect activated by high-pressure sintering of the Ti6Al4V. The large presence of twins was also evidenced in the pure Ti metal described in the previous section. The significance of twins in the microstructure of 
the composite lies in their ability to act as pile-up zones for dislocations. In this manner, preventing dislocation movement and requiring higher stresses to induce deformations. In addition to the strengthening mechanism intrinsic to the microstructure of low temperature sintered alloy, BNNTs have reported their strengthening effect in the form of dislocation barriers. Therefore, the strengthening of the composite can be considered to have three main mechanisms by which dislocation movement will be restricted: Grain boundaries, twin boundaries, and BNNT reinforcements.
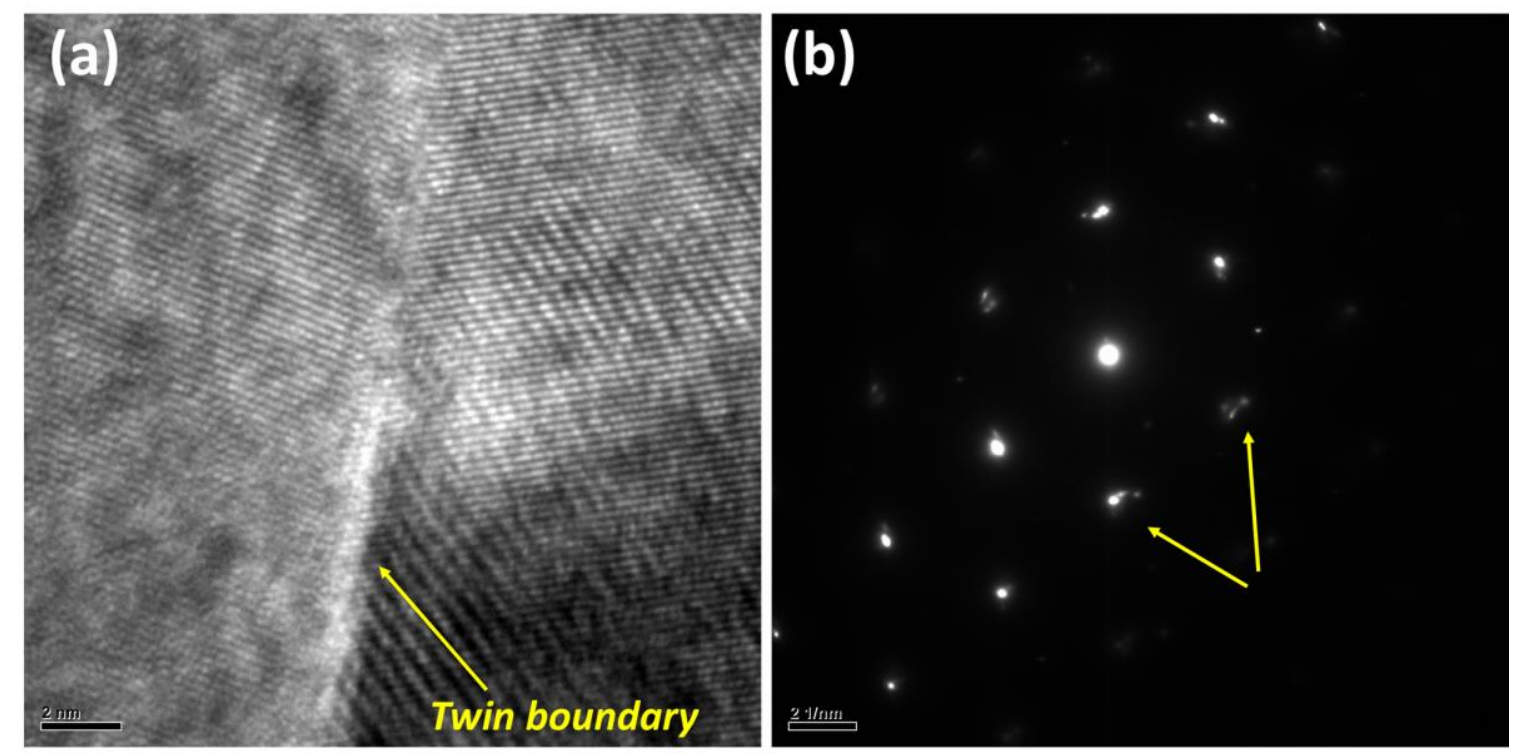

Figure 41 (a) High resolution (HR) Transmission electron micrograph of the TiBNNT composites sintered at $750^{\circ} \mathrm{C}$ and $555 \mathrm{MPa}$ with evidence of twin boundaries (b) corresponding SAED pattern showing diffraction planes of twins

\subsubsection{High-temperature sintering of Ti-BNNTs}

\subsubsection{Phases present and BNNT survival}

Sintering at elevated temperatures of $950^{\circ} \mathrm{C}$ and pressures of $60 \mathrm{MPa}$ was performed to evaluate the ability of BNNTs to resist high-temperature processes. The challenge concerning the processing of BNNT reinforced metallic composites lies in the 
high reactivity of the metallic matrices with the nanotubes at elevated temperatures. Exposure of the nanotubes to higher temperatures has shown to result in the formation of undesirable reaction phases of brittle nature. Spark plasma sintering has shown potential over the manufacturing of BNNT reinforced Al matrices. Where reaction phases with thickness below $20 \mathrm{~nm}$ are found to interface the nanotubes and result in strong interactions with the $\mathrm{Al}$ matrix ${ }^{54}$. Thus, the control of interfacial reactions is of prime importance. This study implements SPS as a processing technique due to its ability to reach structures with near full densification in periods below $30 \mathrm{~min}$. In this manner, aiming to restrict the kinetics of reactions occurring at the interface of the Ti and BNNTs. Figure 42 shows XRD patterns for pure Ti6Al4V and Ti-BNNT composite sintered at $950^{\circ} \mathrm{C}$. Analysis of the curves demonstrates the indexing of primary $\alpha$-Ti phases with the absence of h-BN or Ti$\mathrm{BN}$ reaction phases. The absence of peaks corresponding to $\mathrm{h}-\mathrm{BN}$ and potential reaction phases are attributed to the detection limit of the equipment to detect small concentrations within the composite. Hence, it can be deduced that sintering of the composite even at temperatures of $950^{\circ} \mathrm{C}$ has resulted in minimal reaction products. The Ti-BNNT composite is characterized by exhibiting $97.5 \%$ relative density as compared to pure Ti6Al4V sintered at similar conditions which achieve relative densities of $98.7 \%$. 


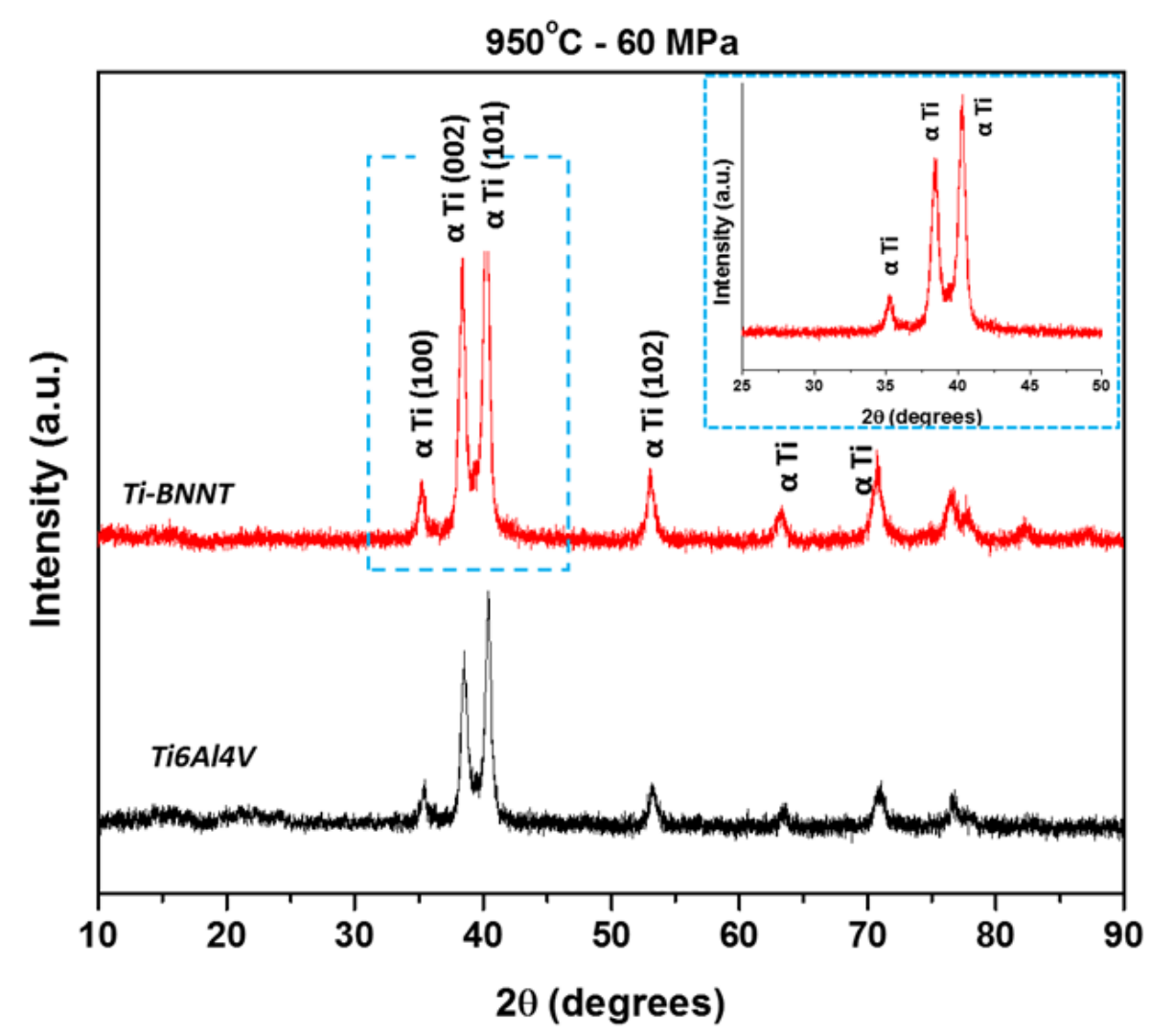

Figure $42 \mathrm{X}$-ray diffraction pattern of the as-sintered Ti-BNNT composite at high temperatures of $950^{\circ} \mathrm{C}$ with no presence of reacted phases in the pattern due to small quantity if any.

Further evaluation of the sintered composite was performed by scanning electron microscopy. Figure 43 shows the fracture surface of the Ti-BNNT composite after been subjected to sintering temperatures of $950^{\circ} \mathrm{C}$. The fracture surface of the composite reveals predominantly dimple structures characterizing the interactions between Ti grains and signaling the ductility of the composite. Sintering at temperatures of $950^{\circ} \mathrm{C}$ and above are known to result in the recrystallization of Titanium grains, in which transformed $\beta$-phases during sintering process serve as nucleation sites for the growth of secondary $\alpha$-phases. The growth of secondary phases within the $\beta$-phases gives rise to $\alpha-\beta$ colonies with platelike structures. Also, grain boundaries surrounding primary $\alpha$-grains are known to provide increased ductility to the Ti matrix. 
Figure 43a shows the present of voids outlined by the dashed yellow marker. A closer look at these regions demonstrated the presence of long nanotubes with smooth surface proving their survival to elevated sintering temperatures and pressure. Well interfaced BNNTs within the Ti-matrix can be observed in Figure 43c. In which, the combination of sharp needle structures and smooth nanotubes represent the interfacial control reactions. Sharp needle structures, as shown in Figure 43c, represent TiB reaction products. As it was shown in previous sintered specimens at low temperatures $\left(<750^{\circ} \mathrm{C}\right)$ and reported in the literature, the lack of interfacial interactions in the form of reaction products is considered detrimental to the reinforcement of composites in the ease of shearing of weakly interfaced BNNTs/metal matrix. On the other hand, the excess of interfacial reactions (thickness $>100 \mathrm{~nm}$ ) represents the complete reaction of nanotubes and result in brittle structures. 


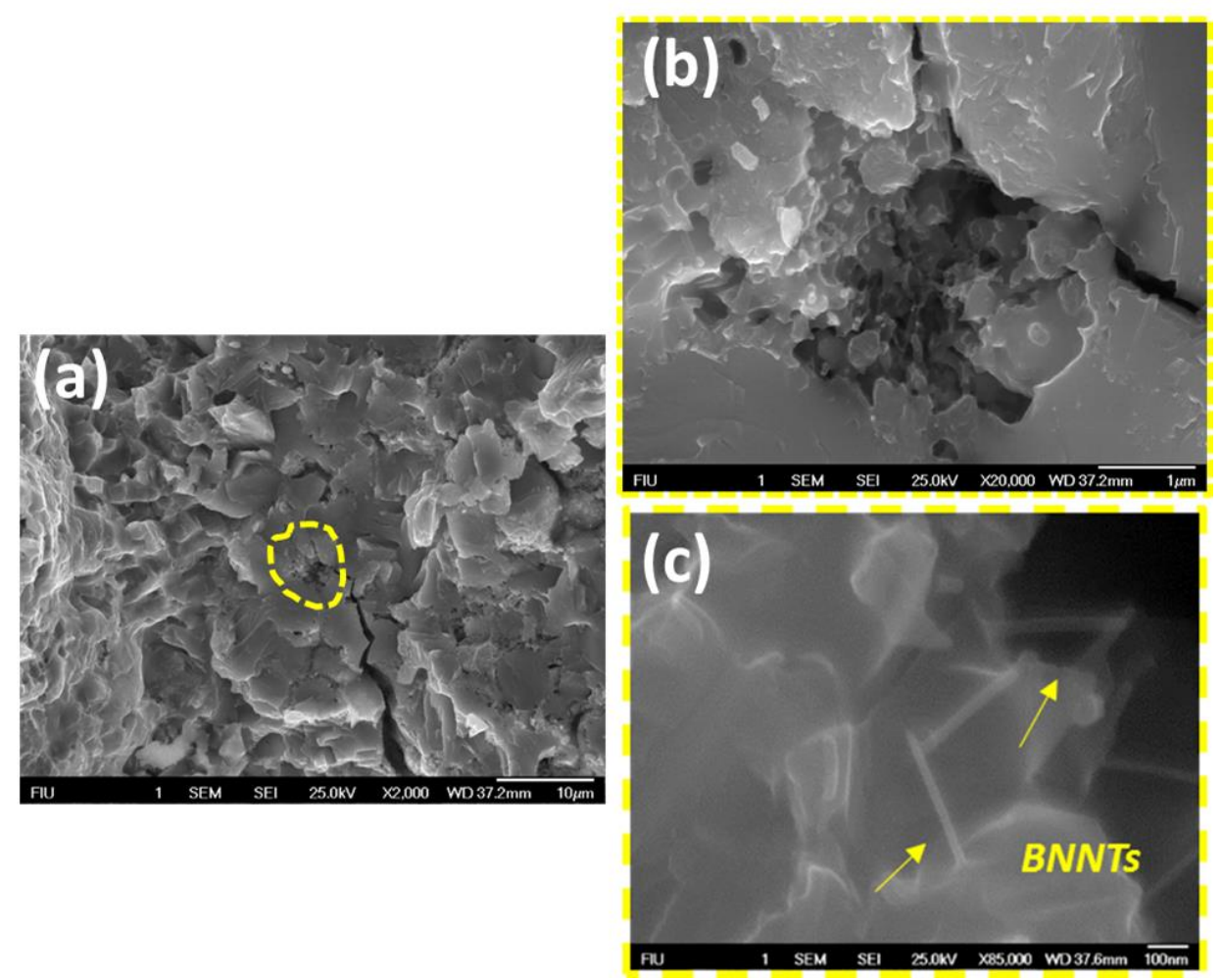

Figure 43 Fracture surface of the (a) Ti-BNNT composites sintered at hightemperature conditions, (b) and (c) survived BNNTs with some partial reactions of straight needle features.

\subsubsection{Thermodynamics of reactions}

The thermodynamic transformations in the Ti-BN system have been explored previously in the manufacturing of in-situ $\mathrm{TiB}$ reinforcements within a $\mathrm{Ti}$ matrix. While the mechanisms by which the phase reactions occur are still valid to this study, the morphology of the reactants (nanotubes) will influence the transformations experienced.

Figure 44 demonstrates the partial and non-uniform reactions that the nanotubes have experienced as a result of exposure to elevated sintering temperatures. Needle-like structures characterizing the $\mathrm{TiB}$ phase reactions are predominantly found as compared to other reactions (i.e., $\mathrm{TiB}_{2}$ ). Although the $\mathrm{TiB}_{2}$ reaction has been found as the more thermodynamically feasible reaction with Gibbs free energy of $\Delta \mathrm{G}=-292.88 \mathrm{~kJ} / \mathrm{mol}$ as 
compared to the TiB phase $(\Delta \mathrm{G}=167.36 \mathrm{~kJ} / \mathrm{mol})$ at temperatures of $950^{\circ} \mathrm{C}$, the kinetics of reactions dominate the transformations.
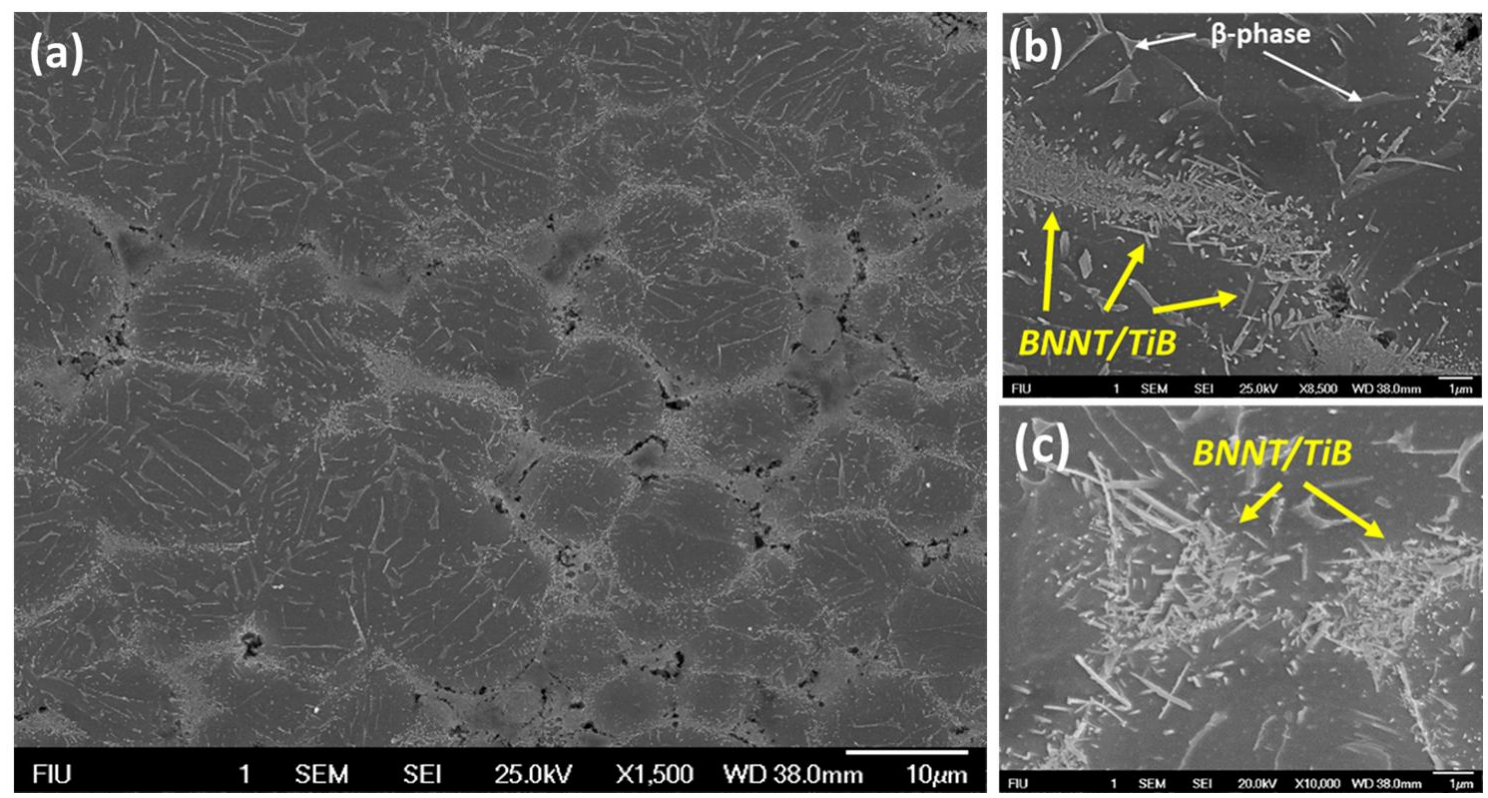

Figure 44 (a) Polished and etched the surface of the high temperature sintered TiBNNT composite showing microstructural features. (b) and (c) Strong interactions between the BNNTs and the matrix with some reacted TiB phases near the grain boundaries.

The reactions can be described by the initial diffusion of Nitrogen atoms into the Ti matrix. The high solubility of Nitrogen atoms within interstitials of Ti atoms results in the formation of solid solutions (TiN) and give rise to the diffusion of Boron atoms within the walls of BNNTs ${ }^{47,48}$. Evidence of TiN solid solution is shown in Figure 45a, in which a line scan shows the phase concentration of Ti and Nitrogen along with the matrix of the composite. 

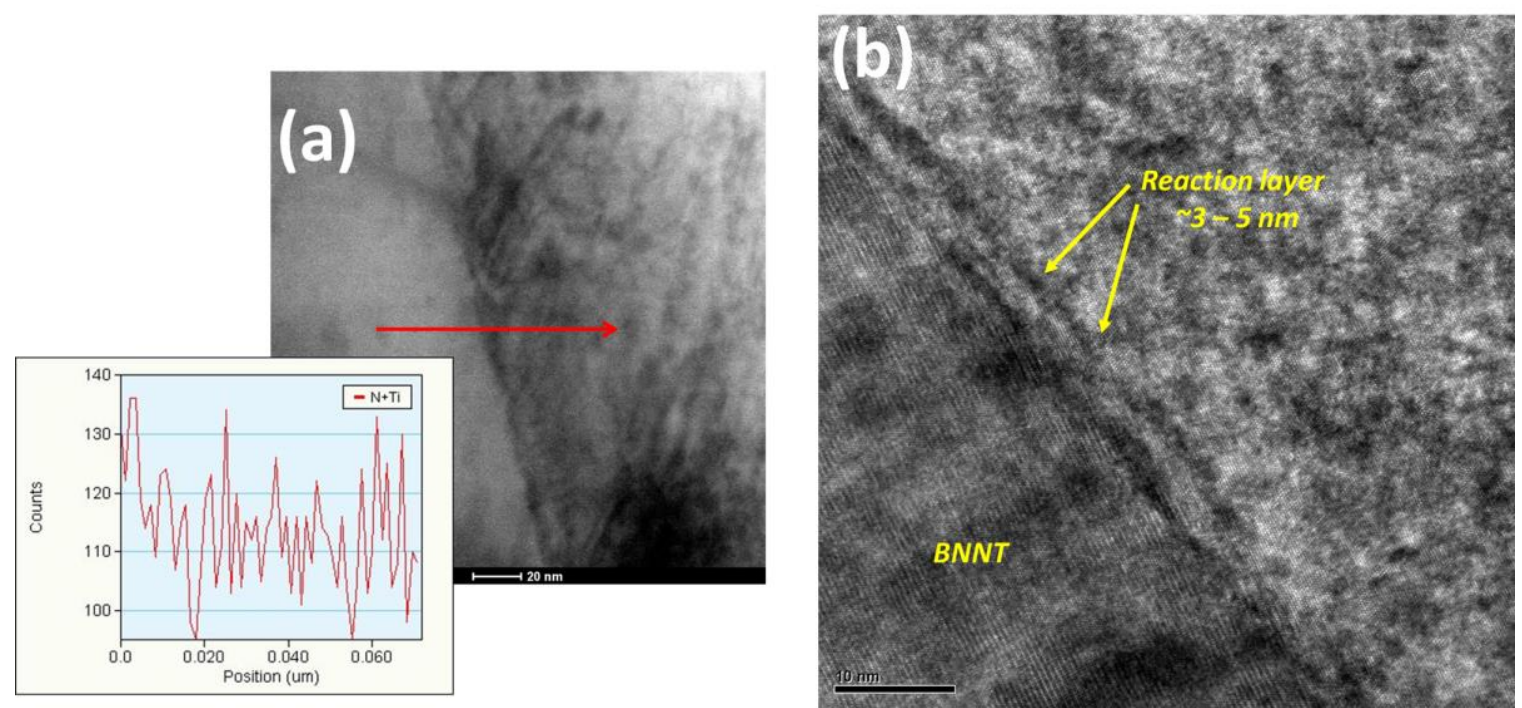

Figure 45 (a) EDX line mapping of the surface of the Ti-BNNT composite sintered at high temperatures, with evidence of $\mathrm{TiN}$ as a solid solution. (b) The interface of nanotube walls with $\mathrm{d}$-spacing of $\sim 0.3-0.4 \mathrm{~nm}$ and potential reaction layer on its surface.

Diffusion of Boron is the predominant in the Ti matrix. Bhuiyan et al. ${ }^{48}$ describe the process by which precipitation of $\mathrm{TiB}_{2}$ and $\mathrm{TiB}$ phases occurs. Owing to the initially high localized concentration of Boron at the interface between the nanotube and the $\mathrm{Ti}$ matrix, nucleation of the most thermodynamically feasible phase (i.e., $\mathrm{TiB}_{2}$ ) occurs in the form of precipitates along the surface of the nanotubes ${ }^{47,48}$.

The rapid diffusion of Boron atoms at elevated temperatures is responsible for the decreased in the localized concentration of Boron experienced at the interface of the nanotube and the $\mathrm{Ti}$. Thus, resulting in the subsequent reaction between the nucleated $\mathrm{TiB}_{2}$ phase and the Ti- matrix to form TiB needles by consuming the inner surface of the nanotube $\mathrm{e}^{47,48}$. It is evident Figure $44 \mathrm{~b}$ and $\mathrm{c}$ that the reaction of nanotubes to form TiB needles has been achieved to a certain degree. However, the presence of intact nanotubes well interfaced within the Ti-matrix can be considered as partial reactions. Figure 45b shows a TEM micrograph of the surface of a BNNT wall with d-spacing of $\sim 0.3-0.4 \mathrm{~nm}$ 
characteristic of h-BN, with what appears to be a reaction layer of thicknesses between 3 $-5 \mathrm{~nm}$.

\subsection{Evaluation of mechanical properties of Ti-BNNT composites under compressive loadings}

\subsubsection{Mechanical properties of Ti-BNNT composites under compression}

The mechanical properties of the composite were investigated under compressive forces. Cylindrical structures with diameters of $2 \mathrm{~mm}$ and thicknesses between $3-4 \mathrm{~mm}$ were machined by wire EDM from as-sintered specimens to evaluate the mechanic properties as a function of sintering temperature and BNNT addition in the Ti-matrix. Compression of pure Ti6Al4V (Ti64) and Ti-BNNT composites sintered at $650{ }^{\circ} \mathrm{C}, 750$ ${ }^{\circ} \mathrm{C}$ and $950^{\circ} \mathrm{C}$ were subjected to compressive loads to up to $75 \%$ compression of its height. Table 8 displays a summary of the yield strength, compressive strength, and failure strain for all samples. It should be noted that values of compressive strength and failure strain of pure Ti6Al4V at all conditions were not achieved under compressive strains prescribed $(75 \%)$ and therefore not reported.

Table 8 Summary of compressive properties of Ti-BNNT composites sintered at high and low temperatures

\begin{tabular}{|c|c|c|c|c|c|c|}
\hline & \multicolumn{2}{|c|}{$650^{\circ} \mathrm{C}-555 \mathrm{MPa}$} & \multicolumn{2}{|c|}{$750^{\circ} \mathrm{C}-555 \mathrm{MPa}$} & \multicolumn{2}{|c|}{$950^{\circ} \mathrm{C}-555 \mathrm{MPa}$} \\
\hline & Ti64 & Ti-BNNT & Ti64 & Ti-BNNT & Ti64 & Ti-BNNT \\
\hline $\begin{array}{c}\text { Yield } \\
\text { Strength } \\
(\text { MPa })\end{array}$ & 6.1 & 5.17 & 10.33 & 12.47 & 31.84 & 47.85 \\
\hline $\begin{array}{c}\text { Compressive } \\
\text { strength } \\
(\mathrm{MPa})\end{array}$ & - & $244.2 \pm 20$ & - & $\begin{array}{c}291.9 \pm \\
20.6\end{array}$ & - & $\begin{array}{c}282.3 \pm \\
16.5\end{array}$ \\
\hline $\begin{array}{c}\text { Strain at } \\
\text { break (\%) }\end{array}$ & - & $46 \pm 4$ & - & $66 \pm 2$ & $\begin{array}{c}\text { Buckled } \\
\text { at } 40 \%\end{array}$ & $51.8 \pm 1$ \\
\hline
\end{tabular}


The corresponding stress-strain curve describing the compression behavior of the samples sintered at $750^{\circ} \mathrm{C}$ and $555 \mathrm{MPa}$ are shown in Figure 46a. It is evident that the addition of $1 \mathrm{wt} . \%$ BNNT in the Ti-matrix resulted in $~ 21 \%$ increase of the yield strength of the Ti-matrix.
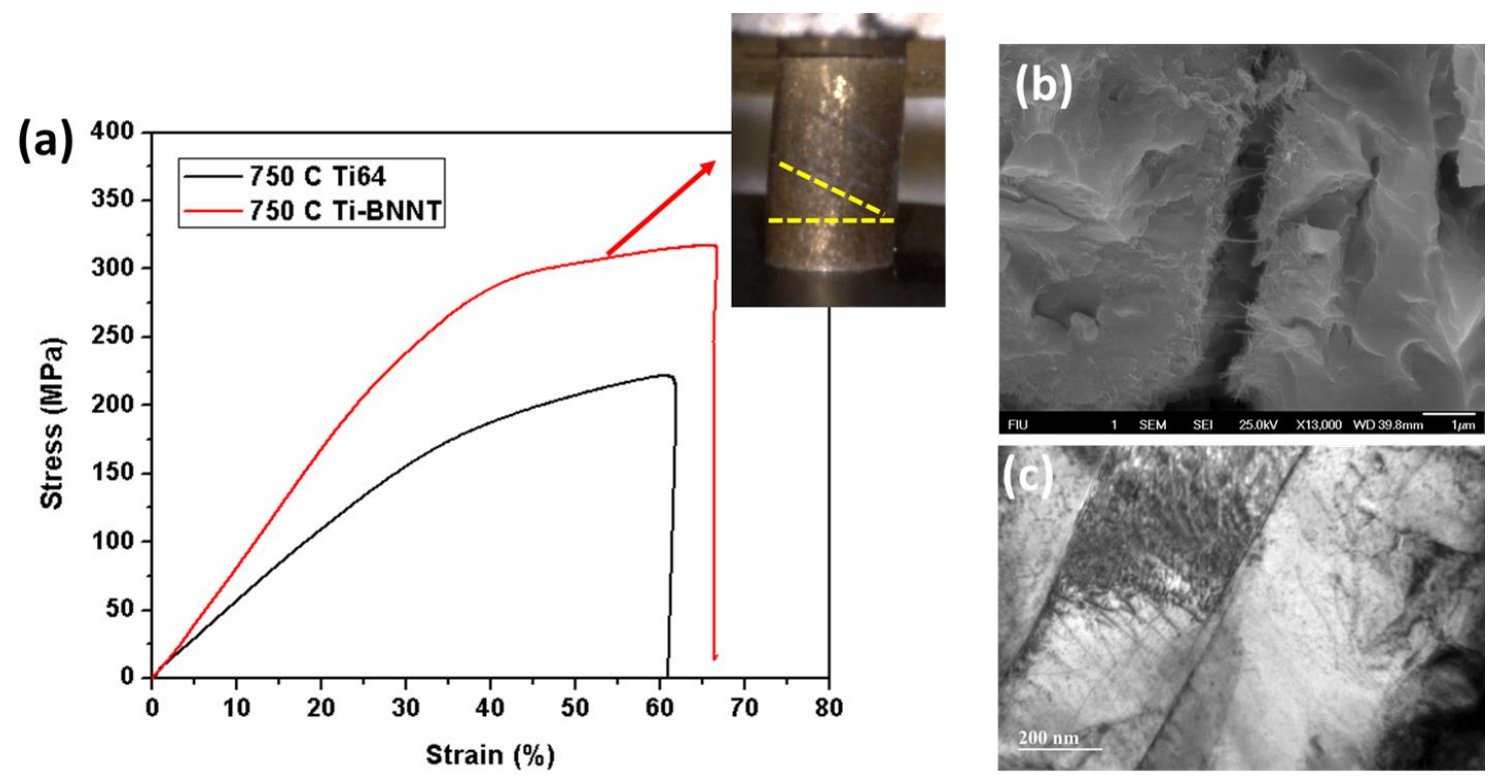

Figure 46 (a) Stress vs. Strain curve of Ti-BNNT and control (Ti64) specimens sintered at $750^{\circ} \mathrm{C}$. (b) the fracture surface of the composite evidencing crack-bridging by BNNTs as stress dissipation mechanism, (c) TEM micrograph showing the presence of shear band allocating dislocations and restricting their motion.

The ability of nanotubes to restrict plastic deformation in addition to the dense dislocation structure induced by high sintering pressures result in the reinforcement of the matrix. Strengthening of the matrix with the addition of BNNTs to the matrix results in compressive strengths of $292 \mathrm{MPa}$. Also, a closer look at the stress-strain curve of the composite as compared to the pure Ti6Al4V unravels the ability of the composite to bear stresses of up to $50 \%$ higher value than the pure Ti. Figure $46 \mathrm{~b}$ shows the fracture surface of the Ti-BNNT composite after experiencing a sudden fracture, where nanotubes are shown to interface the Ti-Ti particles and result bridging of the structure. Bridging is a 
common strengthening mechanism observed in composites reinforced by nanotubular structures. The extremely long morphology of nanotubes used in this study (up to $200 \mu \mathrm{m}$ ) is responsible for allowing to impart strong shearing forces restricting further deformations from occurring. Macroscale deformations undergone throughout the experimentation were captured via optical microscopy in real-time. A snapshot is shown in the inset of Figure 46a at a time point right before a catastrophic fracture of the Ti-BNNT composite occurs. Deformation of the composite is led by the initial formation of shear bands along a $20^{\circ}$ angle; increasing stresses are then responsible for causing fracture of the composite along with the shearing angles. The nature of the low temperature sintered Ti6Al4V evidenced the presence of shear bands representing the activation of slip planes along with the basal and prismatic directions. Where the presence of slip band boundaries act as barriers for dislocations by entrapment of the same (Figure 46c). Evaluating the fracture surface of the composites after experiencing catastrophic fracture during compression experiments is crucial to the understanding of deformation mechanisms governing the plastic deformation of the composite under stresses. The ability of the BNNTs to result in the reinforcement of the bulk structure was attested under compressive forces. The fracture surface of the composite is primarily governed by elongated dimpled features signaling the ductile shearing of the matrix (Figure 47). Ripple-like features can be observed at the surface along the direction of shearing (Figure 47a). Ripple features can be considered as barriers encountered during shear-dominated deformations. A closer look at the structures reveals the presence of nanotubular structures (BNNTs) at the grain boundaries (Figure 47c). In which, overcoming BNNT barriers to induce severe plastic deformation results in the pileup of plastically deformed Ti/BNNT interfaces. 

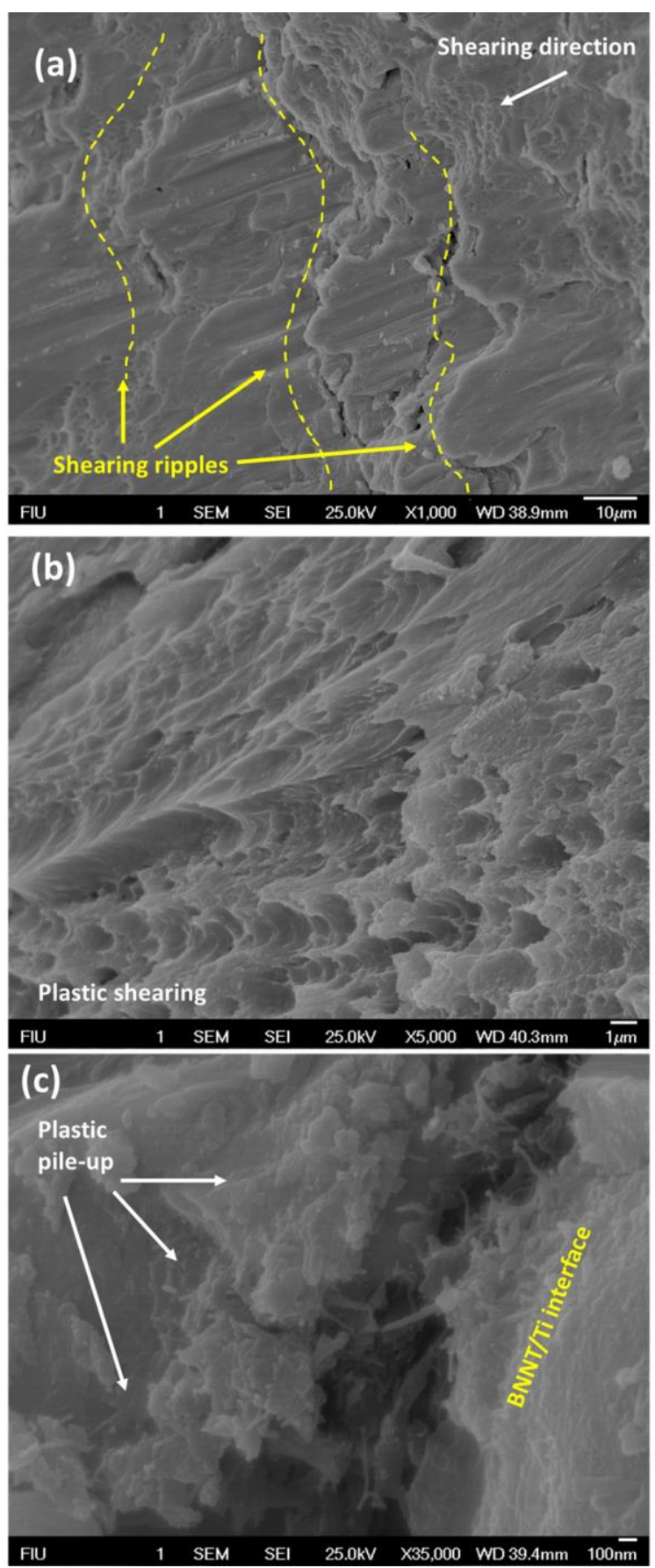

Figure 47 Fracture surface micrographs showing (a) the sheared failure surface of the composite with ripple-like features, (b) plastic shearing of $\mathrm{Ti}$ metal and (c) inducing plastic flow at the Ti/BNNT interfaces 
In a similar fashion Ti6AL4V and Ti-BNNT specimens sintered at $950^{\circ} \mathrm{C}$ and 60 MPa were subjected to axial compression loadings. The strong interfacial interactions observed in the evaluation above of the fracture surface of the composite will result in interesting energy and stress transfer mechanisms ${ }^{25,54}$. Figure 48 displays the characteristic stress vs. strain curve of Ti-BNNT composites and control specimen. Under increasing compressive load, the ductile nature of the Ti6Al4V specimen sintered at $950^{\circ} \mathrm{C}$ experiences buckling at approximately $40 \%$ strain. On the other hand, Ti-BNNT composites experience a catastrophic fracture at around $51.8 \%$ strain. The decreased fracture strain observed in the Ti-BNNT composite sintered at $750^{\circ} \mathrm{C}$ as compared to low temperature sintered specimens is attributed to the larger diffusion mechanisms experienced. Increasing temperatures result in the increase of diffusion coefficient of B $\left(\sim 2.62 \times 10^{-4} \mathrm{~m}^{2} / \mathrm{s}\right)$ to the Ti matrix. The diffusion of Boron atoms is known to occur via interstitials acting as an $\alpha$-phase stabilizer. Thus, resulting in the strengthening of the tougher and stiffer phase of Ti6Al4V. 

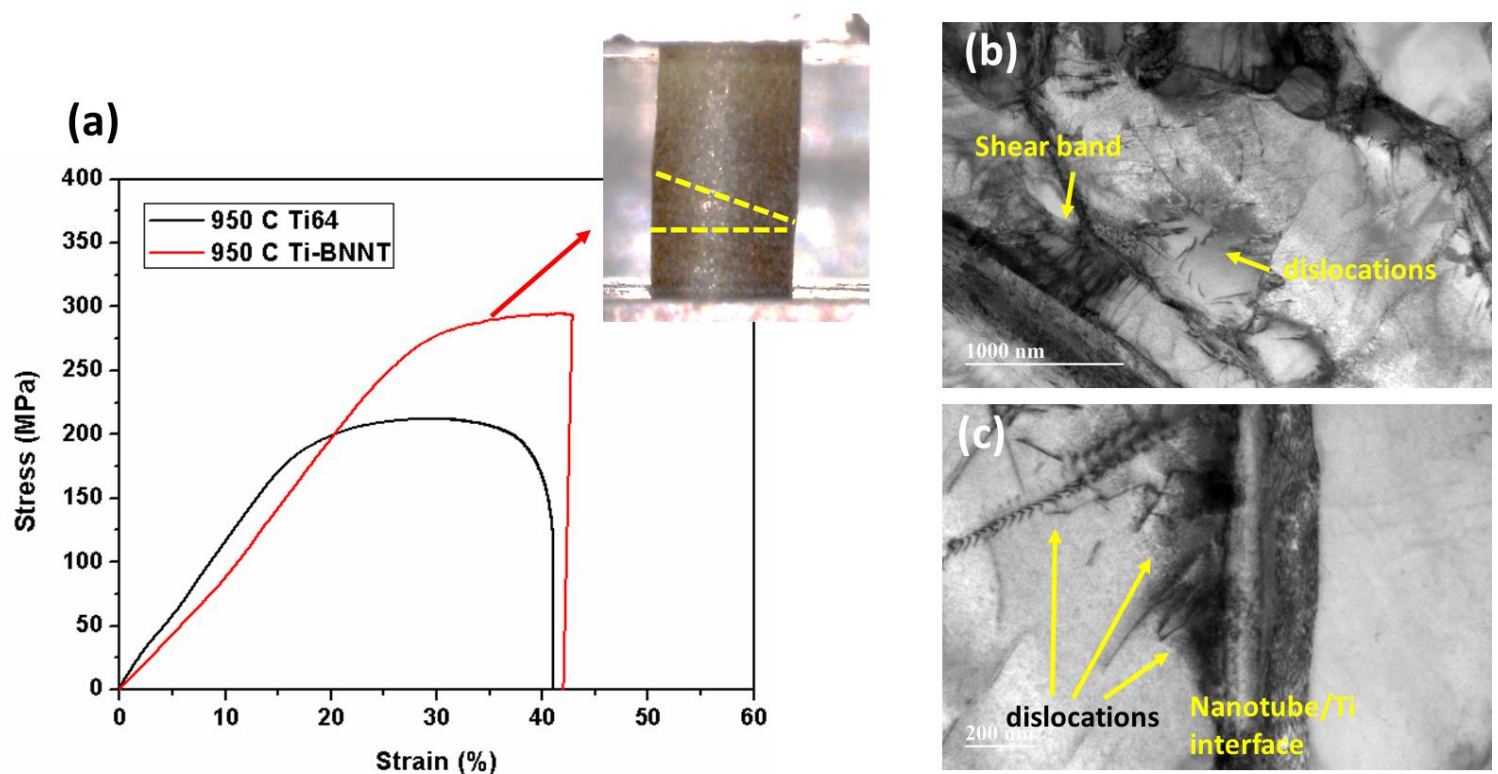

Figure 48 (a) Stress vs. Strain curve of Ti-BNNT and control (Ti64) specimens sintered at $9^{\circ} 0^{\circ} \mathrm{C}$. (b) and (c) TEM micrographs showing the presence of shear bands and dense dislocation microstructure with arrested dislocations at the interface of the BNNT and Ti

Plastic deformations experienced by the composite under compressive loads show yielding along a shear plane of $\sim 20-26^{\circ}$ as that observed in samples sintered at lower temperatures. The enhanced yield strength in the composite by almost $50 \%$ as compared to the pure Ti6Al4V sintered at similar conditions. The enhanced restriction to plastic deformation observed in the composite is attributed to the ability of nanotubes restricting dislocation movements. Figure $48 \mathrm{~b}$ and $\mathrm{c}$ show the presence of grown-in dislocations form by the collection of $\langle\mathrm{c}+\mathrm{a}\rangle$ dislocations and $\langle\mathrm{a}\rangle$ dislocations been restricted at the interface of the $\mathrm{Ti}$ matrix and the nanotube $\mathrm{105}^{106}$. The large presence of dislocations in the microstructure of the composite can be attributed to the activated slips induced by thermal stresses in the high temperature sintered samples. The elevated sintering temperatures of the specimens entails the phase transformation during cooling of secondary $\alpha$ grains within the transformed $\beta$-phases (attained during heating). The semi-coherency of interfaces 
between the $\alpha$ and $\beta$ phases gives rise to the dislocations driven by misfits between the two phases ${ }^{108}$. The increase of compressive stresses is then shown to result in the fracture of the composite resulting in compressive strengths of $282.3 \mathrm{MPa}$. It is important to highlight that the compressive strength of the composite sintered at higher temperatures is comparable to that achieved in low temperature sintered composites $\sim 292 \mathrm{MPa}$ and within the margin of error.

Further evaluation of the deformation mechanisms undergone by the Ti-BNNT composite under compressive forces was performed by evaluating the fracture surface shown in Figure 49. The fracture surface of the sintered specimens presents a mix mode fracture. The shearing of the metal matrix is observed in addition to dimple detachment from the surface. A closer look at sheared regions uncovers the presence of nanotubes been sheared along the direction of fracture Figure $49 \mathrm{c}$ and d. Thereby evidencing the strong interfacial interactions between the nanotubes and the titanium matrix. Regions where the pile-up is observed like that in Figure $49 \mathrm{~b}$ display nanotubes that have experienced deformations as a result of high localized compressive forces. The flexibility attained by the nanotubes as a result of its morphological attributes allows for bending of nanotubes to occur without experiencing a fracture of the nanotube. 

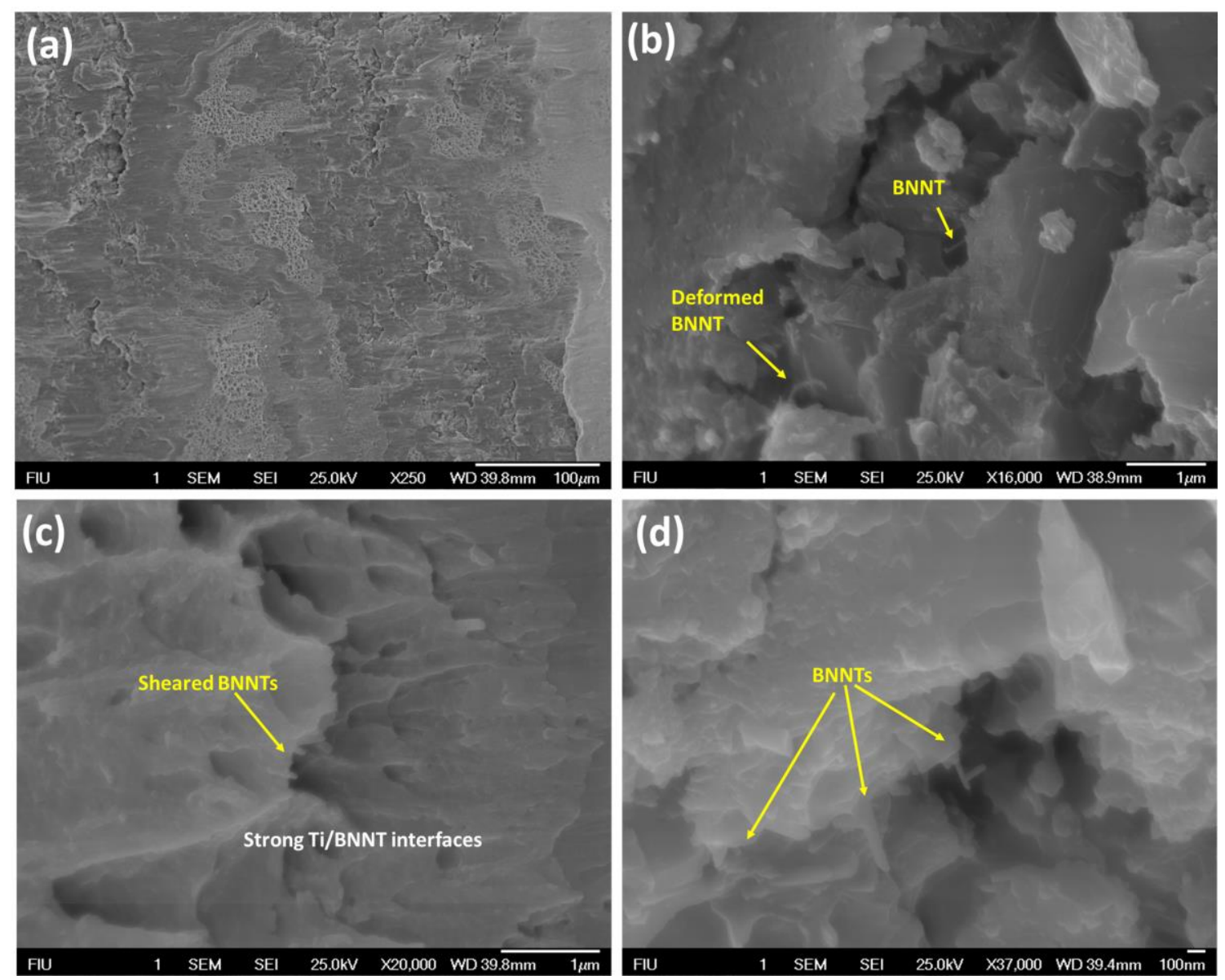

Figure 49 (a) Fracture surface of compressive failure experienced by the Ti-BNNT composite sintered at $9^{\circ 5} 0^{\circ} \mathrm{C}$, (b) BNNT/Ti interfaces with deformed BNNTs attesting the flexible nature of the nanotubes. (c) and (d) show the strong interfacial interactions between the nanotube and the Ti-matrix with sheared nanotubes.

\subsubsection{Nano- and Microscale strengthening of composites}

To gain an understanding of the microscopic deformation mechanisms and individual contributions of the BNNTs within the Ti-matrix, instrumented indentation at the nanoscale is explored. For which, the defect-free cross-sectional surface of each Ti-BNNT specimens sintered at low temperature and high-temperature sintering conditions were explored. Nanoindentation techniques have proven to be a powerful method for obtaining the mechanical characteristics of specimens with two or more phases ${ }^{4,53}$. Owing to the 
extremely small volumes interacting with the indenter, obtaining elastic modulus and nanohardness of specific phases is possible. The importance of the technique lies in the ability to obtain the contribution of the BNNTs to the reinforcement of the Ti-matrix as it was observed at a macro-scale in compression experiments. Figure 50 shows the characteristic load-displacement response of the Ti-BNNT samples sintered at different temperatures and pressures. The properties of the composites were extracted by indenting the surfaces with maximum loads of $8500 \mu \mathrm{N}$. A constant load hold was maintained at maximum load to allow sufficient time for creeping mechanisms not to influence the unloading portion of the curve.

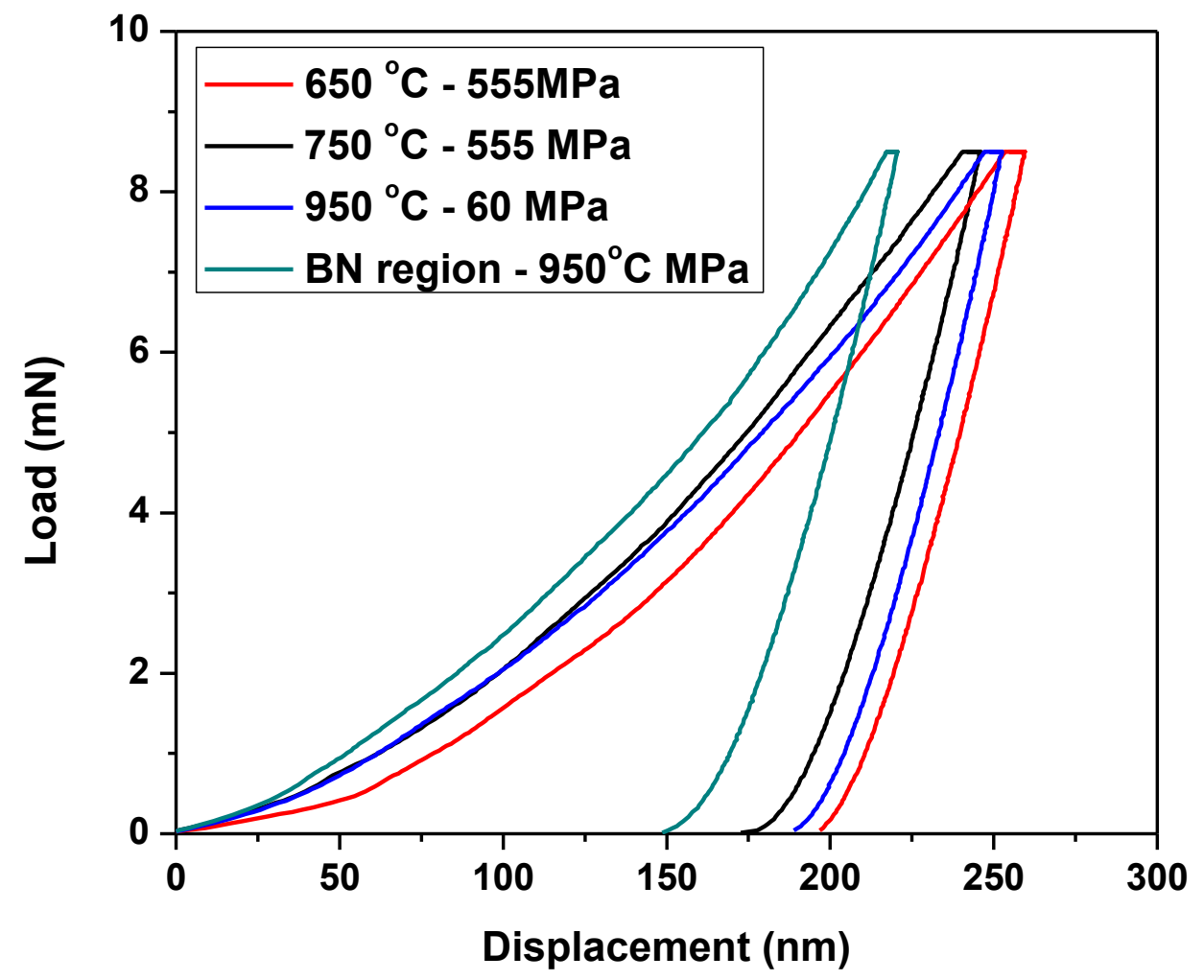

Figure 50 Load-displacement curves of Ti-BNNT composites as a function of sintering conditions.

A summary of the properties extracted for each composite samples and reference Ti6AL4V specimens is shown in Table 9. 
Table 9 Summary of mechanical properties obtained by nanoindentation technique

\begin{tabular}{|c|c|c|c|c|}
\hline Description & $\begin{array}{c}\text { Relative } \\
\text { Density (\%) }\end{array}$ & $\begin{array}{c}\text { Microhardness } \\
\text { (GPa) }\end{array}$ & $\begin{array}{c}\mathbf{E}_{\mathbf{r}} \\
(\mathbf{G P a})\end{array}$ & $\begin{array}{c}\text { Nanohardness } \\
\text { (GPa) }\end{array}$ \\
\hline $\begin{array}{c}\text { Ti64 } \\
650{ }^{\circ} \mathrm{C}-555 \mathrm{MPa}\end{array}$ & 98.9 & $3.7 \pm 0.04$ & $142.2 \pm 3.4$ & $4.75 \pm 0.3$ \\
\hline $\begin{array}{c}\text { Ti-BNNT } \\
650{ }^{\circ} \mathrm{C}-555 \mathrm{MPa}\end{array}$ & 98.3 & $3.6 \pm 0.08$ & $137.5 \pm 5.8$ & $5.27 \pm 0.3$ \\
\hline $\begin{array}{c}\text { Ti64 } \\
750{ }^{\circ} \mathrm{C}-555 \mathrm{MPa}\end{array}$ & 98.4 & $3.66 \pm 0.07$ & $141.1 \pm 8.5$ & $4.75 \pm 0.5$ \\
\hline $\begin{array}{c}\text { Ti-BNNT } \\
750{ }^{\circ} \mathrm{C}-555 \mathrm{MPa}\end{array}$ & 97.8 & $3.1 \pm 0.06$ & $132.4 \pm 8.7$ & $4.93 \pm 0.6$ \\
\hline $\begin{array}{c}\text { Ti64 } \\
950^{\circ} \mathrm{C}-60 \mathrm{MPa}\end{array}$ & 98.7 & $3.9 \pm 0.02$ & $142.5 \pm 8.0$ & $4.65 \pm 0.5$ \\
\hline $\begin{array}{c}\text { Ti-BNNT } \\
950^{\circ} \mathrm{C}-60 \mathrm{MPa}\end{array}$ & & (i): $4.8 \pm 0.5$ & $150.5 \pm 7.5$ & $5.6 \pm 0.5$ \\
\hline $\begin{array}{c}\mathrm{BN} \text { rich region } \\
950^{\circ} \mathrm{C}-60 \mathrm{MPa}\end{array}$ & & (ii): $3.5 \pm 0.2$ & $151.8 \pm 8.0$ & $7.22 \pm 0.8$ \\
\hline
\end{tabular}

It is evident from the curve that samples sintered at $650^{\circ} \mathrm{C}$ and $555 \mathrm{MPa}$ pressures, as a result of the poor densification and decreased bonding between the nanotubes and Timatrix result in maximum deformations of up to $250 \mathrm{~nm}$ and elastic modulus of $137.5 \pm$ 5.8 GPa. The slight decrease in elastic modulus of $\sim 3 \%$ as compared the pure Ti6Al4V sintered in similar conditions, accounts for the weak bonding interactions at the interface of the metallic matrix and nanotubes. The opposite is observed in comparing the nanohardness experienced with the addition of BNNT. In which, an $11 \%$ percent increase in nanohardness as compared to pure specimens sintered at $650^{\circ} \mathrm{C}$ characterize the ability of nanotubes to restrict dislocations and serving as additional barriers, even in the absence 
of ionic or covalent bonding. It is essential to highlight that the distinction of properties as a function of phases present is not captured in large scale indentation techniques such as that of microhardness. For which, a larger volume in contact is probed, and the effect of micro voids and microstructural defects have less impact on the mechanical characteristics of the composite. Thus, resulting in values similar to those of the pure Ti6Al4V, failing to capture poor sintering effects.

Increase in the sintering temperature to induce densification of the composite and overcome the BNNTs barrier for diffusion at temperatures of $750^{\circ} \mathrm{C}$ and pressures of 555 $\mathrm{MPa}$ was used. Elastic modulus was observed to decrease by $\sim 6 \%$ as compared to the control specimen sintered at similar conditions. In which a similar observation can be made as a function of weak bonding interaction between the nanotubes and the metal matrix. The slight increase in hardness observed by $\sim 4 \%$ in the composite can serve as evidence for the mechanism of reinforcement by which addition of BNNTs is governed.

Owing to the minimal chemical interactions resulting in ionic or covalent bonding at the Ti and nanotubes interface as it was observed by XRD, SEM and TEM analysis, strengthening of the composite is governed by the work of adhesion. In which, Van der Waal forces dictate the interactions between the nanotubes and the metal upon experiencing stresses. Strength at the interfaces is then a function of shear stresses $(\tau)$ and presents an inverse relationship with the radius of nanotubes $\left(R_{B N N T}\right):^{44}$

$$
\tau \alpha \frac{1}{R_{B N N T}}, A_{\text {effective }}
$$


Similarly, the relationship establishes a direct proportionality to the effective surface area ( $\left.A_{\text {effective }}\right)$ of the nanotube interacting with the matrix. The intrinsically small diameters of the nanotubes (up to $10 \mathrm{~nm}$ ) and extremely long (up to $200 \mu \mathrm{m}$ ) present and advantage to the interfacial strength of the composite. Where even in the absence of interfacial reaction phases, the strengthening of the composite can be deduced by the shearing interactions with the matrix.

Moreover, the mechanical characterization of high temperature sintered specimens was evaluated. The presence of interfacial reaction phases and partially reacted BNNTs was found to exhibit the largest increase in bulk compressive properties. Where the diffusion of Boron atoms into the $\mathrm{Ti}$ matrix to form $\mathrm{TiB}_{2}$ and $\mathrm{TiB}$ phases aid in the improvement of interfacial bonding ${ }^{48,54}$. The bimodal microstructural features observed in the composite were evaluated using elastic modulus and nanohardness in Figure 51. Region 1 is denominated as regions where evident agglomerates of nanotubes are not observed. These regions are primarily composed by $\alpha$ - and $\beta$ - phases with few nanotubes homogenously distributed along with the matrix. While, region 2 is considered as BN-rich regions, where the combination of reacted BNNTs and non-reacted BNNTs are present in larger volumes as compared to region 1 . Elastic modulus within region 1 resulted in elastic modulus improvement by $5 \%$ and regions 2 of about $7 \%$ as compared to the pure Ti64. 

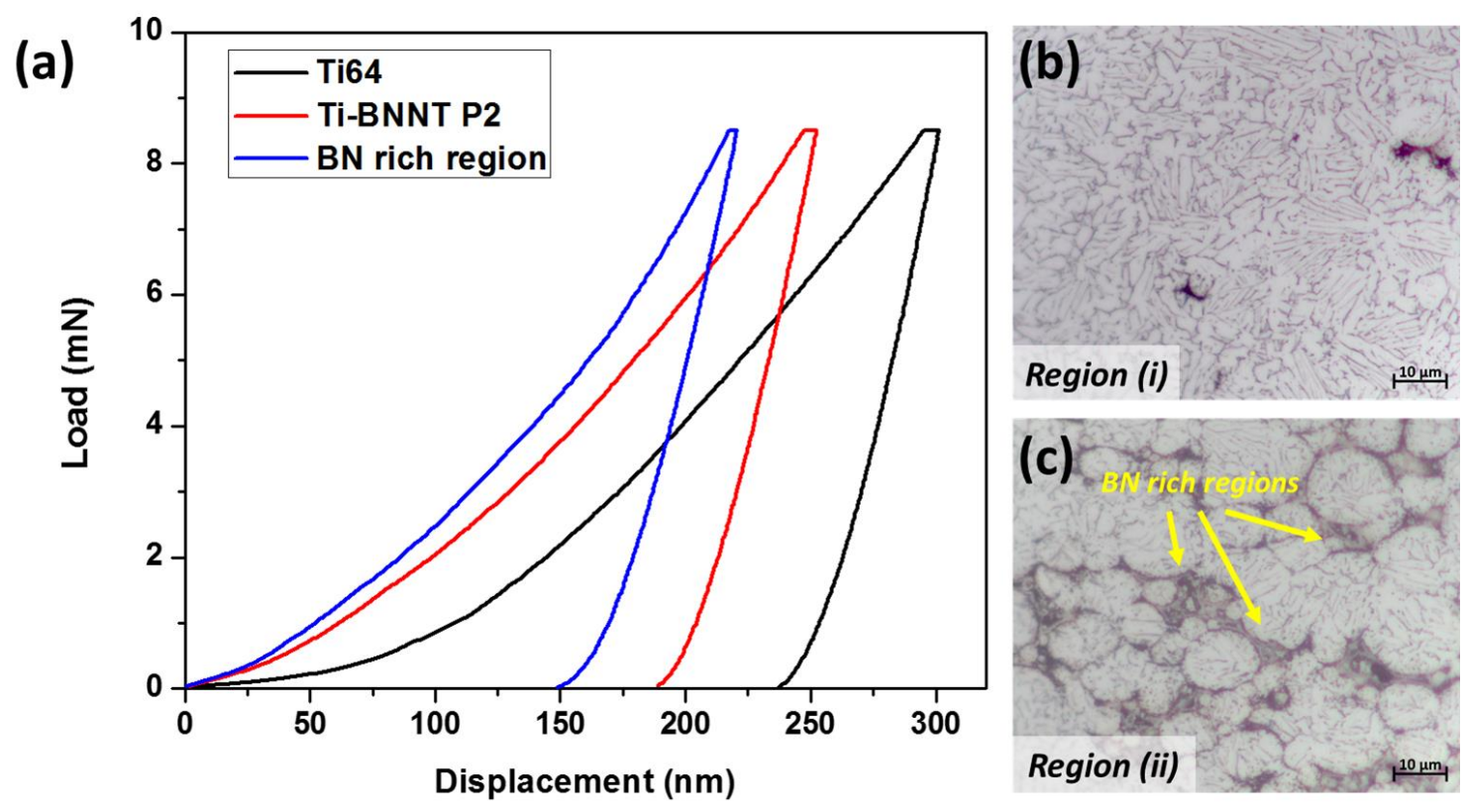

Figure 51 (a)Load-displacement curves of Ti-BNNT composite and Ti6Al4V control specimen showing the bimodal characteristics in the presence of (b) Ti dominant region and (c) $\mathrm{BN}$ rich region.

Although, small increments in elastic modulus are observed the predominantly higher values of nanohardness in region 2 of the Ti-BNNT composite of $7.22 \mathrm{GPa}$, signals the ability of nanotubes to restrict plastic deformations. At the nanoscale, their ability to pin dislocation from movement upon experiencing stresses. Thus, requiring much higher stresses to overcome barriers. The means by which stress transfer occurs from the ductile Ti-matrix to the stiff nanotubular reinforcements can be deduced by relating the energy required for fracture of the Ti matrix to happen and that required by the nanotube to dissociate from the matrix. Equation 12 describes the shear strength between the nanotube and the matrix by assuming the absence of chemical interactions between the nanotube and the Ti matrix. Upon experiencing high stresses, the chemically reacted BNNTs forming TiB phases will be responsible for the strengthening of the matrix. By which transfer of 
loads to the brittle $\mathrm{TiB}$ will occur via shearing at the interface of the nanotube/reaction phase and the metal matrix. Sun et al ${ }^{109}$. have reported the alignment of TiB phases along the direction highest shear stress upon experiencing compressive forces. Thus, bearing high stresses that are later transferred to the nanotubular $\mathrm{BN}^{109}$.

The mechanical reinforcement of Ti matrices by the addition of ultra-long and fine nanotubular structures has been shown across multiple length scales. Where the adhesion bonding predominantly by weal Van der Waals forces at the interface of nanotubes and Timatrix governed the strengthening observed in low temperature sintered composites. On the other hand, the controlled chemical reactions between the nanotube and the Ti-matrix attained after experiencing sintering at elevated temperatures resulted in strong interfacial interactions and strengthening of the matrix. The presence of reaction phases at the interface of the BNNT composites are considered as beneficial to the reinforcement of the matrix and crucial for reinforcing mechanisms to take place. 


\section{CHAPTER V: CONCLUSIONS}

In this study, Boron Nitride Nanotubes were introduced in the Titanium matrix to manufacture a composite with controlled interfacial reactions and improved mechanical properties. The sintering of Titanium at temperatures $\sim 50 \%$ less than those used in the conventional sintering is introduced for the first time to minimize interfacial reactions. The resulting morphological and mechanical evaluation was performed. The major findings of this study are summarized below:

$\checkmark$ Ultrasonication induced dispersion of BNNTs in acetone was proven to be an effective route for the mechanical disentanglement of BNNTs by inducing -OH functionalization and high energies introduced during sonication.

$\checkmark$ Surfactant-assisted BNNT dispersion was found not suitable due to entrapment of gases of volatile residual organics resulting in voids and residual Carbon impurities at high sintering temperature

$\checkmark$ Near full densification ( $98.9 \%$ ) of Ti6Al4V was achieved using low sintering temperatures of $650^{\circ} \mathrm{C}$ and high sintering pressures of $555 \mathrm{MPa}$.

- Morphological characteristics showed predominantly globular microstructures with the presence of a dense dislocation structure. Evidence of shear bands serving as entrapments for dislocation movement was also observed. This study proved that Ti could be sintered to a near full density at a much lower temperature.

$\checkmark$ Successful sintering of Ti-BNNT composites at low-temperature regimes at temperatures of $750^{\circ} \mathrm{C}$ and pressures of $555 \mathrm{MPa}$ was shown ( $\left.97.8 \%\right)$ :

- At the macroscale compressive yield strength was found to increase by $\sim 21 \%$. 
- Twin boundaries, crack bridging and BNNT pinning dislocations are found as mechanisms restricting the plastic deformation of the Ti-BNNT composite.

$\checkmark$ Successful sintering of Ti-BNNT composites at high sintering temperatures of $950^{\circ} \mathrm{C}$ with minimal interfacial reactions as a result of the kinetically restricted process was performed.

○ Bulk mechanical properties of the Ti- matrix are improved by up to $50 \%$ in its yield strength.

- At the nano-scale, a BN-rich region is identified with a high concentration of partially reacted BNNTs and non-reacted BNNTs with very high nanohardness of 7.22 $\mathrm{GPa}$ as compared to the pure Ti alloy which exhibits merely $4.65 \mathrm{GPa}$

- Strengthening of the composite is attributed to improved interfacial interactions by the formation of a thin layer of reacted phases which restrict dislocations movement. 


\section{CHAPTER VI: RECOMMENDATIONS \& FUTURE WORK}

This study proves the potential of BNNTs as a reinforcement to relatively high-temperature metals such as Titanium. The primary challenge of dispersing BNNTs with high surface energy was achieved via high energy tip sonication in acetone, which introduced free radicals that aids in their dispersion. Following recommendations are made for the future work, which could further the scientific understanding of Metal-BNNT composites.

\section{Dispersion of BNNTs}

$\checkmark$ Evaluating the effect of parameters in high energy ultrasonication (i.e. amplitude, time, and power) to the evolution of defects on the surface of BNNTs and quality of BNNT dispersion in aqueous medium.

$\checkmark$ Exploring the high energy ultrasonication induced dispersion of BNNTs with organic solvents and medium other than acetone could provide insights on the interaction of free radicals and potential functionalization of BNNTs.

\section{Low-Temperature Consolidation of Metal-BNNT composites}

$\checkmark$ The introduction of low-temperature sintering with high pressures could be extended to other metals such as $\mathrm{Al}$ and $\mathrm{Mg}$ to obtain BNNT reinforced composites. Low temperatures sintering as compared to conventional sintering represents a significant decrease in the energy and time required for the processing of metal-BNNT composites.

\section{Understanding Interfacial Behavior between Ti and BNNT at the atomic level}

$\checkmark$ High-resolution TEM evaluation to understand dislocation interactions with twin boundaries, which are characteristic of low temperature sintered Ti6Al4V and BNNTs to understand plastic deformation mechanisms undergone. 
$\checkmark$ X-ray photoelectron spectroscopy (XPS) analysis coupled with EDS to understand chemical interactions at Ti/BNNT interface and the nature of the bonds formed between Titanium and Boron to form $\mathrm{TiB}$ and $\mathrm{TiB}_{2}$. Such analysis will provide in-depth investigation of the degree of diffusion achieved by $\mathrm{B}$ and $\mathrm{N}$ atoms within the Ti-matrix to establish a correlation between phases contributing to the strengthening. 


\section{REFERENCES}

1. Rawal, S. P. Metal-matrix composites for space applications. JOM 53, 14-17 (2001).

2. Nautiyal, P., Gupta, A., Seal, S., Boesl, B. \& Agarwal, A. Reactive wetting and filling of boron nitride nanotubes by molten aluminum during equilibrium solidification. Acta Mater. 126, 124-131 (2017).

3. Chen, X., Dmuchowski, C. M., Park, C., Fay, C. C. \& Ke, C. Quantitative Characterization of Structural and Mechanical Properties of Boron Nitride Nanotubes in High Temperature Environments. Sci. Rep. 7, 11388 (2017).

4. Nautiyal, P. et al. Directionally Aligned Ultra-Long Boron Nitride Nanotube Induced Strengthening of Aluminum-Based Sandwich Composite. Adv. Eng. Mater. 18, 1747-1754 (2016).

5. Yamaguchi, M. et al. Utilization of multiwalled boron nitride nanotubes for the reinforcement of lightweight aluminum ribbons. Nanoscale Res. Lett. 8, 3 (2013).

6. Bhuiyan, M. M. H. et al. Boron nitride nanotube reinforced titanium metal matrix composites with excellent high-temperature performance. J. Mater. Res. 32, 3744 3752 (2017).

7. Kim, J. H., Pham, T. V., Hwang, J. H., Kim, C. S. \& Kim, M. J. Boron nitride nanotubes: synthesis and applications. Nano Converg. 5, 17 (2018).

8. Wang, J., Lee, C. H. \& Yap, Y. K. Recent advancements in boron nitride nanotubes. Nanoscale 2, 2028 (2010).

9. Davim, J. P. et al. Nanocomposites : Materials, Manufacturing and Engineering. (De Gruyter, 2013).

10. Brice, D. A. et al. Oxidation behavior and microstructural decomposition of Ti6Al-4V and Ti-6Al-4V-1B sheet. Corros. Sci. 112, 338-346 (2016).

11. Panda, K. B. \& Ravi Chandran, K. S. Synthesis of ductile titanium-titanium boride (Ti-TiB) composites with a beta-titanium matrix: The nature of TiB formation and composite properties. Metall. Mater. Trans. A 34, 1371-1385 (2003).

12. Gofrey, T. M. T., Goodwin, P. S. \& Ward-Close, C. M. Titanium Particulate Metal Matrix Composites - Reinforcement, Production Methods, and Mechanical Properties. Adv. Eng. Mater. 2, 85-91 (2000).

13. Chen, X. et al. Mechanical strength of boron nitride nanotube-polymer interfaces. Appl. Phys. Lett. 107, 253105 (2015). 
14. Zhi, C., Bando, Y., Tang, C. \& Golberg, D. Boron nitride nanotubes. Mater. Sci. Eng. R Reports 70, 92-111 (2010).

15. Chen, X. \& Ke, C. Structural and physical properties of boron nitride nanotubes and their applications in nanocomposites. in Boron Nitride Nanotubes in Nanomedicine 183-199 (William Andrew Publishing, 2016). doi:10.1016/B978-0323-38945-7.00012-2

16. Kim, K. S. et al. Hydrogen-Catalyzed, Pilot-Scale Production of Small-Diameter Boron Nitride Nanotubes and Their Macroscopic Assemblies. ACS Nano 8, 62116220 (2014).

17. Song, J., Liu, H. \& Shen, W. Dependence of electronic structures of multi-walled boron nitride nanotubes on layer numbers. Eur. Phys. J. D 72, 170 (2018).

18. Blase, X., Rubio, A., Louie, S. G. \& Cohen, M. L. Stability and Band Gap Constancy of Boron Nitride Nanotubes. Europhys. Lett. 28, 335-340 (1994).

19. Lauret, J. S. et al. Optical Transitions in Single-Wall Boron Nitride Nanotubes. Phys. Rev. Lett. 94, 1-4 (2005).

20. Bai, X. et al. Deformation-Driven Electrical Transport of Individual Boron Nitride Nanotubes. (2007). doi:10.1021/NL062540L

21. Kalay, S. et al. Synthesis of boron nitride nanotubes and their applications. Beilstein J. Nanotechnol. 6, 84-102 (2015).

22. Chopra, N. G. et al. Boron Nitride Nanotubes. Science (80-. ). 269, 966-967 (1995).

23. Kuznetsov, V. L., Mazov, I. N., Delidovich, A. I., Obraztsova, E. D. \& Loiseau, A. Thermodynamic analysis of nucleation of boron nitride nanotubes on metal particles. Phys. status solidi 244, 4165-4169 (2007).

24. Chen, Y., Fitz Gerald, J., Williams, J. S. \& Bulcock, S. Synthesis of boron nitride nanotubes at low temperatures using reactive ball milling. Chem. Phys. Lett. 299, 260-264 (1999).

25. Lahiri, D. et al. Insight into reactions and interface between boron nitride nanotube and aluminum. J. Mater. Res. 27, 2760-2770 (2012).

26. Oleg R. Lourie et al. CVD Growth of Boron Nitride Nanotubes. Chem. Mater. 12, 1808-1810 (2000).

27. Naumov, V. G. et al. Synthesis of Boron Nitride Multi-Walled Nanotubes by Laser Ablation Technique. Laser Phys. 19, 1198-1200 (2009). 
28. Smith, M. W. et al. Very long single- and few-walled boron nitride nanotubes via the pressurized vapor/condenser method. Nanotechnology 20, 505604 (2009).

29. Zhi, C. et al. Boron nitride nanotubes/polystyrene composites. J. Mater. Res. 21, 2794-2800 (2006).

30. Lahiri, D. et al. Boron nitride nanotube reinforced polylactide-polycaprolactone copolymer composite: Mechanical properties and cytocompatibility with osteoblasts and macrophages in vitro. Acta Biomater. 6, 3524-3533 (2010).

31. Chunyi Zhi et al. Purification of Boron Nitride Nanotubes through Polymer Wrapping. J. Phys. Chem. B 110, 1525-1528 (2006).

32. Yum, K. \& Yu, M.-F. Measurement of Wetting Properties of Individual Boron Nitride Nanotubes with the Wilhelmy Method Using a Nanotube-Based Force Sensor. Nano Lett. 6, 329-333 (2006).

33. Zhi, C. et al. Characteristics of Boron Nitride Nanotube-Polyaniline Composites. Angew. Chemie Int. Ed. 44, 7929-7932 (2005).

34. Kim, K. S. et al. Polymer nanocomposites from free-standing, macroscopic boron nitride nanotube assemblies. RSC Adv. 5, 41186-41192 (2015).

35. Jakubinek, M. B. et al. Thermal conductivity of bulk boron nitride nanotube sheets and their epoxy-impregnated composites. Phys. status solidi 213, 2237-2242 (2016).

36. Su, J., Xiao, Y. \& Ren, M. Enhanced thermal conductivity in epoxy nanocomposites with hybrid boron nitride nanotubes and nanosheets. Phys. status solidi 210, 2699-2705 (2013).

37. Yan, H., Tang, Y., Su, J. \& Yang, X. Enhanced thermal-mechanical properties of polymer composites with hybrid boron nitride nanofillers. Appl. Phys. A 114, 331337 (2014).

38. Zhi, C. et al. Towards Thermoconductive, Electrically Insulating Polymeric Composites with Boron Nitride Nanotubes as Fillers. Adv. Funct. Mater. 19, 18571862 (2009).

39. Kang, J. H. et al. Multifunctional Electroactive Nanocomposites Based on Piezoelectric Boron Nitride Nanotubes. ACS Nano 9, 11942-11950 (2015).

40. Terao, T. et al. Alignment of Boron Nitride Nanotubes in Polymeric Composite Films for Thermal Conductivity Improvement. J. Phys. Chem. C 114, 4340-4344 (2010). 
41. Huang, Q. et al. Enhancing superplasticity of engineering ceramics by introducing BN nanotubes. Nanotechnology 18, 485706 (2007).

42. Wang, W.-L. et al. Thermal Shock Resistance Behavior of Alumina Ceramics Incorporated with Boron Nitride Nanotubes. J. Am. Ceram. Soc. 94, 2304-2307 (2011).

43. Wang, W.-L. et al. Fabrication of Alumina Ceramic Reinforced with Boron Nitride Nanotubes with Improved Mechanical Properties. J. Am. Ceram. Soc. 94, 3636-3640 (2011).

44. Lahiri, D. et al. Boron nitride nanotube reinforced hydroxyapatite composite: Mechanical and tribological performance and in-vitro biocompatibility to osteoblasts. J. Mech. Behav. Biomed. Mater. 4, 44-56 (2011).

45. Bansal, N. P., Hurst, J. B. \& Choi, S. R. Boron Nitride Nanotubes-Reinforced Glass Composites. J. Am. Ceram. Soc. 89, 388-390 (2006).

46. Abass, M. A., Syed, A. A., Gervais, C. \& Singh, G. Synthesis and electrochemical performance of a polymer-derived silicon oxycarbide/boron nitride nanotube composite. RSC Adv. 7, 21576-21584 (2017).

47. Bhuiyan, M. M. H. et al. Boron nitride nanotube reinforced titanium metal matrix composites with excellent high-temperature performance. J. Mater. Res. 32, 3744 3752 (2017).

48. Bhuiyan, M. M. H., Li, L. H., Wang, J., Hodgson, P. \& Chen, Y. Interfacial reactions between titanium and boron nitride nanotubes. Scr. Mater. 127, 108-112 (2017).

49. Obraztsova, E. A. et al. Metal ion implantation of multiwalled boron nitride nanotubes. Scr. Mater. 67, 507-510 (2012).

50. Obraztsova, E. A. et al. Structural Changes of BN Nanotubes Irradiated by Al Ions. J. Nanoelectron. Optoelectron. 8, 87-90 (2013).

51. Yamaguchi, M. et al. Synthesis, structural analysis and in situ transmission electron microscopy mechanical tests on individual aluminum matrix/boron nitride nanotube nanohybrids. Acta Mater. 60, 6213-6222 (2012).

52. Yamaguchi, M., Meng, F., Firestein, K., Tsuchiya, K. \& Golberg, D. Powder metallurgy routes toward aluminum boron nitride nanotube composites, their morphologies, structures and mechanical properties. Mater. Sci. Eng. A 604, 9-17 (2014). 
53. Lahiri, D. et al. Boron nitride nanotubes reinforced aluminum composites prepared by spark plasma sintering: Microstructure, mechanical properties and deformation behavior. Mater. Sci. Eng. A 574, 149-156 (2013).

54. Nautiyal, P., Zhang, C., Boesl, B. \& Agarwal, A. Non-equilibrium wetting and capture of boron nitride nanotubes in molten aluminum during plasma spray. Scr. Mater. 151, 71-75 (2018).

55. Nautiyal, P., Gupta, A., Seal, S., Boesl, B. \& Agarwal, A. Reactive wetting and filling of boron nitride nanotubes by molten aluminum during equilibrium solidification. Acta Mater. 126, 124-131 (2017).

56. Rohmann, C. et al. Interaction of Boron Nitride Nanotubes with Aluminum: A Computational Study. J. Phys. Chem. C 122, 15226-15240 (2018).

57. Bisht, A. et al. Effect of warm rolling and annealing on the mechanical properties of aluminum composite reinforced with boron nitride nanotubes. Mater. Sci. Eng. A 710, 366-373 (2018).

58. Antillon, M., Nautiyal, P., Loganathan, A., Boesl, B. \& Agarwal, A. Strengthening in Boron Nitride Nanotube Reinforced Aluminum Composites Prepared by Roll Bonding. Adv. Eng. Mater. 20, 1800122 (2018).

59. Sieniawski, J., Ziaja, W., Kubiak, K. \& Motyka, M. Microstructure and Mechanical Properties of High Strength Two-Phase Titanium Alloys. (2013). doi: $10.5772 / 56197$

60. Kaur, M. \& Singh, K. Review on titanium and titanium based alloys as biomaterials for orthopaedic applications. Mater. Sci. Eng. C 102, 844-862 (2019).

61. Ramesh, A. \& Banoth, S. Influence of Thermo Mechanical Properties Parameters on Titanium Metal Matrix Composite and Ti-6Al-4V for Aerospace Applications. Int. Conf. Inf. Eng. Manag. Secur. 1, (2014).

62. Ravi Chandran, K. S., Panda, K. B. \& Sahay, S. S. TiBw-reinforced Ti composites: Processing, properties, application prospects, and research needs. JOM 56, 42-48 (2004).

63. Azarniya, A. et al. Metallurgical Challenges in Carbon Nanotube-Reinforced Metal Matrix Nanocomposites. Metals (Basel). 7, 384 (2017).

64. Kondoh, K., Threrujirapapong, T., Umeda, J. \& Fugetsu, B. High-temperature properties of extruded titanium composites fabricated from carbon nanotubes coated titanium powder by spark plasma sintering and hot extrusion. Compos. Sci. Technol. 72, 1291-1297 (2012). 
65. Rohmann, C., Sun, Q. \& Searles, D. J. Interaction of Al, Ti, and Cu Atoms with Boron Nitride Nanotubes: A Computational Investigation. J. Phys. Chem. C 120, 3509-3518 (2016).

66. Aich, S. \& Ravi Chandran, K. S. TiB whisker coating on titanium surfaces by solid-state diffusion: Synthesis, microstructure, and mechanical properties. Metall. Mater. Trans. A 33, 3489-3498 (2002).

67. Xue, Y. et al. Aluminum matrix composites reinforced with multi-walled boron nitride nanotubes fabricated by a high-pressure torsion technique. Mater. Des. 88, 451-460 (2015).

68. Lahiri, D. \& Agarwal, A. Boron Nitride Nanotubes as Nanofillers/Reinforcement for Polymer, Ceramic, and Metal Matrix Composites. in Nanotubes and Nanosheets 495-524 (CRC Press, 2015). doi:10.1201/b18073-24

69. Racles, C., Iacob, M., Butnaru, M., Sacarescu, L. \& Cazacu, M. Aqueous dispersion of metal oxide nanoparticles, using siloxane surfactants. Colloids Surfaces A Physicochem. Eng. Asp. 448, 160-168 (2014).

70. Tsai, W.-B., Kao, J.-Y., Wu, T.-M. \& Cheng, W.-T. Dispersion of Titanium Oxide Nanoparticles in Aqueous Solution with Anionic Stabilizer via Ultrasonic Wave. $J$. Nanoparticles 2016, 1-9 (2016).

71. Varghese, S. Role of surfactants on the stability of nano-zinc oxide dispersions. Part. Sci. Technol. 35, 67-70 (2017).

72. Sesis, A. et al. Influence of Acoustic Cavitation on the Controlled Ultrasonic Dispersion of Carbon Nanotubes. J. Phys. Chem. B 117, 15141-15150 (2013).

73. Cheng, Q., Debnath, S., Gregan, E. \& Byrne, H. J. Ultrasound-Assisted SWNTs Dispersion: Effects of Sonication Parameters and Solvent Properties. J. Phys. Chem. C 114, 8821-8827 (2010).

74. Kharissova, O. V., Kharisov, B. I. \& de Casas Ortiz, E. G. Dispersion of carbon nanotubes in water and non-aqueous solvents. RSC Adv. 3, 24812 (2013).

75. BNNT LLC. Boron Nitride Nanotubes. (2014).

76. Smith McWilliams, A. D. et al. Surfactant-assisted individualization and dispersion of boron nitride nanotubes. Nanoscale Adv. 1, 1096-1103 (2019).

77. Lin, Y. et al. Aqueous Dispersions of Few-Layered and Monolayered Hexagonal Boron Nitride Nanosheets from Sonication-Assisted Hydrolysis: Critical Role of Water. J. Phys. Chem. C 115, 2679-2685 (2011). 
78. Kim, D. et al. Sonication-assisted alcoholysis of boron nitride nanotubes for their sidewalls chemical peeling. Chem. Commun. 51, 7104-7107 (2015).

79. Ganji, M. D. \& Rezvani, M. Boron nitride nanotube based nanosensor for acetone adsorption: a DFT simulation. J. Mol. Model. 19, 1259-1265 (2013).

80. Tam, D. K. Y., Ruan, S., Gao, P. \& Yu, T. High-performance ballistic protection using polymer nanocomposites. Adv. Mil. Text. Pers. Equip. 213-237 (2012). doi:10.1533/9780857095572.2.213

81. Zare, Y., Rhee, K. Y. \& Hui, D. Influences of nanoparticles aggregation/agglomeration on the interfacial/interphase and tensile properties of nanocomposites. Compos. Part B Eng. 122, 41-46 (2017).

82. Yu, J., Chen, Y. \& Cheng, B. M. Dispersion of boron nitride nanotubes in aqueous solution with the help of ionic surfactants. Solid State Commun. 149, 763-766 (2009).

83. Drioli, E., Criscuoli, A. \& Curcio, E. Membrane materials. in Membrane contactors : fundamentals, applications and potentialities 40-104 (Elsevier, 2006).

84. Lin, Y. \& Alexandridis, P. Temperature-Dependent Adsorption of Pluronic F127 Block Copolymers onto Carbon Black Particles Dispersed in Aqueous Media. $J$. Phys. Chem. B 106, 10834-10844 (2002).

85. Gao, Q. et al. Synthesis and characterization of novel amphiphilic copolymer stearic acid-coupled F127 nanoparticles for nano-technology based drug delivery system. Colloids Surfaces B Biointerfaces 88, 741-748 (2011).

86. Bohorquez, M., Koch, C., Trygstad, T. \& Pandit, N. A Study of the TemperatureDependent Micellization of Pluronic F127. J. Colloid Interface Sci. 216, 34-40 (1999).

87. Jebari, M. M., Ghaouar, N., Aschi, A. \& Gharbi, A. Aggregation behaviour of Pluronic ${ }^{\circledR}$ L64 surfactant at various temperatures and concentrations examined by dynamic light scattering and viscosity measurements. Polym. Int. 55, 176-183 (2006).

88. Miljevic, B. et al. To Sonicate or Not to Sonicate PM Filters: Reactive Oxygen Species Generation Upon Ultrasonic Irradiation. Aerosol Sci. Technol. 48, 12761284 (2014).

89. Niemczewski, B. A comparison of ultrasonic cavitation intensity in liquids. Ultrasonics 18, 107-110 (1980). 
90. Ejaz, M. et al. Surface-initiated atom transfer radical polymerization of glycidyl methacrylate and styrene from boron nitride nanotubes. J. Mater. Chem. C 00, 1-3 (2013).

91. Sainsbury, T. et al. Oxygen Radical Functionalization of Boron Nitride Nanosheets. J. Am. Chem. Soc. 134, 18758-18771 (2012).

92. Weng, Q., Wang, X., Wang, X., Bando, Y. \& Golberg, D. Functionalized hexagonal boron nitride nanomaterials: emerging properties and applications. Chem. Soc. Rev. 45, 3989-4012 (2016).

93. Lin, S., Ashrafi, B., Laqua, K., Su Kim, K. \& Simard, B. Covalent derivatization of boron nitride nanotubes with peroxides and their application in polycarbonate composites. New J. Chem. 41, 7571-7577 (2017).

94. Shahedi Asl, M., Sabahi Namini, A., Motallebzadeh, A. \& Azadbeh, M. Effects of sintering temperature on microstructure and mechanical properties of spark plasma sintered titanium. Mater. Chem. Phys. 203, 266-273 (2018).

95. Xu, L., Guo, R., Bai, C., Lei, J. \& Yang, R. Effect of Hot Isostatic Pressing Conditions and Cooling Rate on Microstructure and Properties of Ti-6Al-4V Alloy from Atomized Powder. J. Mater. Sci. Technol. 30, 1289-1295 (2014).

96. Adegbenjo, A. O., Obadele, B. A. \& Olubambi, P. A. Densification, hardness and tribological characteristics of MWCNTs reinforced Ti6Al4V compacts consolidated by spark plasma sintering. J. Alloys Compd. 749, 818-833 (2018).

97. Crosby, K. et al. Enhancement in Ti-6Al-4V sintering via nanostructured powder and spark plasma sintering. Powder Metall. 57, 147-154 (2014).

98. Li, A. et al. Microstructure and mechanical properties of Y2O3 reinforced Ti6Al4V composites fabricated by spark plasma sintering. J. Alloys Compd. 768, 49-56 (2018).

99. Wu, Y. et al. An Integrated Forming Process for Manufacturing Ti6A14V Impeller with a Functional Coating Layer Using Hot Isostatic Pressing. Mater. Manuf. Process. 30, 756-765 (2015).

100. Singh, D., Pandey, P. M. \& Kalyanasundaram, D. Optimization of pressure-less microwave sintering of Ti6Al4V by response surface methodology. Mater. Manuf. Process. 33, 1835-1844 (2018).

101. Azevedo, C. R. F., Rodrigues, D. \& Beneduce Neto, F. Ti-Al-V powder metallurgy (PM) via the hydrogenation-dehydrogenation (HDH) process. J. Alloys Compd. 353, 217-227 (2003). 
102. Diouf, S. \& Molinari, A. Densification mechanisms in spark plasma sintering: Effect of particle size and pressure. Powder Technol. 221, 220-227 (2012).

103. Khorsand Zak, A., Abd. Majid, W. H., Abrishami, M. E. \& Yousefi, R. X-ray analysis of $\mathrm{ZnO}$ nanoparticles by Williamson-Hall and size-strain plot methods. Solid State Sci. 13, 251-256 (2011).

104. Hansen, N. \& Barlow, C. Y. Plastic Deformation of Metals and Alloys. Phys. Metall. 1681-1764 (2014). doi:10.1016/B978-0-444-53770-6.00017-4

105. Wang, Q. \& Liu, Z. Plastic deformation induced nano-scale twins in Ti-6Al-4V machined surface with high speed machining. Mater. Sci. Eng. A 675, 271-279 (2016).

106. Nervo, L., King, A., Fitzner, A., Ludwig, W. \& Preuss, M. A study of deformation twinning in a titanium alloy by X-ray diffraction contrast tomography. Acta Mater. 105, 417-428 (2016).

107. Peterson, N. L. Diffusion in Refractory Metals. (1961).

108. Balachandran, S., Kumar, S. \& Banerjee, D. On recrystallization of the $\alpha$ and $\beta$ phases in titanium alloys. Acta Mater. 131, 423-434 (2017).

109. Sun, S. \& Lu, W. Effect of Hybrid Reinforcements on the Microstructure and Mechanical Properties of Ti-5Al-5Mo-5V-Fe-Cr Titanium Alloy. Metals (Basel). 7, 250 (2017). 